EPJ Web of Conferences 22, 00006 (2012)

DOI: $10.1051 /$ epjconf/20122200006

(C) Owned by the authors, published by EDP Sciences, 2012

\title{
Crystallography: Symmetry groups and group representations
}

\author{
B. Grenier ${ }^{1}$ and R. Ballou ${ }^{2}$ \\ 1 SPSMS, UMR-E 9001, CEA-INAC / UJF-Grenoble, MDN, 38054 Grenoble, France \\ 2 Institut Néel, CNRS / UJF, 25 rue des Martyrs, BP. 166, 38042 Grenoble Cedex 9, France
}

\begin{abstract}
This lecture is aimed at giving a sufficient background on crystallography, as a reminder to ease the reading of the forthcoming chapters. It more precisely recalls the crystallographic restrictions on the space isometries, enumerates the point groups and the crystal lattices consistent with these, examines the structure of the space group, which gathers all the spatial invariances of a crystal, and describes a few dual notions. It next attempts to familiarize us with the representation analysis of physical states and excitations of crystals.
\end{abstract}

\section{INTRODUCTION}

Crystallography covers a wide spectrum of investigations: $i-$ it aspires to get an insight into crystallization phenomena and develops methods of crystal growths, which generally pertains to the physics of non linear irreversible processes; ii- it geometrically describes the natural shapes and the internal structures of the crystals, which is carried out most conveniently by borrowing mathematical tools from group theory; iii- it investigates the crystallized matter at the atomic scale by means of diffraction techniques using X-rays, electrons or neutrons, which are interpreted in the dual context of the reciprocal space and transposition therein of the crystal symmetries; iv- it analyzes the imperfections of the crystals, often directly visualized in scanning electron, tunneling or force microscopies, which in some instances find a meaning by handling unfamiliar concepts from homotopy theory; v- it aims at providing means for discerning the influences of the crystal structure on the physical properties of the materials, which requires to make use of mathematical methods from representation theory. A number of extensions of this standard crystallography in addition were formulated, for instance towards euclidean spaces of dimension $v>3$, towards structures that are periodic solely in sub-spaces of dimension $\mu<v$, towards combination of geometric symmetry with changes of a property, giving rise to color groups and other alike, towards modulated and composite incommensurate crystals, answerable to the formalism of the superspace groups, towards quasi-crystals, apprehended by means of irrational cuts and projections of $v$-dimensional crystals, or else towards more general multi-metrical not necessarily euclidean crystallography [1,2]. A small part only of these topics, to be precise the geometrical description and the representation analysis of the symmetry groups of the infinite perfect 3-dimensional crystals, will be addressed hereafter. We shall resort to only elementary aspects of the concepts discussed in the preceding two chapters, by Canals and Schober and by Ballou.

\section{SYMMETRY GROUPS}

The word crystal stems from Greek "krustallas" and means "solidified by the cold". More precisely it designates an ordered state of matter with periodic arrangements of ions, atoms or molecules. Although this formally distinguishes the crystal state from the other states of solid matter, for instance glasses, gels

This is an Open Access article distributed under the terms of the Creative Commons Attribution-Noncommercial License 3.0, which permits unrestricted use, distribution, and reproduction in any noncommercial medium, provided the original work is properly cited. 


\section{EPJ Web of Conferences}

or else foams, it is not always straightforward to assert that a real material is crystalline. All depends on the spatial extension over which the correlations in atomic positions are examined and thereby on the experimental means to probe the crystallinity. It implicitly will be assumed that the coherence length is infinite. This amounts to consider solely ideal crystals without defects or even a surface, which is an excellent approximation in most instances. The ideal crystal is characterized by two basic symmetries, orientation symmetry and translation symmetry, and by their combinations. Orientation symmetry comes out in the anisotropy of the physical properties of the crystals. It reveals itself in macroscopic physics, most spectacularly in the external shape of crystals, which often present natural faces, and in mechanical properties, optical properties, electric conductivity, .... Translation symmetry accounts for the periodicity of the physical properties of the crystals. It is responsible for the phenomenon of diffraction and gives rise to specific dynamics, phonons (lattice vibrations), magnons (spin waves), ..., with coherent propagations. These basic symmetries are mutually constrained. Section 2.1 will describe the orientation symmetries, recalling the restrictions inherent to the discretized translations, then will enumerate the 32 crystallographic point groups that can be built from them and discuss the method of stereographic projections to geometrically represent these groups. Section 2.2 will be concerned with the translation symmetries and will enumerate the 6 conventional cells, the 7 crystal systems and the 14 Bravais lattices consistent with the orientation symmetries. The notions of rows and net planes, especially useful for diffraction, will be briefly recalled. Section 2.3 will describe glide planes and screw axes, completing the inventory of the crystal symmetries, then will discuss the space groups, gathering all the isometries for a given crystal. The way in which the relevant data on a space group are transcribed in the International Tables For Crystallography (ITC) [1] will be explained, from two selected examples. The international (Hermann-Mauguin) symbols will be used for both the point groups and the space groups, since these are the most commonly encountered in crystallography and are much more convenient for the space groups. The Schoenflies notation nevertheless will be considered for the point groups, since quite often this is used in spectroscopic investigations. Section 2.4 will succinctly call back the dual notions of the reciprocal space and Brillouin zone, which are essential when experimentally determining the crystal structure or investigating propagating excitations, by means of $\mathrm{X}$-rays or neutrons scattering techniques.

\subsection{Orientation symmetry}

An orientation symmetry in the $v$-dimensional space $\mathcal{E}_{v}$ designates an euclidean isometry that leaves at least one point fixed. It thus is called point symmetry as well. Choosing the fixed point as the origin $\mathrm{O}$ in $\mathcal{E}_{v}$ and selecting an orthogonal frame $\mathrm{O} x_{1} \cdots x_{v}$, it is put in bijective correspondence with a $v$-by- $v$ real orthogonal matrix, namely a matrix $\alpha$ with real entries such that $\alpha^{T} \alpha=\imath_{v}$ where $\alpha^{T}$ symbolizes the transpose of $\alpha$ and $l_{v}$ the $\nu$-by- $\nu$ unit matrix. It follows that the determinant of $\alpha$ necessarily is $\operatorname{Det}(\alpha)= \pm 1$, since $\operatorname{Det}\left(\alpha^{T}\right)=\operatorname{Det}(\alpha)$ and $\operatorname{Det}(\alpha \beta)=\operatorname{Det}(\alpha) \operatorname{Det}(\beta) \forall \alpha \forall \beta$. The orientation symmetry is termed proper if $\operatorname{Det}(\alpha)=+1$ and improper otherwise. A real orthogonal matrix $\alpha$ is diagonalizable in the field $\mathbb{R}$ of real numbers if and only if it is an involution, namely if and only if $\alpha^{2}=l_{v}$. Its eigenvalues then are +1 with multiplicity $p$ and -1 with multiplicity $q=v-p$. Intuitively, this describes a reflection about the $p$-dimensional subspace $\mathcal{E}_{p}^{+}=\left\{\mathrm{M} \in \mathcal{E}_{v} \mid \overrightarrow{\mathrm{OM}} \in \operatorname{ker}\left(\alpha-l_{\nu}\right)\right\}$ of the fixed points of the involution in $\mathcal{E}_{v}$. $\operatorname{ker}\left(\alpha-\imath_{\nu}\right)$ is the set of vectors $\vec{v}$ such that $\left(\alpha-\imath_{\nu}\right) \vec{v}=\overrightarrow{0}$. There exists only one reflection about the singleton $\mathcal{E}_{0}^{+}=\{\mathrm{O}\}$, called inversion through the origin. This is a proper symmetry for even $v$ and an improper symmetry for odd $v$. We get a hyperplane reflection for $p=v-1$ and a flip about $\mathcal{E}_{p}^{+}$for $p=v-2$. According to the Cartan-Dieudonné theorem, every orientation symmetry is the composition of at minimum $\mu=v-\operatorname{dim}\left[\operatorname{ker}\left(\alpha-\imath_{v}\right)\right]$ reflections. When $v \geq 3$, any composition of two hyperplane reflections is equivalent to the composition of two flips. Within our everyday space, namely for $v=3$, a hyperplane reflection is a mirror $(p=2)$ and a flip about an axis a $\pi$-rotation $(p=1)$. 

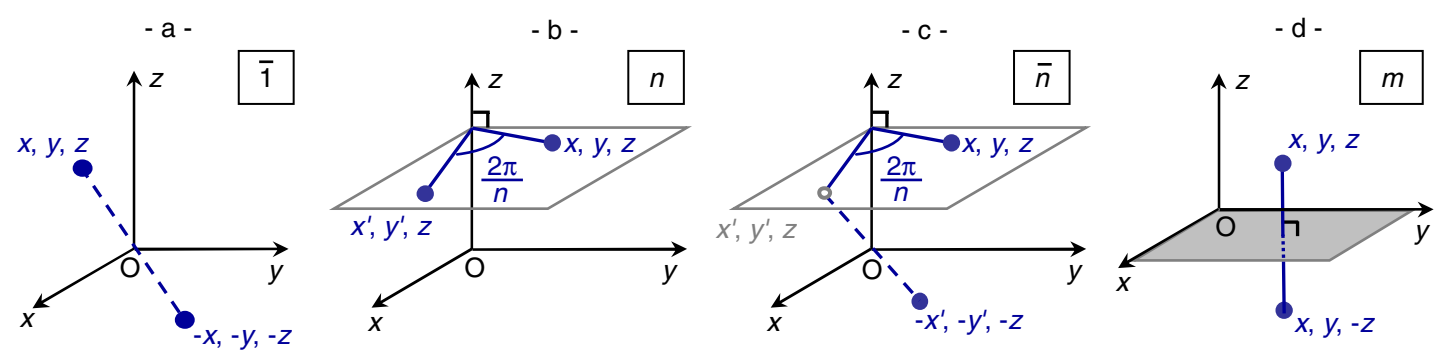

Figure 1. The basic elementary point symmetries in a crystal and their associated point group symbol. a- inversion through the origin (fixed point), symbol: $\overline{1}, \mathrm{~b}$ - rotation of order $n$ about the $z$-axis (fixed line), symbol: $n$ (this is a rotation of angle $2 \pi / n$ ), c- rotoinversion about the $z$-axis (fixed point), symbol: $\bar{n}$ (this is a rotation of angle $2 \pi / n$ followed by an inversion - the empty circle stands for an intermediate immaterial position), d- reflection about a plane or mirror (fixed plane), symbol: $m$ (it may be emphasized that this is in effect the rotoinversion $\overline{2}$, but the symbol $m$ is preferentially used).

Addressing more specifically the 3-dimensional crystallography, it is customary to distinguish as basic elementary orientation symmetries, the inversion $\mathcal{I}$ through the origin, the rotation $\mathcal{R}(\hat{u}, \varphi)$ by an angle $\varphi$ about a $u$-axis of unit vector $\hat{u}$, the rotoinversion $\mathcal{I} \circ \mathcal{R}(\hat{u}, \varphi)=\mathcal{R}(\hat{u}, \varphi) \circ \mathcal{I}$ and the mirror $\mathcal{M}_{\hat{u}}=\mathcal{I} \circ \mathcal{R}(\hat{u}, \pi)$, as sketched in figure 1. Use also is made, generally in spectroscopy, of rotoreflections $\mathcal{M}_{\hat{u}} \circ \mathcal{R}(\hat{u}, \varphi)=\mathcal{R}(\hat{u}, \varphi) \circ \mathcal{M}_{\hat{u}}$, which easily transpose to rotoinversions as $\mathcal{M}_{\hat{u}} \circ$ $\mathcal{R}(\hat{u}, \varphi)=\mathcal{I} \circ \mathcal{R}(\hat{u}, \pi) \circ \mathcal{R}(\hat{u}, \varphi)=\mathcal{I} \circ \mathcal{R}(\hat{u}, \varphi+\pi)$. As a matter of fact, only a few discrete rotation angles of the form $\varphi=2 \pi / n$ are allowed in the crystals (as will be seen in subsection 2.1.1), where $n$ is an integer called the order of the rotation. On the other hand, when a crystal is invariant under a given symmetry it also is invariant under the repeated applications of this symmetry. Thus, in figure 1 only the first application of the considered orientation symmetry is represented, but this could have been applied $n$ times until getting back to the starting point. A rotation of order 4 for example produces 4 different positions, by applying it a first, a second, a third then a fourth time, this last composition leading to the identity since $4 \times 2 \pi / 4=2 \pi$. The international convention is to symbolize a crystal rotation, its axis and the cyclic group it generates, all by its order $n$. A crystal rotoinversion and the axis of the underlying rotation are symbolized $\bar{n}$, in order to recall the composition $\mathcal{I} \circ \mathcal{R}(\hat{u}, 2 \pi / n)$. Additional rules, below discussed, allow inferring the orientation $\hat{u}$ of the rotation axes.

Any real orthogonal matrix $\alpha$ is unitary, to be explicit if $\alpha=\alpha^{\star}$ and $\alpha^{T} \alpha=l_{v}$ then $\alpha^{\dagger} \alpha=\left(\alpha^{\star}\right)^{T} \alpha=$ $\alpha^{T} \alpha=l_{\nu}$, where $\alpha^{\star}$ stands for the conjugate of $\alpha$ and $\alpha^{\dagger}$ for the adjoint of $\alpha$. This means that $\alpha$ is diagonalizable in the field $\mathbb{C}$ of complex numbers and that its eigenvalues $\left(\lambda_{1}, \ldots, \lambda_{v}\right)$ must satisfy the constraints $\left(\lambda_{k}\right)^{\star} \lambda_{k}=1$ and $\operatorname{Det}(\alpha)=\lambda_{1} \ldots \lambda_{v}= \pm 1$. These imply that $\lambda_{k}=e^{i \varphi_{k}}$ and that if $\varphi_{k} \neq$ $n \pi\left(\lambda_{k} \neq \pm 1\right)$ then there exists $\lambda_{q}=e^{i \varphi_{q}}$ in the series of the eigenvalues such that $\varphi_{q}=-\varphi_{k}$, namely the non real eigenvalues necessarily are paired by complex conjugates. Each pair can be interpreted as the eigenvalues of a 2-by-2 real orthogonal matrix of the type

$$
\rho\left(\mathcal{R}_{k}\right)=\left(\begin{array}{rr}
\cos \varphi_{k} & -\sin \varphi_{k} \\
\sin \varphi_{k} & \cos \varphi_{k}
\end{array}\right)
$$

associated with a rotation $\mathcal{R}_{k}$ in a 2-dimensional subspace $\mathcal{E}_{k}$ of $\mathcal{E}_{v}$. Indeed, the characteristic polynomial of $\rho\left(\mathcal{R}_{k}\right)$ is $\operatorname{Det}\left[\rho\left(\mathcal{R}_{k}\right)-x l_{2}\right]=x^{2}-2 x \cos \varphi_{k}+1=0$ the roots of which are $x_{ \pm}=$ $\cos \varphi_{k} \pm \sqrt{\cos ^{2} \varphi_{k}-1}=e^{ \pm i \varphi_{k}}$. A similarity transformation then exists block-diagonalizing the matrix $\alpha$ into the direct sum $\Pi \alpha \Pi^{-1}=\rho\left(\mathcal{R}_{1}\right) \oplus \cdots \oplus \rho\left(\mathcal{R}_{r=(\nu-p-q) / 2}\right) \oplus-l_{q} \oplus l_{p}$, where $\Pi$ is a non singular matrix of basis change and $\rho\left(\mathcal{R}_{1}\right), \cdots, \rho\left(\mathcal{R}_{r}\right)$ are 2-by-2 real orthogonal matrices associated with rotations in $(v-p-q) / 2$ mutually orthogonal 2-dimensional subspaces of $\mathcal{E}_{v}$. As an example, the real orthogonal matrices associated with the four symmetry operations depicted in figure 1 are given, 
with respect to the already well adapted $\mathrm{O} x y z$ frame $\left(\Pi=\iota_{3}\right)$, by

$$
\begin{aligned}
& \alpha(\overline{1})=\left(\begin{array}{rrr}
-1 & 0 & 0 \\
0 & -1 & 0 \\
0 & 0 & -1
\end{array}\right) \\
& \alpha(n)=\left(\begin{array}{rrr}
\cos \varphi & -\sin \varphi & 0 \\
\sin \varphi & \cos \varphi & 0 \\
0 & 0 & 1
\end{array}\right) \quad \text { with } \quad \varphi=\frac{2 \pi}{n} \\
& \alpha(\bar{n})=\left(\begin{array}{rrr}
\cos \varphi & -\sin \varphi & 0 \\
\sin \varphi & \cos \varphi & 0 \\
0 & 0 & -1
\end{array}\right) \quad \text { with } \quad \varphi=\frac{2 \pi}{n}+\pi \\
& \alpha(m)=\left(\begin{array}{rrr}
1 & 0 & 0 \\
0 & 1 & 0 \\
0 & 0 & -1
\end{array}\right)
\end{aligned}
$$

The set of the $v$-by- $v$ real orthogonal matrices equipped with the standard matrix multiplication law, to which the composition of the orientation symmetries in $\mathcal{E}_{v}$ transposes, makes up the orthogonal matrix group $\mathrm{O}(\nu, \mathbb{R})$ : i- the set is closed under matrix multiplication, to be precise $\forall(\alpha, \beta) \in$ $\mathrm{O}(\nu, \mathbb{R})^{2}, \forall(i, j),(\alpha \beta)_{i j}^{T}=(\alpha \beta)_{j i}=\sum_{k} \alpha_{j k} \beta_{k i}=\sum_{k} \beta_{k i} \alpha_{j k}=\sum_{k} \beta_{i k}^{T} \alpha_{k j}^{T}=\left(\beta^{T} \alpha^{T}\right)_{i j}$ thus $(\alpha \beta)^{T} \alpha \beta=$ $\beta^{T} \alpha^{T} \alpha \beta=\beta^{T} \imath_{v} \beta=\imath_{v}$ so $\alpha \beta \in \mathrm{O}(v, \mathbb{R})$; ii- matrix multiplication in general is associative, so it is within $\mathrm{O}(v, \mathbb{R})$; iii- the unit element $\imath_{v}$ of matrix multiplication is inside $\mathrm{O}(v, \mathbb{R})$; iv- any $\alpha$ in $\mathrm{O}(v, \mathbb{R})$ possesses an inverse $\alpha^{-1}$, since $\operatorname{Det}(\alpha)= \pm 1 \neq 0$, which belongs to $\mathrm{O}(\nu, \mathbb{R})$, since $\alpha^{T}=\alpha^{-1}$ and $\left(\alpha^{T}\right)^{T}=\alpha$ so that $\left(\alpha^{-1}\right)^{T} \alpha^{-1}=l_{\nu} . \mathrm{O}(\nu, \mathbb{R})$ is a subgroup of the group of $\nu$-by- $v$ real invertible matrices, the general linear group $\mathrm{GL}(\nu, \mathbb{R}){ }^{1}$

\subsubsection{Crystallographic restriction}

A crystal basically is an atomic stacking with finite periodicity so that if it is invariant under the rotation $\mathcal{R}(\hat{u}, \varphi)_{A}$ by an angle $\varphi$ about an $u$-axis crossing a point $A$ then necessarily an infinite number of points $B$ exists in the plane perpendicular to the $u$-axis at each of which the crystal is invariant under the rotation $\mathcal{R}(\hat{u}, \varphi)_{B}$ by the same angle about the same $u$-axis, now crossing the chosen point $B$. It goes without saying that the crystal then is invariant also under the rotations $\mathcal{R}(\hat{u},-\varphi)_{X}(X=A$ or $B)$, since $\mathcal{R}(\hat{u},-\varphi)_{X} \circ \mathcal{R}(\hat{u}, \varphi)_{X}=\imath_{3}$. Under $\mathcal{R}(\hat{u}, \varphi)_{A}$ the point $B$ is transformed into the point $B^{\prime}$ whereas under $\mathcal{R}(\hat{u},-\varphi)_{B}$ the point $A$ is transformed into the point $A^{\prime}$, as displayed in figure 2 -a. The quadruplet of points $\left(A, B, A^{\prime}, B^{\prime}\right)$ forms a parallelogram the three sides $(A B),\left(A B^{\prime}\right)$ and $\left(A^{\prime} B\right)$ of which have the same length $T$, since $B^{\prime}$ is the transform of $B$ by an isometry with fixed point $A$ and $A^{\prime}$ is

\footnotetext{
${ }^{1} \mathrm{O}(v, \mathbb{R})$ is a topological group of dimension $v(v-1) / 2$. It inherits its topology from the single canonical separated topology consistent with the structure of normed vector space of the set of $v$-by- $v$ real matrices $\mathrm{M}(v, \mathbb{R})$. This is of dimension $v^{2}$, since a $v$-by- $v$ matrix has $v^{2}$ independent entries, whereas the condition of orthogonality $\alpha^{T} \alpha=l_{v}$ gives rise to $v+v(v-1) / 2$ constraints. $\mathrm{O}(v, \mathbb{R})$ is compact because it is closed, being the inverse image of $l_{n}$ by the continuous application $\mathrm{M}(\nu, \mathbb{R}) \rightarrow$ $\mathrm{M}(\nu, \mathbb{R}): \alpha \mapsto \alpha^{T} \alpha$, and bounded, as inferred by considering the norm $\|\alpha\|=\left\{\operatorname{Tr}\left[\alpha^{T} \alpha\right]\right\}^{1 / 2} . \mathrm{O}(\nu, \mathbb{R})$ is path disconnected, which means that there exists matrices in the group manifold that cannot be connected by a smooth path. It more precisely is partitioned into two connected components: $\mathrm{O}(v, \mathbb{R})=\mathrm{SO}(v, \mathbb{R}) \times\left\{l_{v},-l_{v}\right\}$, where the normal subgroup $\mathrm{SO}(v, \mathbb{R})$ is the special orthogonal matrix group made up of the $v$-by- $v$ real orthogonal matrices with determinant +1 . SO $(v, \mathbb{R})$ is 2 -connected, which means that the closed paths in the group manifold cannot all be deformed into each other and form 2 homotopic classes. Its universal covering is the compact simply connected spinor group $\operatorname{Spin}(v)$. A familiar example is the spinor group Spin(3), which is isomorphic to the group $\mathrm{S}_{3}$ of unit quaternions and to the special unitary group $\mathrm{SU}(2, \mathbb{C})(\mathbb{C}$ is the field of complex numbers). A more detailed description of these topological properties would lead us too far from the scope of these notes. A wide literature exists on the topic. The interested reader might start for instance with J. Stillwell, Naive Lie Theory (Springer Science, 2008).
} 

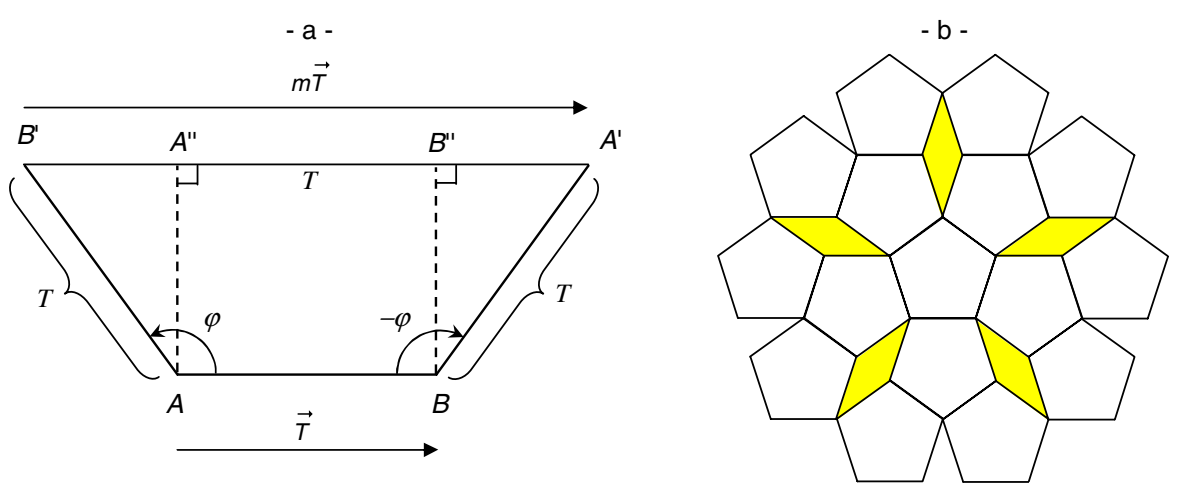

Figure 2. (Color online) a- Geometrical constraint on crystallographic rotations (see text). b- Visualization of the incompatibility of the rotation of order 5 with discrete translation - No way exists to pave the plane by regular pentagons without overlap nor gap (shown in yellow (gray) in the figure).

the transform of $A$ by an isometry with fixed point $B$. The fourth side $\left(A^{\prime} B^{\prime}\right)$ is parallel to the side $(A B)$ and has the length $T^{\prime}=T+2 T \sin (\varphi-\pi / 2)=T(1-2 \cos \varphi)$. The primed points are crystallographically equivalent to the unprimed ones, since the considered rotations are invariance operations, which implies that the vector $\vec{T}^{\prime}$ joining the former must be an integral multiple of the vector $\vec{T}$ joining the latter, namely an integer m must exist such that $\vec{T}^{\prime}=\mathrm{m} \vec{T}$. It follows that the angle of rotation $\varphi$ must satisfy the constraint: $1-2 \cos \varphi=\mathrm{m}$ or else $\cos \varphi=(1-\mathrm{m}) / 2$. This is solved solely for $(\varphi, \mathrm{m})=( \pm 2 \pi / 1,-1),( \pm 2 \pi / 6,0),( \pm 2 \pi / 4,1),( \pm 2 \pi / 3,2),( \pm 2 \pi / 2,3)$ (modulo $2 \pi$ for $\varphi)$. Accordingly, identifying the identity with $\mathcal{R}(\hat{u}, \varphi=2 \pi / 1)_{X} \forall(\hat{u}, X)$, the only proper rotations consistent with the finite periodicity of the 3-dimensional crystals are those of orders 1, 2, 3, 4 and 6.

Another way to find the same result is by inventorying the regular tessellations of the plane, namely by searching which convex regular $n$-gons can be used as a single unit to pave the plane without overlaps nor gaps. A regular $n$-gon is a polygon with all sides of equal length (equilateral) and all corners of equal angles (equiangular). It geometrically typifies an invariance with respect to a proper rotation of order $n$ whereas a tessellation intrinsically is periodic. Conversely, on any planar periodic pattern the same Wigner-Seitz convex cell can be defined on each element of a set of periodic congruent points by drawing lines normal to the lines joining a selected point to its nearest neighbors, thus materializing a contiguous cover of the plane. If in addition the periodic pattern is invariant under a rotation of order $n$ then the cell necessarily is a regular $n$-gon. Among evident examples are the regular tessellation based on equilateral triangles $(n=3)$, on squares $(n=4)$ or on regular hexagons $(n=6)$. An $n$-gon is defined solely for $n \geq 3$, but the tessellation with rectangles although not regular implies that the rotation symmetry of order 2 also is not forbidden. No tessellation with regular pentagons $(n=5)$ in contrast is possible, as tentatively pictured in figure 2-b, nor with any other regular $n$-gons. Indeed, each vertex of a regular tessellation is shared by an integral number $\mathrm{m}$ of $n$-gons so, to avoid any overlap or gap, the interior angle of the regular $n$-gon at a vertex necessarily must be $\theta_{n \text {-gon }}=2 \pi / \mathrm{m}$, but the sum of the interior angles of an $n$-gon is $(n-2) \pi$, as straightforwardly inferred on dividing the $n$-gon into $(n-2)$ contiguous triangles by drawing its diagonals from a selected vertex, so that $\theta_{n \text {-gon }}=(n-2) \pi / n$. It follows that $n$ must satisfy the constraint: $2 n /(n-2)=\mathrm{m}$, which is solved solely for $(n=3, \mathrm{~m}=6)$, $(n=4, \mathrm{~m}=4)$ and $(n=6, \mathrm{~m}=3)$. $\mathrm{m}$ always decreases for increasing $n$ so if $n>6$ then $\mathrm{m} \leq 2$, but $\mathrm{m}=2$ leads to $n=n-2$, which is absurd, and $\mathrm{m}=1$ to $n=-2$, which is meaningless.

A proof of algebraic nature often is provided too, making use of the similarity invariance of the matrix trace: $\forall \alpha \& \forall \Pi$ with $\operatorname{Det}(\Pi) \neq 0, \operatorname{Tr}\left(\Pi \alpha \Pi^{-1}\right)=\operatorname{Tr}(\alpha)$, where $\Pi$ would account for any change of basis vectors. If $\alpha$ is a real orthogonal matrix with determinant +1 associated with a rotation 
$\mathcal{R}(\hat{u}, \varphi)$ then an orthogonal frame $\mathrm{O} x_{1} x_{2} x_{3}$ exists where it takes the form displayed in eq. (2.2), which immediately allows establishing that $\operatorname{Tr}(\alpha)=\sum_{i} \alpha_{i i}=2 \cos \varphi+1$. If the frame is changed to another $\mathrm{O} \xi_{1} \xi_{2} \xi_{3}$ among those generating the lattice $\mathcal{L}_{\mathrm{O}}$ of points congruent to $\mathrm{O}$ by the finite translations inherent to a crystal then the rotation $\mathcal{R}(\hat{u}, \varphi)$ will be associated with a new matrix $\Pi \alpha \Pi^{-1}$ the entries of which must all be integers, otherwise $\mathcal{L}_{\mathrm{O}}$ will not be transformed into itself and the crystal will not be invariant under the rotation $\mathcal{R}(\hat{u}, \varphi)$. It follows that $\operatorname{Tr}\left(\Pi \alpha \Pi^{-1}\right)=\operatorname{Tr}(\alpha)=2 \cos \varphi+1$ must be an integer, which again limits the allowed values of the rotation angles $\varphi$ to $2 \pi / n(n=1,2,3,4,6)$ (modulo $2 \pi$ ).

On general grounds, an orientation symmetry is consistent with translation invariance in a discretized space of dimension $v$ if and only if there exists an invertible $v$-by- $v$ integer matrix of finite order to which it can be associated by similarity transformation: ${ }^{2}$ any orientation symmetry $\mathfrak{S}$ within a discretized space of dimension $v$ must be of finite order $\mathfrak{O} \mathfrak{r d}(\mathfrak{S})$, otherwise from a given position not belonging to the subspace of the fixed points of $\mathfrak{S}$ and at a finite distance from the fixed point $\mathrm{O}$ an infinite number of equivalent positions would be generated at the same distance by indefinite repeated applications of $\mathfrak{S}$, making up a dense set on a $(v-1)$-sphere in contradiction with a discrete geometry. A translation invariance in a discretized space is meant merely for the existence of a finite space periodicity. If this prevails along $v$ independent directions then a $v$-dimensional vector lattice implicitly is defined. An orientation symmetry $\mathfrak{S}$ will be consistent with the translation invariance if and only if it transforms the lattice of the nodes congruent to $\mathrm{O}$ into itself, in which case, selecting a frame $\mathrm{O} \xi_{1} \cdots \xi_{v}$ with basis vectors generating the vector lattice, it gets associated with an invertible $\nu$-by- $\nu$ integer matrix $\alpha_{\mathbb{Z}}$ of order $\mathfrak{O r d}\left(\alpha_{\mathbb{Z}}\right)=\min \left\{\eta \in \mathbb{N}^{\star} \mid \alpha_{\mathbb{Z}}^{\eta}=\imath_{\nu}\right\}=\mathfrak{O r d}(\mathfrak{S})$. It is recalled that an integer matrix is invertible if and only if its inverse exists and is an integer matrix. Its determinant then can be only \pm 1 . The set of the invertible $v$-by- $v$ integer matrices equipped with the standard matrix multiplication law makes up the matrix group $\operatorname{GL}(v, \mathbb{Z})$.

\subsubsection{How to obtain and name the 3-dimensional crystallographic point groups?}

A set of orientation symmetries consistent with translation invariance in a $v$-dimensional discretized space form a finite so-called point group, isomorphic to a subgroup of the matrix group GL( $v, \mathbb{Z})$, if and only if it is closed under symmetry composition. Any orientation symmetry indeed admits an inverse of the same order, since $\alpha_{\mathbb{Z}} \alpha_{\mathbb{Z}}^{-1}=l_{v}$ and $\alpha_{\mathbb{Z}}^{\eta}=l_{v}$ implies that $\left(\alpha_{\mathbb{Z}}^{-1}\right)^{\eta}=l_{v}$, thus eligible as a necessary element of the set to be closed. If all the elements of the set share the same subspace of fixed points then the point group is abelian and cyclic of order equal to the least common multiple of the orders of the involved orientation symmetries. Compositions of orientation symmetries not belonging to a single cyclic group also might lead to point groups, generally non abelian, depending on their mutual compatibility. Cataloging them is an old problem, which especially for $v=3$ might be dated back to the ancient Greeks.

It is convenient to inventory at first the point groups that can be generated solely from the proper rotations and to start by calling back some essential properties of these: if $\mathcal{R}(\hat{u}, \varphi)$ generically designates

\footnotetext{
2 The set $\operatorname{Ord}(v)=\left\{\mu \in \mathbb{N}^{\star} \mid \exists \alpha \in \mathrm{GL}(\nu, \mathbb{Z})\right.$ with $\left.\mathfrak{O} \mathfrak{r d}(\alpha)=\mu\right\}$ thus specifies the crystallographic restriction in arbitrary dimension $v$ in a nutshell ( $\mathbb{N}^{\star}$ is the set of strictly positive integers and $\mathbb{Z}$ is the ring of integers). Ord $(\alpha)=\min \{\eta \in$ $\left.\mathbb{N}^{\star} \mid \alpha^{\eta}=l_{v}\right\}$. It has been shown that $\operatorname{Ord}(v)=\{\mu \mid \Phi(\mu) \leq v\}$, where $\Phi$ is an additive version of Euler's totient function $\phi$ [3]: i- if $p$ is a prime then $\Phi\left(p^{k}\right)=\phi\left(p^{k}\right)=p^{k}-p^{k-1} \forall k \in \mathbb{N}^{\star}$; ii- if $r$ and $s$ are co-prime then $\Phi(r s)=\Phi(r)+\Phi(s)$ unless $r=2$ and $s$ is odd in which case $\Phi(2 s)=\Phi(s)-$ to compare with $\phi(r s)=\phi(r) \phi(s)$. $\forall v, \operatorname{Ord}(v) \subseteq \operatorname{Ord}(v+1)$ and, $\Phi(\mu)$ being even, $\forall \mu>2, \operatorname{Ord}(2 \nu)=\operatorname{Ord}(2 \nu+1)$. It follows that the elements of Ord(v) can be enumerated from those of $\operatorname{ord}(\nu)=\operatorname{Ord}(v)-\operatorname{Ord}(\nu-1)=\{\mu \mid \Phi(\mu)=\nu\}$ for $v$ even. ord $(\nu)$ moreover can be computed from the set $\operatorname{ord}^{\mathrm{o}}(v)$ of its odd elements, the set $\operatorname{ord}^{\mathrm{e}}(\nu)$ of its even elements being obtained from the theorem: $\operatorname{ord}^{\mathrm{e}}(v)=2 \operatorname{ord}^{\mathrm{o}}(\nu) \cup$ $\left[\cup_{k} 2^{k+1} \operatorname{ord}^{\mathrm{o}}\left(v-2^{k}\right)\right]\left(k \geq 1 \& 2^{k} \leq v\right)$. ord $(0)$ is set to $\{1\} . \operatorname{ord}(1)=\{2\} \cdot \operatorname{ord}(2 \eta+1)=\{\} \forall \eta \geq 1 . \operatorname{ord}(2)=\{3,4,6\}, \operatorname{ord}(4)=$ $\{5,8,10,12\}, \operatorname{ord}(6)=\{7,9,14,15,18,20,24,30\}, \operatorname{ord}(8)=\{16,21,28,36,40,42,60\}, \ldots$ so $\operatorname{Ord}(2)=\operatorname{Ord}(3)=\{1,2,3,4,6\}$, $\operatorname{Ord}(4)=\operatorname{Ord}(5)=\{1,2,3,4,5,6,8,10,12\}, \operatorname{Ord}(6)=\operatorname{Ord}(7)=\{1,2,3,4,5,6,7,8,9,10,12,14,15,18,20,24,30\}, \ldots$
} 


\section{Contribution of Symmetries in Condensed Matter}

a proper rotation of angle $\varphi$ about an $u$-axis crossing the point $\mathrm{O}$ then

$$
\mathcal{R}(\hat{u}, \varphi) \overrightarrow{\mathrm{OM}}=\overrightarrow{\mathrm{OM}} \cos \varphi+\hat{u}(\hat{u} \cdot \overrightarrow{\mathrm{OM}})(1-\cos \varphi)+(\hat{u} \wedge \overrightarrow{\mathrm{OM}}) \sin \varphi
$$

The transformation exhibits invariance under the $(-\hat{u},-\varphi) \rightarrow(\hat{u}, \varphi)$ substitution so that $\mathcal{R}(\hat{u}, \varphi)=$ $\mathcal{R}(-\hat{u},-\varphi)$. It also may be established, after elementary algebraic manipulations, that

$$
\mathcal{R}(\hat{u},-\varphi)[\mathcal{R}(\hat{u}, \varphi) \overrightarrow{\mathrm{OM}}]=\overrightarrow{\mathrm{OM}} \quad \forall \mathrm{M}
$$

which means that $\mathcal{R}(\hat{u},-\varphi)$ is the inverse with respect to symmetry composition of $\mathcal{R}(\hat{u}, \varphi)$, in agreement with the common intuition. Any composition $\mathcal{R}(\hat{u}, \varphi)=\mathcal{R}\left(\hat{u}_{2}, \varphi_{2}\right) \circ \mathcal{R}\left(\hat{u}_{1}, \varphi_{1}\right)$ of proper rotations is fully determined from the formulas:

$$
\begin{gathered}
\cos \frac{\varphi}{2}=\cos \frac{\varphi_{1}}{2} \cos \frac{\varphi_{2}}{2}-\left(\hat{u}_{1} \cdot \hat{u}_{2}\right) \sin \frac{\varphi_{1}}{2} \sin \frac{\varphi_{2}}{2} \\
\sin \frac{\varphi}{2} \hat{u}=\sin \frac{\varphi_{1}}{2} \cos \frac{\varphi_{2}}{2} \hat{u}_{1}+\sin \frac{\varphi_{2}}{2} \cos \frac{\varphi_{1}}{2} \hat{u}_{2}-\sin \frac{\varphi_{1}}{2} \sin \frac{\varphi_{2}}{2}\left(\hat{u}_{1} \wedge \hat{u}_{2}\right)
\end{gathered}
$$

which immediately show that the composition is commutative if and only if $\hat{u}_{1} \wedge \hat{u}_{2}=0$, namely the axes of the composed rotations are co-aligned $\left(\hat{u}_{1} \| \hat{u}_{2}=0\right)$ or anti-aligned $\left(\hat{u}_{1} \|-\hat{u}_{2}\right)$, in which case $\varphi=\varphi_{1}+\varphi_{2}$ or $\varphi_{1}-\varphi_{2}$, respectively. What however these equations above all indicate is that if the rotation angles are subject to crystallographic restrictions then there will be severe compatibility constraints on the respective orientations of the rotation axes. Composing for instance a flip $\left(\varphi_{1}=\pi\right)$ about a $u_{1}$-axis with a flip $\left(\varphi_{2}=\pi\right)$ about a $u_{2}$-axis at the angle $\theta_{1 \rightarrow 2}$ from the $u_{1}$-axis, we get a rotation of angle $2 \theta_{1 \rightarrow 2}$ about the $u$-axis of unit vector $\hat{u}_{1} \wedge \hat{u}_{2} / \sin \theta_{1 \rightarrow 2}$ :

$$
\mathcal{R}\left(\hat{u}_{2}, \varphi_{2}=\pi\right) \circ \mathcal{R}\left(\hat{u}_{1}, \varphi_{1}=\pi\right)=\mathcal{R}\left(\hat{u}=\hat{u}_{1} \wedge \hat{u}_{2} / \sin \theta_{1 \rightarrow 2}, \varphi=2 \theta_{1 \rightarrow 2}\right)
$$

It follows that the composition of the two flips would be inconsistent with any translation invariance if the angle $\theta_{1 \rightarrow 2}$ between the flip axes differs from $\pi / n(n=2,3,4,6)$ (modulo $\pi$ ). Now, if $\theta_{1 \rightarrow 2}$ takes one of these values then

$$
\mathcal{R}\left(\hat{u}, 2 \theta_{1 \rightarrow 2}\right) \circ \stackrel{(n-2)}{\cdots} \circ \mathcal{R}\left(\hat{u}, 2 \theta_{1 \rightarrow 2}\right)=\mathcal{R}(\hat{u}, 2 \pi / 1)
$$

which together with $\mathcal{R}\left(\hat{u}_{1}, \pi\right) \circ \mathcal{R}\left(\hat{u}_{1}, \pi\right)=\mathcal{R}(\hat{u}, 2 \pi / 1)=\mathcal{R}\left(\hat{u}_{2}, \pi\right) \circ \mathcal{R}\left(\hat{u}_{2}, \pi\right)$ defines a dihedral group $\mathcal{D}_{n}$ of order $2 n$, from its two generators $a_{1}=\mathcal{R}\left(\hat{u}_{1}, \pi\right)$ and $a_{2}=\mathcal{R}\left(\hat{u}_{2}, \pi\right)$ of order 2 : $\mathcal{D}_{n}=\left\langle a_{1}, a_{2}\right| a_{1}^{2}=a_{2}^{2}=e,\left(a_{1} a_{2}\right)^{n}=e$ (unit element) $\rangle_{(n>1)}$. Another abstract equivalent definition, making use of a generator $a$ of order 2 and a generator $b$ of order $n$, is $\mathcal{D}_{n}=$ $\left\langle a, b \mid a^{2}=e, b^{n}=e, a b=b^{n-1} a\right\rangle$. It reveals that the cyclic group $\mathcal{C}_{n}=\left\langle b \mid b^{n}=e\right\rangle$ is a normal subgroup of $\mathcal{D}_{n}$, symbolically $\mathcal{C}_{n} \triangleleft \mathcal{D}_{n}$, since $a b=b^{n-1} a \leftrightarrow a b a^{-1}=b^{-1}$ so that $\forall x \in \mathcal{C}_{n}, \forall y \in$ $\mathcal{D}_{n}, y x y^{-1} \in \mathcal{C}_{n}$. It follows that $\mathcal{D}_{n}$ is isomorphic to the semi-direct product of cyclic groups $\mathcal{C}_{n} \rtimes_{\psi} \mathcal{C}_{2}$, with respect to the action $\psi$ of $\mathcal{C}_{2}$ on $\mathcal{C}_{n}$ that associates the non trivial involution $a$ of $\mathcal{C}_{2}$ to the automorphism $\mathcal{C}_{n} \rightarrow \mathcal{C}_{n}: \zeta \mapsto a \zeta a^{-1}=\zeta^{-1}$. If $n$ is even and $n>2$ then the elements of order 2 in $\mathcal{D}_{n}$ get gathered into two distinct conjugacy classes and $\mathcal{D}_{n}$ can be split into two subgroups isomorphic to $\mathcal{D}_{n / 2}$. If $n$ is odd then all the elements of order 2 in $\mathcal{D}_{n}$ get gathered into a single conjugacy class. The conjugate of a proper rotation $\mathcal{R}\left(\hat{u}_{1}, \varphi_{1}\right)$ by any other proper rotation $\mathcal{R}\left(\hat{u}_{2}, \varphi_{2}\right)$ by definition is the proper rotation $\mathcal{R}\left(\hat{u}_{2}, \varphi_{2}\right) \circ \mathcal{R}\left(\hat{u}_{1}, \varphi_{1}\right) \circ \mathcal{R}\left(\hat{u}_{2},-\varphi_{2}\right)$. Using brute algebra to get its axis and its angle would be too cumbersome. It is more clever to observe that $\mathcal{R}\left(\hat{u}_{2}, \varphi_{2}\right) \hat{u}_{1}$ is certainly a unit vector of the searched axis. This indeed is transformed by $\mathcal{R}\left(\hat{u}_{2},-\varphi_{2}\right)$ into $\hat{u}_{1}$ which is conserved by $\mathcal{R}\left(\hat{u}_{1}, \varphi_{1}\right)$ then transformed back to $\mathcal{R}\left(\hat{u}_{2}, \varphi_{2}\right) \hat{u}_{1}$ by $\mathcal{R}\left(\hat{u}_{2}, \varphi_{2}\right)$. It thus is invariant under the considered conjugation. A little of geometric intuition also suggests that the transform $\mathrm{N}$ by $\mathcal{R}\left(\hat{u}_{1}, \varphi_{1}\right)$ of any point $\mathrm{M}$ is transformed by $\mathcal{R}\left(\hat{u}_{2}, \varphi_{2}\right)$ into the transform $\mathrm{N}^{\prime}$ by $\mathcal{R}\left(\hat{u}_{2}, \varphi_{2}\right) \circ \mathcal{R}\left(\hat{u}_{1}, \varphi_{1}\right) \circ \mathcal{R}\left(\hat{u}_{2},-\varphi_{2}\right)$ of the transform $\mathbf{M}^{\prime}$ by $\mathcal{R}\left(\hat{u}_{2}, \varphi_{2}\right)$ of $\mathbf{M}$. Since isometries are under concern, this implies that the searched angle merely 
is $\varphi_{1}$. Accordingly,

$$
\mathcal{R}\left(\hat{u}_{2}, \varphi_{2}\right) \circ \mathcal{R}\left(\hat{u}_{1}, \varphi_{1}\right) \circ \mathcal{R}\left(\hat{u}_{2},-\varphi_{2}\right)=\mathcal{R}\left(\mathcal{R}\left(\hat{u}_{2}, \varphi_{2}\right) \hat{u}_{1}, \varphi_{1}\right)
$$

One recovers the result that any pair of conjugate elements of a finite group must be of the same order, symbolically $z=x y x^{-1} \leftrightarrow z^{n}=x y x^{-1} x y x^{-1} \cdots x y x^{-1}=x y \cdots y x^{-1}=x y^{n} x^{-1}$. It nevertheless should be emphasized that eq. (2.11) leads to a much stronger statement.

Now, let $\mathrm{G}$ be a point group of order $n_{\mathrm{G}}$ containing only proper rotations. Let $\mathcal{C}_{n}(\hat{u})$ be a cyclic subgroup of order $n$ and index $\left[\mathcal{G}: \mathcal{C}_{n}(\hat{u})\right]=n_{\mathrm{G}} / n$ in $\mathrm{G}$, generated from a selected rotation $\mathcal{R}(\hat{u}, \varphi)$ of order $n$ in G. It is always possible to partition the group $\mathrm{G}$ in terms of left cosets of $\mathcal{C}_{n}(\hat{u})$ :

$$
\mathrm{G}=a_{1} \mathcal{C}_{n}(\hat{u})+a_{2} \mathcal{C}_{n}(\hat{u})+\cdots+a_{n_{\mathrm{G}} / n} \mathcal{C}_{n}(\hat{u})
$$

The set $\left\{a_{1} \hat{u}, a_{2} \hat{u}, \cdots, a_{n_{\mathrm{G}} / n} \hat{u}\right\}$ forms an orbit of $n_{\mathrm{G}} / n$ distinct unit vectors, each associated with a cyclic group of the same order $n$. Applying $a_{i}$ on $\hat{u}$ indeed is equivalent to take the conjugates by $a_{i}$ of the elements of $\mathcal{C}_{n}(\hat{u}): \forall \mathcal{R}(\hat{u}, \varphi) \in \mathcal{C}_{n}(\hat{u}), a_{i} \mathcal{R}(\hat{u}, \varphi) a_{i}^{-1}=\mathcal{R}\left(a_{i} \hat{u}, \varphi\right)$ (see eq. (2.11)). Geometrically, the unit vector $\hat{u}$ connects the fixed point $\mathrm{O}$, common to all the proper rotations in $\mathrm{G}$, to a point $\mathrm{P}$ on the 2-dimensional sphere $\mathcal{S}_{2}$ of unit radius around O. P is named $n$-pole to call to mind the order $n$ of the proper rotation it concerns. An orbit $\left\{a_{1} \hat{u}, a_{2} \hat{u}, \cdots, a_{n_{\mathrm{G}} / n} \hat{u}\right\}$ is identified with a class $\left\{P_{1}, P_{2}, \cdots, P_{n_{\mathrm{G}} / n}\right\}$ of equivalent $n$-poles [4]. If $\mathcal{R}(\hat{u}, \varphi)$ is conjugate to its inverse $\mathcal{R}(-\hat{u}, \varphi)$ then the orbit will be associated to $n_{\mathrm{G}} / 2 n$ distinct axes of order $n$. If not, the two rotations will produce two distinct orbits each of which will be associated to $n_{\mathrm{G}} / n$ distinct axes of order $n$. However, these are the same for the two orbits, with unit vectors that merely are opposite by pairs, and thus are counted twice. It follows that if $\mathfrak{N}_{n}$ distinct orbits of unit vectors of proper rotations of order $n$ can be built in $\mathrm{G}$ then the number of effective and distinct proper rotations of order $n$ that these $\mathfrak{N}_{n}$ orbits would account for is $\left(n_{\mathrm{G}} / 2 n\right) \mathfrak{N}_{n}(n-1)$. Collecting all the orders $n$ of the proper rotations realized in $\mathrm{G}$, all the elements of the group, except the identity, are generated once and only once. Accordingly,

$$
n_{\mathrm{G}}-1=\sum_{n \geq 2} \frac{n_{\mathrm{G}}}{2 n} \mathfrak{N}_{n}(n-1)
$$

or else, by dividing both members of the equation by $n_{\mathrm{G}} / 2$,

$$
2\left(1-\frac{1}{n_{\mathrm{G}}}\right)=\sum_{n \geq 2} \mathfrak{N}_{n}\left(1-\frac{1}{n}\right)
$$

It is observed that

$$
\begin{gathered}
n_{\mathrm{G}} \geq 2 \Rightarrow 1 \leq 2\left(1-1 / n_{\mathrm{G}}\right)<2 \\
n \geq 2 \Rightarrow(1-1 / n) \mathfrak{N}_{n} \geq \mathfrak{N}_{n} / 2 \geq 1 / 2
\end{gathered}
$$

So there can be at most 3 distinct orbits, otherwise the second member of eq. (2.14) would be greater than or equal to 2 when the first member should be strictly smaller than 2 . A single orbit would imply that $2\left(1-1 / n_{\mathrm{G}}\right)=(1-1 / n)$ or else $n_{\mathrm{G}}=\left(2-n_{\mathrm{G}} / n\right)$ the only solution of which is $n_{\mathrm{G}}=n=1$. With two orbits of cardinal $n_{\mathrm{G}} / n_{i}(i=1,2)$ eq. (2.14) is simplified into $2=n_{\mathrm{G}} / n_{1}+n_{\mathrm{G}} / n_{2}$, which possesses no solution other than $n_{1}=n_{2}=n_{\mathrm{G}}$ and merely means that $\mathrm{G}$ is the cyclic group $\mathcal{C}_{n_{\mathrm{G}}}$. In the case of three orbits of cardinal $n_{\mathrm{G}} / n_{i}(i=1,2,3)$ eq. (2.14) is written more conveniently in the form

$$
1+\frac{2}{n_{\mathrm{G}}}=\frac{1}{n_{1}}+\frac{1}{n_{2}}+\frac{1}{n_{3}}
$$

which immediately reveals that at least one of the $n_{i}$ must be equal to 2 , otherwise the second member $1 / n_{1}+1 / n_{2}+1 / n_{3}$ of the equation would be less or equal to 1 when the first member $1+2 / n_{\mathrm{G}}$ is strictly greater than 1 . There is no loss in generality to set $n_{1}=2$ and assume that $n_{2} \leq n_{3}$, the $n_{i}$ being 


\section{Contribution of Symmetries in Condensed Matter}

interchangeable. An inspection of the equation, by increasing values of $n_{2}$ and for each value of $n_{2}$ by increasing values of $n_{3}$, then leads to the following enumeration:

- $n_{1}=2, n_{2}=2, n_{3}=n_{\mathrm{G}} / 2$, which corresponds to the dihedral group $\mathcal{D}_{n_{3}}$ of order $2 n_{3}$.

- $n_{1}=2, n_{2}=3, n_{3} \geq 3$, which imposes that $n_{\mathrm{G}}=12 n_{3} /\left(6-n_{3}\right)$ so $n_{3}$ can take 3 values:

$* n_{3}=3$, in which case $n_{\mathrm{G}}=12-$ Geometrically this corresponds to 1 class of 6 two-poles and 2 classes of 4 three-poles. The three-poles of each class must be equidistant to each other on $\mathcal{S}_{2}$, so form the corners of a regular tetrahedron, and are the antipodes of those of the other class. The two-poles are the projections from $\mathrm{O}$ on $\mathcal{S}_{2}$ of the centers of the 6 edges. $\mathrm{G}$ then is dubbed tetrahedral group.

$* n_{3}=4$, in which case $n_{\mathrm{G}}=24-$ Geometrically this corresponds to a class of 12 two-poles, a class of 8 three-poles and a class of 6 four-poles. The four-poles must be equidistant to each other on $\mathcal{S}_{2}$, so form the corners of a regular octahedron. It may be shown that the centers of its faces coincide with the three-poles whereas the centers of its edges with the two-poles. G then is dubbed octahedral group.

$* n_{3}=5$, in which case $n_{\mathrm{G}}=60-$ Geometrically this corresponds to a class of 30 two-poles, a class of 20 three-poles and a class of 12 five-poles. The five-poles must be equidistant to each other on $\mathcal{S}_{2}$, so form the corners of a regular icosahedron. It may be shown that the centers of its faces coincide with the three-poles whereas the centers of its edges with the two-poles. $G$ then is dubbed icosahedral group.

- $n_{1}=2, n_{2} \geq 4, n_{3} \geq n_{2}$ gives $1 / n_{1}+1 / n_{2}+1 / n_{3} \leq 1$ which cannot equate to $1+2 / n_{\mathrm{G}}>1$.

Attention is drawn to the fact that stricto sensu no crystallographic restriction was invoked to get the above catalog. It was only assumed that the group must be finite and can be partitioned into cosets of cyclic groups. One then can understand why no restriction exists on the order of the cyclic groups nor on the order of the dihedral groups and why the icosahedral group which involves proper rotations of order 5 are obtained. If crystallographic restriction is taken into consideration then only the group $\mathcal{C}_{1}$, consisting solely in the identity, the cyclic groups $\mathcal{C}_{2}, \mathcal{C}_{3}, \mathcal{C}_{4}, \mathcal{C}_{6}$, the dihedral groups $\mathcal{D}_{2}, \mathcal{D}_{3}, \mathcal{D}_{4}, \mathcal{D}_{6}$, the tetrahedral group $\mathcal{T}$ and the octahedral group $\mathcal{O}$ are allowed, which leaves us with only $\mathbf{1 1}$ possible point groups of proper rotations.

It remains, for completing the catalog of the point groups, to take into consideration the improper rotations $\mathcal{I} \circ \mathcal{R}(\hat{u}, \varphi)$. It is clear that any point group $\mathrm{G}$ should contain a set of proper rotations, eventually reduced to the identity, which, equipped with the composition law of the rotations, necessarily form a subgroup $G_{p}$ of $G$, since the composition of two proper rotations is a proper rotation and the reciprocal of a proper rotation is a proper rotation too. If $p$ is the number of proper rotations and $i$ the number of improper rotations in $\mathrm{G}$, then $i \geq p$ because the composition of the proper rotations by a same improper rotation, among the $i$, gives $p$ distinct improper rotations, and $p \geq i$, because the composition of the improper rotations by one of these gives $i$ distinct proper rotations, so $p=i$ which means that if $\mathrm{G}_{\mathrm{p}}$ is a proper subgroup of $\mathrm{G}$ then it always is of index 2. One then is in front of two alternatives:

* G explicitly contains the inversion through the origin $\mathcal{I}$, in which case, denoting $\mathcal{N}$ its unit element (the identity), $\mathcal{C}_{i} \equiv(\{\mathcal{N}, \mathcal{I}\}, \circ)$ makes up a subgroup of $\mathrm{G}$. Now, i- any element of $\mathrm{G}$ evidently is the composition either of $\mathcal{N}$ or of $\mathcal{I}$ with an element of $\mathrm{G}_{\mathrm{p}}$, ii- only the unit element is shared by $\mathcal{C}_{i}$ and $\mathrm{G}_{\mathrm{p}}$, iii- $\mathcal{C}_{i}$ is normal, being the union of two conjugacy classes, and $\mathrm{G}_{\mathrm{p}}$ is normal, being of index 2 . It follows from these 3 properties that $\mathrm{G}$ is isomorphic to the direct product $\mathrm{G}=\mathcal{C}_{i} \times \mathrm{G}_{\mathrm{p}}$. As from the 11 point groups of proper rotations one then gets $\mathbf{1 1}$ additional point groups containing the inversion:

$$
\begin{gathered}
\left(\mathcal{C}_{i}, \mathcal{C}_{2 h}, \mathcal{C}_{3 i}, \mathcal{C}_{4 h}, \mathcal{C}_{6 h}\right) \equiv \mathcal{C}_{i} \times\left(\mathcal{C}_{1}, \mathcal{C}_{2}, \mathcal{C}_{3}, \mathcal{C}_{4}, \mathcal{C}_{6}\right), \\
\left(\mathcal{D}_{2 h}, \mathcal{D}_{3 d}, \mathcal{D}_{4 h}, \mathcal{D}_{6 h}\right) \equiv \mathcal{C}_{i} \times\left(\mathcal{D}_{2}, \mathcal{D}_{3}, \mathcal{D}_{4}, \mathcal{D}_{6}\right), \quad \mathcal{T}_{h} \equiv \mathcal{C}_{i} \times \mathcal{T} \quad \text { and } \quad \mathcal{O}_{h} \equiv \mathcal{C}_{i} \times \mathcal{O}
\end{gathered}
$$


* $\mathrm{G}$ contains rotoinversions but not the inversion $\mathcal{I}$ itself. It then is insightful to expand $\mathrm{G}$ in cosets of $\mathrm{G}_{\mathrm{p}}: \mathrm{G}=\mathrm{G}_{\mathrm{p}}+\mathcal{A} \mathrm{G}_{\mathrm{p}}$, where the coset representative $\mathcal{A}=\mathcal{I} \circ \mathcal{R}(\hat{u}, \varphi)$ is selected among the rotoinversions of $\mathrm{G}$. It is clear that the proper rotation $\mathcal{R}(\hat{u}, \varphi)$ cannot belong to $\mathrm{G}$ and therefore no more to $\mathrm{G}_{\mathrm{p}}$, otherwise its inverse $\mathcal{R}(\hat{u},-\varphi)$ would belong to $\mathrm{G}$ so $\mathcal{A} \circ \mathcal{R}(\hat{u},-\varphi)=$ $\mathcal{I} \circ \mathcal{R}(\hat{u}, \varphi) \circ \mathcal{R}(\hat{u},-\varphi)=\mathcal{I}$ as well. It follows that the coset expansion $\mathrm{G}^{*}=\mathrm{G}_{\mathrm{p}}+\mathcal{R}(\hat{u}, \varphi) \mathrm{G}_{\mathrm{p}}$ defines a group of proper rotations $G^{*}$ of which $G_{p}$ is a subgroup of index 2 and to which $G$ is isomorphic. Thus, to list the point groups containing rotoinversions but not the inversion it suffices to expand the point groups of proper rotations in cosets of a subgroup of index 2, perform the relevant substitution of the coset representative and check that the as-built group is not already in the collection:

$$
\begin{aligned}
\text { 1. } & \mathcal{C}_{2}=\mathcal{C}_{1}+\mathcal{R}(\hat{u}, 2 \pi / 2) \mathcal{C}_{1} \longrightarrow \mathcal{C}_{1}+\mathcal{M}_{\hat{u}} \mathcal{C}_{1}=\mathcal{C}_{s} \\
\text { 2. } & \mathcal{C}_{4}=\mathcal{C}_{2}+\mathcal{R}(\hat{u}, 2 \pi / 4) \mathcal{C}_{2} \longrightarrow \mathcal{C}_{2}+\mathcal{M}_{\hat{u}} \circ \mathcal{R}(\hat{u}, 2 \pi / 4) \mathcal{C}_{2}=\mathcal{S}_{4} \\
\text { 3. } & \mathcal{C}_{6}=\mathcal{C}_{3}+\mathcal{R}(\hat{u}, 2 \pi / 6) \mathcal{C}_{2} \longrightarrow \mathcal{C}_{3}+\mathcal{I} \circ \mathcal{R}(\hat{u}, 2 \pi / 6) \mathcal{C}_{3}=\mathcal{C}_{3 h} \\
\text { 4. } & \mathcal{D}_{2}=\mathcal{C}_{2}\left(\hat{u}_{1} \wedge \hat{u}_{2}\right)+\mathcal{R}\left(\hat{u}_{1}, 2 \pi / 2\right) \mathcal{C}_{2}\left(\hat{u}_{1} \wedge \hat{u}_{2}\right) \longrightarrow \mathcal{C}_{2}\left(\hat{u}_{1} \wedge \hat{u}_{2}\right)+\mathcal{M}_{\hat{u}_{1}} \mathcal{C}_{2}\left(\hat{u}_{1} \wedge \hat{u}_{2}\right)=\mathcal{C}_{2 v} \\
\text { 5. } & \mathcal{D}_{3}=\mathcal{C}_{3}\left(\hat{u}_{1} \wedge \hat{u}_{2}\right)+\mathcal{R}\left(\hat{u}_{1}, 2 \pi / 2\right) \mathcal{C}_{3}\left(\hat{u}_{1} \wedge \hat{u}_{2}\right) \longrightarrow \mathcal{C}_{3}\left(\hat{u}_{1} \wedge \hat{u}_{2}\right)+\mathcal{M}_{\hat{u}_{1}} \mathcal{C}_{3}\left(\hat{u}_{1} \wedge \hat{u}_{2}\right)=\mathcal{C}_{3 v} \\
\text { 6. } & \mathcal{D}_{4}=\mathcal{C}_{4}\left(\hat{u}_{1} \wedge \hat{u}_{2}\right)+\mathcal{R}\left(\hat{u}_{1}, 2 \pi / 2\right) \mathcal{C}_{4}\left(\hat{u}_{1} \wedge \hat{u}_{2}\right) \longrightarrow \mathcal{C}_{4}\left(\hat{u}_{1} \wedge \hat{u}_{2}\right)+\mathcal{M}_{\hat{u}_{1}} \mathcal{C}_{4}\left(\hat{u}_{1} \wedge \hat{u}_{2}\right)=\mathcal{C}_{4 v} \\
\text { 7. } & \mathcal{D}_{6}=\mathcal{C}_{6}\left(\hat{u}_{1} \wedge \hat{u}_{2}\right)+\mathcal{R}\left(\hat{u}_{1}, 2 \pi / 2\right) \mathcal{C}_{6}\left(\hat{u}_{1} \wedge \hat{u}_{2}\right) \longrightarrow \mathcal{C}_{6}\left(\hat{u}_{1} \wedge \hat{u}_{2}\right)+\mathcal{M}_{\hat{u}_{1}} \mathcal{C}_{6}\left(\hat{u}_{1} \wedge \hat{u}_{2}\right)=\mathcal{C}_{6 v} \\
\text { 8. } & \mathcal{D}_{4}=\mathcal{D}_{2}+\mathcal{R}\left(\hat{u}=\hat{u}_{1} \wedge \hat{u}_{2}, 2 \pi / 4\right) \mathcal{D}_{2} \longrightarrow \mathcal{D}_{2}+\mathcal{M}_{\hat{u}} \circ \mathcal{R}(\hat{u}, 2 \pi / 4) \mathcal{D}_{2}=\mathcal{D}_{2 d} \\
\text { 9. } & \mathcal{D}_{6}=\mathcal{D}_{3}+\mathcal{R}\left(\hat{u}=\hat{u}_{1} \wedge \hat{u}_{2}, 2 \pi / 6\right) \mathcal{D}_{3} \longrightarrow \mathcal{D}_{3}+\mathcal{M} \mathcal{M}_{\hat{u}} \circ \mathcal{R}(\hat{u}, 2 \pi / 6) \mathcal{D}_{3}=\mathcal{D}_{3 h} \\
\text { 10. } & \mathcal{O}=\mathcal{T}+\mathcal{R}(\hat{u}, 2 \pi / 4) \mathcal{T} \longrightarrow \mathcal{T}+\mathcal{I} \circ \mathcal{R}(\hat{u}, 2 \pi / 4) \mathcal{T}=\mathcal{T} d
\end{aligned}
$$

One thus gets $\mathbf{1 0}$ more point groups, containing rotoinversions but not the inversion. As to avoid being too lengthy the description of these is quite succinct and condensed into a notation that, it is hoped, should provide the necessary tools to guess the involved symmetries. Complements can be found in Ref. [5], while the detailed descriptions of the point groups, including their symbols (Hermann-Mauguin and Schoenflies) can be consulted in Ref. [1].

As a matter of fact, essentially the Schoenflies symbols were used above to designate the point groups, of which the rules merely are: $\mathrm{i}$ - find the most symmetric axis and take it vertical to define either a cyclic group $\mathcal{C}_{n}$ or a dihedral group $\mathcal{D}_{n}$; ii- use the specific symbol $\mathcal{C}_{i}$ for the group with only the identity and the inversion, $\mathcal{C}_{s}$ for the group with only the identity and the mirror, $\mathcal{T}$ for the tetrahedral groups and $\mathcal{O}$ for the octahedral groups; iii- add the index $i$ if the group involves the inversion but no mirror, the index $h$ if there is a horizontal mirror, the index $v$ if there is a vertical mirror, implicitly assuming that it crosses a horizontal flip axis if any, or the index $d$ if there is a vertical mirror bisecting two horizontal flip axes.

The international (Hermann-Mauguin) symbols by far are the most widely used, being preferred because the notation is implicitly associated with a lattice so is straightforwardly extended to the space groups. The full symbol of a point group is built by: i-identifying the symmetry axes if any that might work as the primary, secondary or tertiary directions of the least symmetric conventional cell (see subsection 2.2.1) invariant by the point group, ii-arranging side by side the symbols ( $n$ for a proper rotation, $\bar{n}$ for a rotoinversion or $m$ for a mirror) corresponding to the symmetry elements existing possibly along the primary, secondary and tertiary direction. If a mirror $m$ is perpendicular to a rotation axis $n$, thus bearing the same direction which for a mirror is its normal, then this is emphasized by a slash between the two symbols, to be precise through the notation $n / m$. The primary, secondary and tertiary directions must not be equivalent (by symmetry) and must be arranged by decreasing or equal symmetry order - except for the tetrahedral groups 23 and $2 / m \overline{3}$, to avoid confusion with the dihedral groups 32 and $\overline{3} 2 / m$ and to evoke a connection with the octahedral groups. A secondary direction exists as soon as some other symmetry element exists along another direction (in addition to that along the primary direction), while a tertiary direction exists as soon as some additional symmetry element 


\section{Contribution of Symmetries in Condensed Matter}

Table 1. The 32 crystallographic point groups ( $4^{\text {th }}$ column $)$ and the 11 Laue classes $\left(5^{\text {th }}\right.$ column $)$, as identified with the international (Hermann-Mauguin) symbol, full and short, respectively. Note that the full and short international symbols are exactly the same for all non symmetrical point groups and differ only for 6 Laue classes out of 11 . The $6^{\text {th }}$ column displays the order of each Laue class. The point groups are gathered according to the order of the symmetry element (order of the rotation, if larger or equal to 2, otherwise order of the reflection, 2) along the primary, secondary and tertiary directions (given in columns 1,2 and 3, where the symbol ' - ' indicates the absence of a direction).

\begin{tabular}{c|c|c|l|l|r}
\hline \hline \multicolumn{2}{c}{$\begin{array}{c}\text { Order of point symmetry along: } \\
\text { primary } \\
\text { direction }\end{array}$} & $\begin{array}{c}\text { secondary } \\
\text { direction }\end{array}$ & $\begin{array}{c}\text { tertiary } \\
\text { direction }\end{array}$ & \multicolumn{1}{c|}{$\begin{array}{c}\text { Crystallographic } \\
\text { point groups } \\
\text { (full symbol) }\end{array}$} & \multicolumn{2}{c}{$\begin{array}{c}\text { Laue class: } \\
\text { short } \\
\text { symbol }\end{array}$} & \begin{tabular}{c} 
order \\
\hline-
\end{tabular} & - & - & $1, \overline{1}$ & $\overline{1}$ & 2 \\
\hline 2 & - & - & $2, m, \frac{2}{m}$ & $2 / m$ & 4 \\
\hline 2 & 2 & 2 & $222,2 m m, \frac{2}{m} \frac{2}{m} \frac{2}{m}$ & $m m m$ & 8 \\
\hline 3 & - & - & $3, \overline{3}$ & $\overline{3}$ & 6 \\
3 & 2 & - & $32,3 m, \overline{3} \frac{2}{m}$ & $\overline{3} m$ & 12 \\
\hline 4 & - & - & $4, \overline{4}, \frac{4}{m}$ & $4 / m$ & 8 \\
4 & 2 & 2 & $422,4 m m, \overline{4} 2 m, \frac{4}{m} \frac{2}{m} \frac{2}{m}$ & $4 / m m m$ & 16 \\
\hline 6 & - & - & $6, \overline{6}, \frac{6}{m}$ & $6 / m$ & 12 \\
6 & 2 & 2 & $622,6 m m, \overline{6} 2 m, \frac{6}{m} \frac{2}{m} \frac{2}{m}$ & $6 / m m m$ & 24 \\
\hline 2 & 3 & - & $23, \frac{2}{m} \overline{3}$ & $m \overline{3}$ & 24 \\
4 & 3 & 2 & $432, \overline{4} 3 m, \frac{4}{m} \overline{3} \frac{2}{m}$ & $m \overline{3} m$ & 48 \\
\hline \hline
\end{tabular}

exists along another direction (in addition to that along the primary and secondary directions), assuming that this new direction is not equivalent by symmetry to the primary and secondary directions. ${ }^{3}$ The inversion never appears in the group symbol, except obviously in the case of the group $\overline{1}$, because it either is generated from the combination of a rotation of even order and a mirror, for instance $2 / \mathrm{m}$, or merely is implicitly contained in an elementary symmetry operation such as $\overline{3}$. It in fact is customary to rather make use of an abbreviate form of the symbol called the short symbol. This is obtained from the full symbol by omitting mirror or axis symbols, when these are perpendicular to each other and, of course, if the omitted symmetry element can be generated by those listed solely in the short symbol.

The catalog of the 32 crystallographic point groups, also named crystal classes, using the international full symbols, is displayed in Table 1. They are classified: i- in seven blocks from the top to the bottom, corresponding to the seven crystal systems (see subsection 2.2.1), that is to seven sets of primary, secondary and tertiary directions, going from the less to the most symmetrical, ii- from left to right in each line of the $4^{\text {th }}$ column, by increasing or equal point group order. The four last blocks are divided into two sets (on two different lines), the first set showing only a primary direction (or

\footnotetext{
3 As an example the point group symbolized $\mathcal{C}_{3 v}$ in Schoenflies notation contains a rotation of order 3 and three mirrors $m$ at $120^{\circ}$ from each other and containing the rotation axis 3 . Its international symbol is obtained as follows: the primary direction is that of the symmetry of the highest order, namely the rotation axis of order 3 . The three mirrors are perpendicular to axes in the plane perpendicular to the primary direction and at $120^{\circ}$ from each other. They are thus equivalent by symmetry (through the rotation of order 3 ), so that each of these three axes may correspond to the secondary direction. There is no tertiary direction. The international symbol then is $3 \mathrm{~m}$. The international symbol for the point group $\mathcal{C}_{4 v}$ by contrast is $4 \mathrm{~mm}$ : the $4 \mathrm{mirrors}$ are not all equivalent by the rotation of order 4 . They define two different directions, the secondary direction for a pair of perpendicular mirrors and the tertiary direction for the other pair, at $45^{\circ}$ from the secondary direction.
} 
primary plus secondary directions for the last block), the second set involving in addition secondary and (possibly) tertiary directions, thus being more symmetrical. The 11 groups containing the inversion (reported in the last position of each line in the fourth column) are particularized and called the 11 Laue classes. These are of crucial importance, since in a standard diffraction experiment, one can only probe the Laue class and not the point group: even if a crystal is not centrosymmetric, the diffraction pattern always displays an inversion symmetry so that one can access only to the product of the point group by the inversion. The Laue classes are explicitly displayed in the last column of Table 1 in their short symbol: they can be obtained by adding the inversion to any point group on the same line in the last but one column of the table, and consequently are those of higher order in the line. If one for instance performs a diffraction experiment on a crystal with point group $\overline{6} 2 m$ one would be able to extract only the information that it belongs to the Laue class $\frac{6}{m} \frac{2}{m} \frac{2}{m} \equiv 6 / m m m=\overline{6} 2 m \times \overline{1}$. The basic reason is that the phase of the scattering amplitude of photons or neutrons where the information on an eventual lack of inversion symmetry resides is lost when squaring the scattering amplitude with its conjugate to compare with the measured intensities. Anomalous scattering at an absorption edge should be considered to get an insight into the lack of inversion.

The transposition of the Hermann-Mauguin to the Schoenflies notation is performed as follows:

$$
\begin{aligned}
n \longleftrightarrow \mathcal{C}_{n}, \quad n 22 \stackrel{(\text { n even })}{\longleftrightarrow} \mathcal{D}_{n}, \quad 32 \longleftrightarrow \mathcal{D}_{3}, \quad 23 \longleftrightarrow \mathcal{T}, \quad 432 \longleftrightarrow \mathcal{O} \\
\overline{1} \longleftrightarrow \mathcal{C}_{i}, \quad \overline{3} \longleftrightarrow \mathcal{C}_{3 i} \equiv S_{6}, \quad \frac{n}{m} \stackrel{(n \text { even })}{\longleftrightarrow} \mathcal{C}_{n h}, \quad \frac{n}{m} \frac{2}{m} \frac{2}{m} \stackrel{(n \text { even })}{\longleftrightarrow} \mathcal{D}_{n h}, \\
\overline{3} \frac{2}{m} \longleftrightarrow \mathcal{D}_{3 d}, \quad \frac{2}{m} \overline{3} \longleftrightarrow \mathcal{T}_{h}, \quad \frac{4}{m} \frac{2}{m} \longleftrightarrow \mathcal{O}_{h} \\
m \longleftrightarrow \mathcal{C}_{s}, \quad \overline{4} \longleftrightarrow S_{4}, \quad \overline{6} \longleftrightarrow \mathcal{C}_{3 h}, \quad n m m \stackrel{(n \text { even })}{\longleftrightarrow} \mathcal{C}_{n v}, \quad 3 m \longleftrightarrow \mathcal{C}_{3 v}, \\
\overline{4} 2 m \longleftrightarrow \mathcal{D}_{2 d}, \quad \overline{6} 2 m \longleftrightarrow \mathcal{D}_{3 h}, \quad \overline{4} 3 m \longleftrightarrow \mathcal{T}_{d} .
\end{aligned}
$$

\subsubsection{Molecular symmetry}

A symmetry of a molecule designates any space transform that superposes the molecule into itself, more precisely that permutes its atoms of the same nature so that to leave it unchanged and without motion of its barycenter. It thus is an orientation symmetry by definition, the relevance of which might be essential: a number of the properties of an isolated molecule, chemical, vibrational, electric, spectroscopic, ..., might be predicted at a qualitative level from its symmetry group. In most instances it is not difficult to find which is this point group.

As an example, let us consider the molecule of ammonia $\mathrm{NH}_{3}$ depicted in figure 3-a. It is observed immediately that it displays a rotation symmetry axis of order 3 that goes through the nitrogen atom $\mathrm{N}$ and the barycenter of the three hydrogen atoms $\mathrm{H}$, to be precise it is invariant under the rotations of angle $2 s \pi / 3(s \in \mathbb{N})$ about this axis. Also evident are the three mirrors containing the axis of order 3 each crossing one of the three hydrogen atoms $\mathrm{H}$, namely the molecule is invariant under the reflection with respect to these planes. It is moreover clear that the normals to the three mirrors are equivalent by symmetry, interrelated by the rotation of order 3 which cyclically transforms each mirror into the two others. The rotation of order 3 being the symmetry of the highest order determines the primary direction whereas the normals to the mirrors (all equivalent by symmetry) determine the secondary direction (which thus consists of three axes at $120^{\circ}$ from each other). No other symmetry is displayed by the molecule. Accordingly, its point group in international notation is $3 \mathrm{~m}$.

As a next illustrative example, let us consider the molecule of sulfur hexafluoride $\mathrm{SF}_{6}$ depicted in figure 3-b. This is drawn in a cube, to evidence more clearly the various symmetry invariance of the 


\section{Contribution of Symmetries in Condensed Matter}

$-a-$

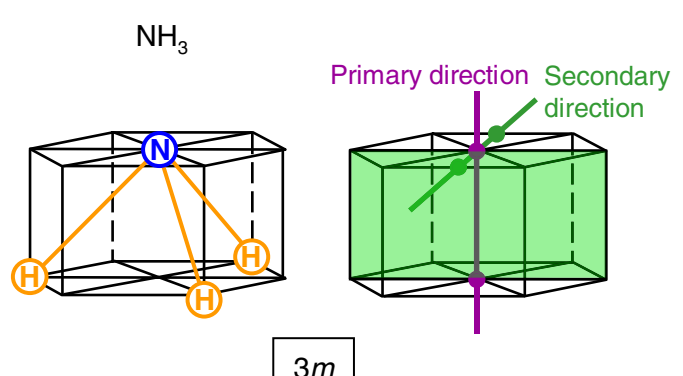

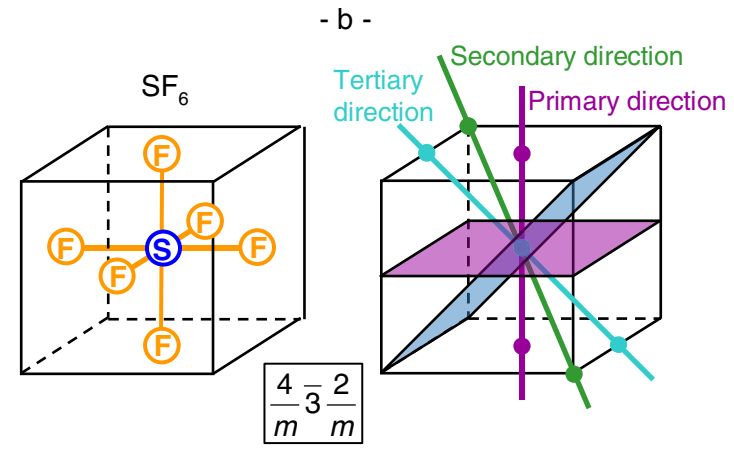

Figure 3. (Color online) Point group of two molecules: a- $\mathrm{NH}_{3}$, represented in a hexagonal basis prism, and b$\mathrm{SF}_{6}$, represented in a cube. Each molecule is drawn in the left part of the figure while the primary, secondary, and tertiary directions are shown in the right part. By sake of clarity, only one direction is plotted among the various equivalent directions for the secondary direction in the case of $\mathrm{NH}_{3}$ and for all directions in the case of $\mathrm{SF}_{6}$. Note that the tertiary direction does not exist for $\mathrm{NH}_{3}$. The full international symbol of the point group is given below each molecule.

molecule. One distinguishes 3 rotation axes of order 4 along the linear bonds $\mathrm{F}-\mathrm{S}-\mathrm{F}$ (parallel to the cube's edges) and the mirror planes perpendicular to them, 4 rotation axes of order 3 along the "long" diagonals of the cube (joining a corner of the cube to the opposite corner), 6 rotation axes of order 2 along the "short" diagonals of the cube (joining the middle of each edge to the middle of the opposite edge or merely diagonals of the cube faces) and the mirror planes perpendicular to them. It follows that the primary axis if of order 4 , the secondary axis of order 3 and the tertiary axis of order 2 . Only a little of geometric intuition is necessary to get convinced that the rotations of order 4 are equivalent by symmetry, interrelated by the rotations of order 3 about a same axis, and subsequently the mirrors perpendicular to them. Also equivalent are the rotations of order 3 , interrelated by the rotations of order 4 about a same axis, and similarly the rotations of order 2 and the mirrors perpendicular to them, interrelated by rotations of order 4 , rotations of order 3 or combinations of these. An evident symmetry finally is the inversion, which, combined with the rotations of order 3 , implies the invariance of the molecule under the rotoinversion $\overline{3}(\overline{3}=3 \times \overline{1})$. The full international symbol of the point group thus is $4 / m \overline{3} 2 / m$, which often is abbreviated into the short symbol $m \overline{3} m$.

It is emphasized that no crystallographic restriction applies to isolated molecules. It is thus not excluded to find molecules with symmetry invariance belonging to point groups other than those crystallographic. An example is the ferrocene molecule $\mathrm{Fe}\left(\mathrm{C}_{5} \mathrm{H}_{5}\right)_{2}$ the symmetry of which belongs to the dihedral group $\mathcal{D}_{5 d} \equiv \mathcal{C}_{i} \times \mathcal{D}_{5}$ (Schoenflies symbol). Another example is the buckyball fullerene molecule $\mathrm{C}_{60}$ the symmetry of which belongs to the full icosahedral group $\mathcal{I}_{h} \equiv \mathcal{C}_{i} \times \mathcal{I}$ (Schoenflies symbol) or $\frac{2}{m} \overline{3} \overline{5} \equiv \overline{1} \times 235$ (international symbol). This group is of order 120 and contains the icosahedral group $\mathcal{I}$ of order 60 as subgroup of index 2 .

\subsubsection{Stereographic projection}

A more convenient way than the one depicted in figure 1 to graphically represent the orientation symmetries of a point group is the stereographic projection, which also allows displaying the transformation by these symmetries of an initial arbitrary direction emerging from the fixed point $\mathrm{O}$ of the symmetries, so finding at a glance all the equivalent directions in a crystal.

A stereographic projection is a conformal mapping that projects the unit sphere $\mathcal{S}_{2}$ of center O from either the north pole $\mathrm{N}$ or the south pole $\mathrm{S}$ onto a plane perpendicular to the axis formed by the two poles, which implicitly will be assumed to be the equatorial plane for convenience. As a matter of fact, 

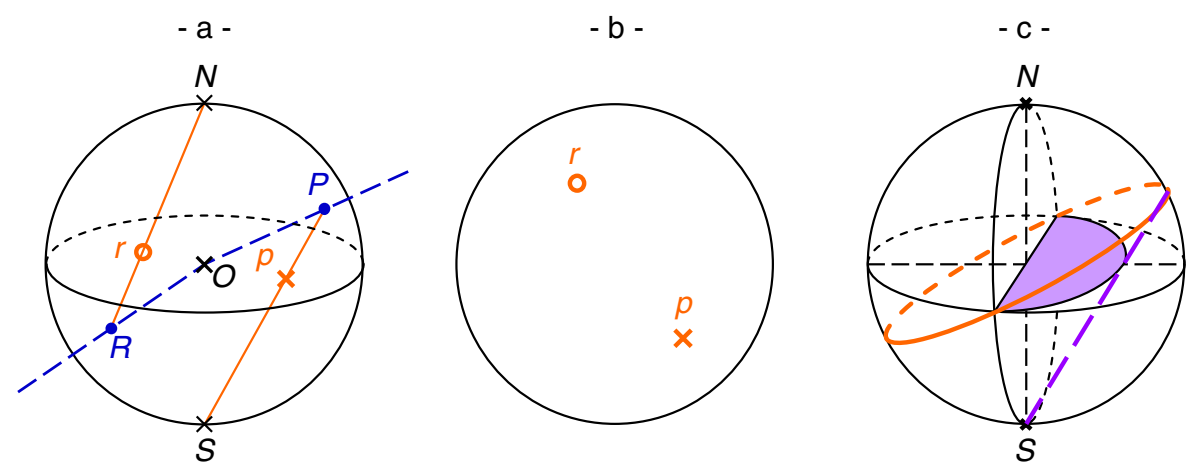

Figure 4. (Color online) a- Crossing points $\mathrm{P}$ and $\mathrm{R}$ of arbitrary directions (blue dashed half-lines) with the unit sphere $\mathcal{S}_{2}$ centered on the fixed point O, stereographic projection $p$ of the crossing point $\mathrm{P}$ on the north hemisphere from the south pole $\mathrm{S}$ and stereographic projection $r$ of the crossing point $\mathrm{R}$ on the south hemisphere from the north pole N. b- Equatorial section of the unit sphere $\mathcal{S}_{2}$ explicitly displaying the stereographic projection for directions OP and OR. c- Crossing circle (orange arc) of an inclined plane with the unit sphere $\mathcal{S}_{2}$ and stereographic projection (truncated elliptic contour of the equatorial surface in purple) of the portion of the circle on the north hemisphere from the south pole $\mathrm{S}$.

to gather all the information within the equatorial disk, the north hemisphere by convention is projected from the south pole and the south hemisphere is projected from the north pole, discerning the projected points from the north hemisphere by a cross marker $(x)$ and from the south hemisphere by a circle marker (o). This is illustrated in figure 4, for two arbitrary directions emerging from the point O, plotted in blue, intersecting the sphere $\mathcal{S}_{2}$ at the point $\mathrm{P}$ on the north hemisphere, projected onto the point $p$, and at the point $\mathrm{R}$ on the south hemisphere, projected onto the point $r$. The figure also describes the projection of the crossing of an inclined plane with the sphere. A mirror crosses the unit sphere $\mathcal{S}_{2}$ through a circle and thus projects in the equatorial plane through a line if it is vertical, through the equatorial circle if it is horizontal and through an ellipse if it is inclined.

The stereographic projections for the point groups $n$ and $\bar{n}$ are displayed figure 5: in these graphs both the symmetry operations and the equivalent directions are represented. The rotation axes by convention are identified with a filled cigar-shaped ellipse marker if it is of order $n=2$ and a filled $n$-gon marker if it is of order $n$ (black triangle for $n=3$, black square for $n=4$, black hexagon for $n=6)$. The inversion $(\overline{1})$ is discerned by an empty circle marker at the center of the equatorial circle. A mirror $(m=\overline{2})$ is represented by a thick straight line if it is perpendicular to the equatorial plane and a thick circle if it is parallel to the equatorial plane. The axis of the other rotoinversions finally are identified by a filled (for $n=3$ ) or an empty (for $n=4$ and 6) $n$-gon marker on which the marker(s) accounting for the other symmetry element(s) that the rotoinversion contains is (are) plotted: empty circle over filled triangle for $\overline{3}$, filled ellipse over empty square for $\overline{4}$ and filled triangle over empty hexagon together with thick circle for $\overline{6}$. The order of the point group is simply obtained by counting the total number of crosses and circles, except of course the empty circle at the origin, if any, signaling the inversion. One also immediately recognizes a Laue class, for then a cross is diametrically opposed to each circle and conversely in the stereographic projections. A quick look at figure 5 thus tells that $\overline{1}$ and $\overline{3}$ are Laue classes.

An efficient geometric intuition of the symmetries can be forged from the stereographic projections, but at the cost of a certain practice especially for the tetrahedral and octahedral point groups where inclined mirrors and rotation axes are involved. As to ease this, the manner in which the different symmetries that might exist in the cubic groups would project are suggested in figure 6 . The square marker in the middle of the right figure corresponds to the four-fold axis parallel to the $z$-axis, set parallel to the north-south axis of the unit sphere $\mathcal{S}_{2}$, while all square and ellipse markers plotted on 


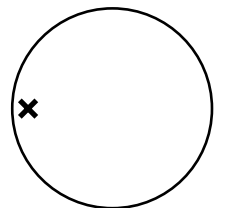

1

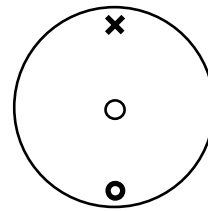

$\overline{1}$
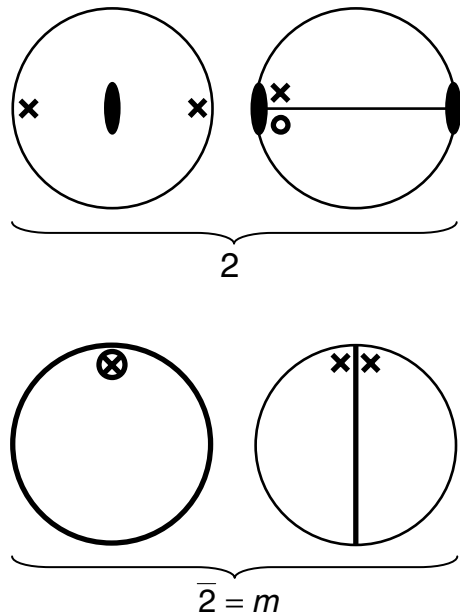

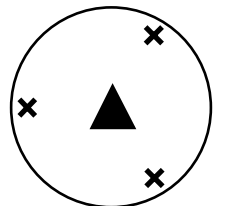

3

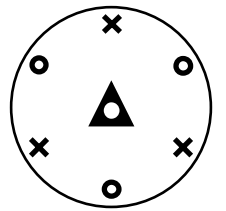

$\overline{3}$

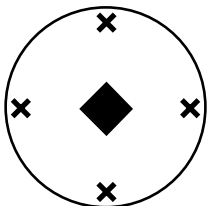

4

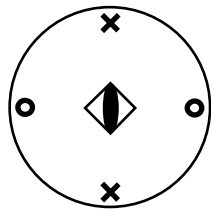

$\overline{4}$

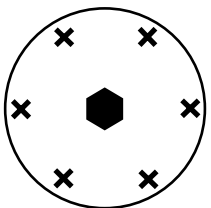

6

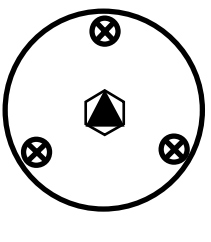

$\overline{6}=3 / m$

Figure 5. Graphs of stereographic projections for point groups $n$ and $\bar{n}$. There is no symmetry axis for groups 1 and $\overline{1}$. A symmetry axis exists for the other groups, which is single. It is set parallel to the north-south axis, except for the axis of order 2 . This is also set perpendicular to the north-south axis as a second convenient alternative, in particular for $\overline{2}$ with the mirror either in the equatorial plane or perpendicular to it. In all the cases one chooses an arbitrary point on the sphere $\mathcal{S}_{2}$ in the north hemisphere and considers its projection and that of its transforms by the symmetries.

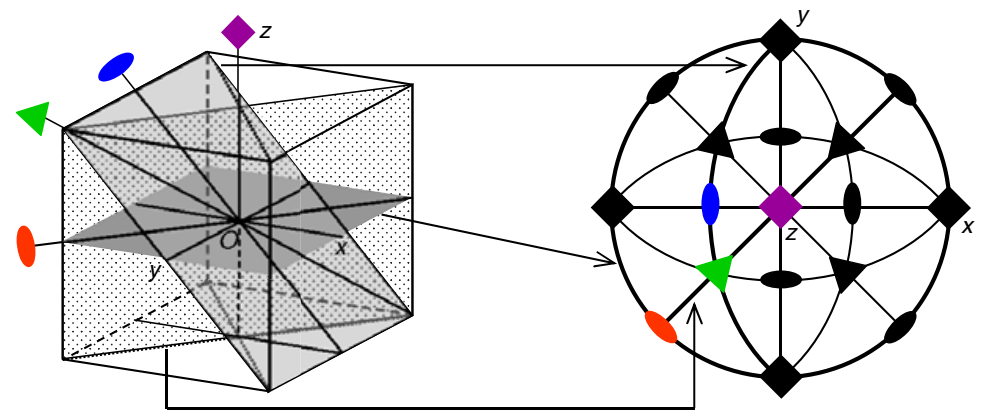

Figure 6. (Color online) Graphical support to perform and read stereographic projections for the cubic point groups. Left part: three-dimensional representation of a cube with particularization of a few planes and axes. Right part: stereographic projection of the cube will all possible mirror planes and rotation axes. A thick line is used for the three planes shown in gray in the left part of the figure and the same colored marker is used as for the symmetry axes particularized in the left part of the figure.

the equatorial circle are rotation axes lying in the $(x, y)$ plane of the cube perpendicular to the $z$-axis. The four straight lines correspond to the planes perpendicular to the $(x, y)$ plane. The four inclined symmetry planes of the cube give rise to the four truncated ellipses in the stereographic projection. It is easy to locate the inclined three-fold and two-fold axes from them. The three-fold axes are at the intersection of the three planes perpendicular to the cube's faces along their diagonals. Their markers in the stereographic projection are thus at the intersection of two truncated ellipses and one straight line along $x \pm y$. Similarly, the inclined two-fold axes are at the intersection of an inclined plane (truncated ellipse) and a plane parallel to the vertical faces of the cube (straight line along $x$ or $y$ ). Now, according to whether a symmetry operation exists or not in the specific cubic point group under consideration, the lines associated with the mirrors are to be drawn thick or thin and the filled or empty markers of the 

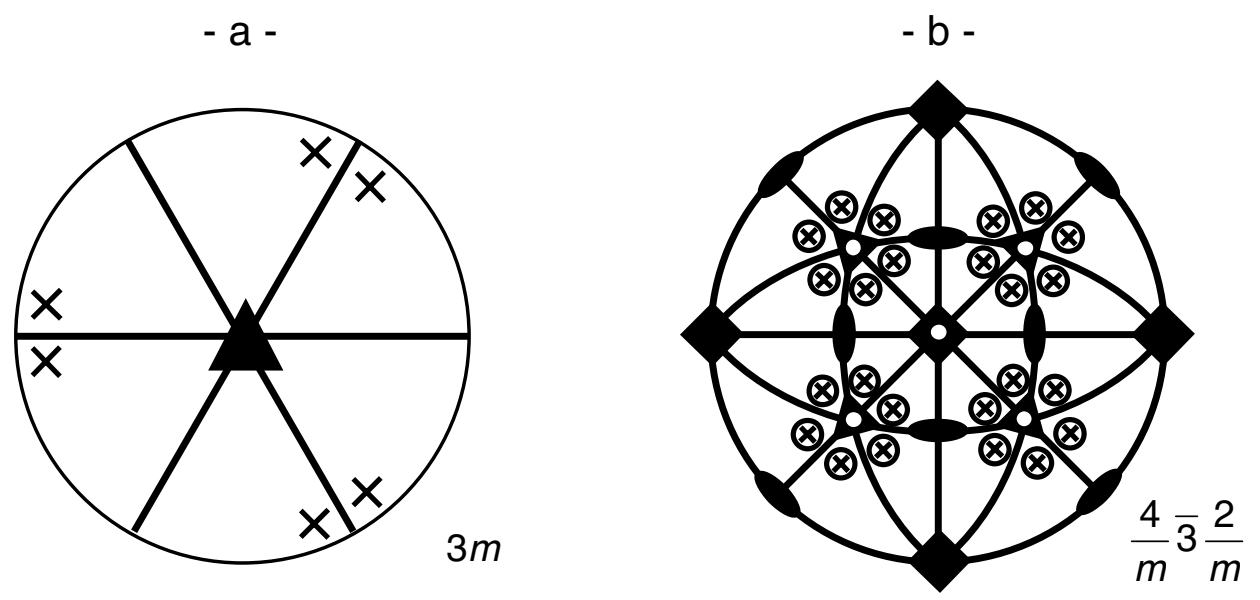

Figure 7. Stereographic projections for point groups $3 m$ (a) to which the $\mathrm{NH}_{3}$ molecule belongs and $4 / m \overline{3} 2 / m$ (short symbol: $m \overline{3} m$ ) (b) to which the $\mathrm{SF}_{6}$ molecule belongs (see also figure 3 ).

rotation or rotoinversion axes are to be appropriately substituted for those in the figure 6 or these merely should be erased.

Let us consider back the cases of the molecules $\mathrm{NH}_{3}$, belonging to point group $3 m$, and $\mathrm{SF}_{6}$, belonging to point group $m \overline{3} m . \mathrm{NH}_{3}$ contains a three-fold axis along the primary direction, set parallel to the north-south axis, and three mirrors at $120^{\circ}$ from each other and running through the primary axis. The secondary direction then might be along any of the $x, y$ and $-x-y$ direction at $120^{\circ}$ from each other in the equatorial plane, being equivalent by the three-fold symmetry axis. The stereographic projection is displayed in the figure $7-\mathrm{a} . \mathrm{SF}_{6}$ is described by the highest symmetrical point group, that is $4 / m \overline{3} 2 / m$ : all the symmetry elements of the cube shown in the figure 6 are thus present with, in addition, the inversion marker, an empty circle, at the center of the equatorial plane (see figure 7-b). The three-fold axes being that of rotoinversion $(\overline{3})$, an empty circle is also superposed to the triangular markers at their centers. A description of the stereographic projections for the 32 crystallographic point groups can be found in Part 10 of the International Tables for Crystallography, Volume A [1].

\subsubsection{What did the ancient Greeks know about orientation symmetry?}

A certain perception of the point groups in essence already existed among the ancient Greeks through the symmetry invariance of the platonic solids, so dubbed in reference to the philosopher Plato who, in the dialogue Timaeus, postulated that each of the assumed basic constitutive elements of the world displays a specific geometric shape, to be precise the tetrahedron for fire, the octahedron for air, the icosahedron for water, the cube for earth and the dodecahedron for aether (or universe as a whole). Euclid, in the Book XIII of the Elements, instructs us on the geometric construction of these polyhedra (represented in figure 8) and gets for each the edge length $e$ as a function of the diameter $d$ of the sphere in which it is inscribed: $e^{2}=(2 / 3) d^{2}$ for the tetrahedron, $e^{2}=(1 / 2) d^{2}$ for the octahedron, $e^{2}=(1 / 3) d^{2}$ for the cube, $e^{2}=(1 / 10)(5-\sqrt{5}) d^{2}$, the square of an irrational number called minor, for the icosahedron, and $e^{2}=(1 / 36)(\sqrt{15}-\sqrt{3})^{2} d^{2}$, the square of an irrational number called apotome, for the dodecahedron. Euclid ends the Book XIII by stating that no platonic solid other than the five inventoried might exist. Credited to the Greek mathematician Theaetetus contemporary to Plato, this finding is argued by establishing that with three equilateral triangles the tetrahedron vertex is constructed, with four the octahedron vertex and with five the icosahedron vertex, but six form plane angles and more than six are impossible to arrange contiguously. It next is observed that squares might meet only to form a cube 

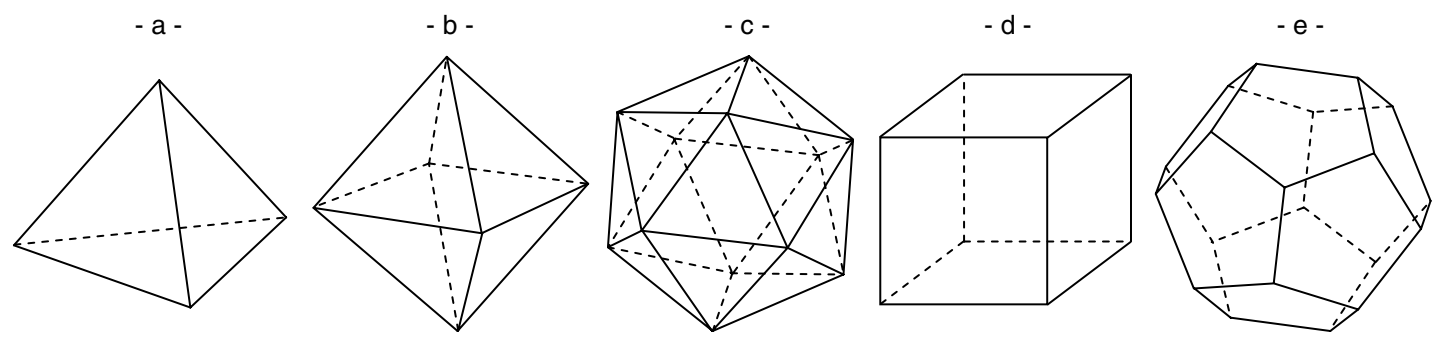

Figure 8. The five (regular) platonic solids: a- tetrahedron, b- octahedron, c- icosahedron, d- cube, and e- dodecahedron.

vertex and pentagons only to form a dodecahedron vertex. It finally is pointed out that no other regular polygons with more than five sides might meet to form a non planar solid angle.

A platonic solid, in the modern mathematical language, is a convex regular polyhedron in the 3-dimensional space, namely a polyhedron homeomorphic to a sphere the faces of which are regular $p$-gons all congruent by isometry to one another, with the faces intersecting solely at the edges and the same number $q$ of faces meeting at each vertex. It follows that its Euler characteristic is $\chi=$ $V-E+F=2$, where $V, E, F$ are the number of vertices, edges and faces. Each of its edges connects two vertices and shares two faces, so that $p F=2 E=q V$. Combining the two equations it is inferred that $1 / p+1 / q=1 / 2+1 / E>1 / 2$. This is solved solely for $\{p, q\}=\{3,3\},\{4,3\},\{3,4\},\{5,3\},\{3,5\}$, which proves the Theaetetus theorem ${ }^{4}$.

The vertices of the tetrahedron, the octahedron and the icosahedron were identified as the $n$-poles of highest order $n$ of the tetrahedral group $\mathcal{T}$, the octahedral group $\mathcal{O}$ and the icosahedral group $\mathcal{I}$ in this order (cf. eq. (2.15) and its solutions), which implicitly implies that these three platonic solids are invariant under these three respective groups of proper rotations. The centers of the faces of the platonic solids also were identified to form a class of $n$-poles of their invariance group of proper rotations. Now, by connecting these centers of faces one gets the dual polyhedron. Accordingly, this must belong to the same invariance group. The dual of the octahedron is the cube, which thus is invariant under the octahedral group $\mathcal{O}$, and the dual of the icosahedron is the dodecahedron, which thus is invariant under the icosahedral group $\mathcal{I}$. The tetrahedron is self-dual. If the improper symmetries are taken into consideration then the full symmetry group of the tetrahedron is $\mathcal{T}_{d}$, that of the octahedron and the cube is $\mathcal{O}_{h}=\mathcal{C}_{i} \times \mathcal{O}$, and that of the icosahedron and the dodecahedron is $\mathcal{I}_{h}=\mathcal{C}_{i} \times \mathcal{I}$. It finally may be called back that the cyclic group $\mathcal{C}_{n}$ and the dihedral group $\mathcal{D}_{n}$ are respectively the invariance group of proper symmetries and the symmetry group of the regular $n$-gon.

\subsection{Translation symmetry}

A 3-dimensional crystal by definition is a discrete medium where any point with its environment is spatially replicated an infinite discrete number of times along 3 independent directions. It would appear that this was formulated first by Bravais. As a matter of fact, this extends the familiar concept of space homogeneity to a discrete medium. The set of points congruent to a chosen point, that is the set of points

\footnotetext{
4 The generalization to arbitrary dimensions $v$ was examined. A platonic solid then is a convex regular $v$-polytope (2-polytope $\equiv$ polygon, 3-polytope $\equiv$ polyhedron). It has been shown that for $v \geq 5$ there can be only three different convex regular polytopes: the regular $\nu$-simplex, the measure polytope, also called $v$-cube or hypercube, and the cross polytope, also called $v$-cocube, $\nu$-orthoplex or hyperoctahedron, coinciding in dimension $v=3$ with the tetrahedron, the cube and the octahedron. It in contrast is found out that six convex regular polytopes exists in dimension $v=4$ : the 4-simplex, the 4-cube, the 4-cocube, the dodecaplex and the tetraplex, these two making up analogs of the icosahedron and the dodecahedron in 4 dimensions and the so-called 24-cell or octaplex with no analog in the other dimensions. The Euler characteristic of 4-polytopes is $\chi=\sum_{k}(-)^{k} \mathcal{F}_{k}=\chi\left(\mathcal{S}_{3}\right)=0$, where $\mathcal{F}_{k}$ is the number of $k$-faces $(0$-face $=$ vertex, 1 -face $=$ edge, $\ldots)$.
} 


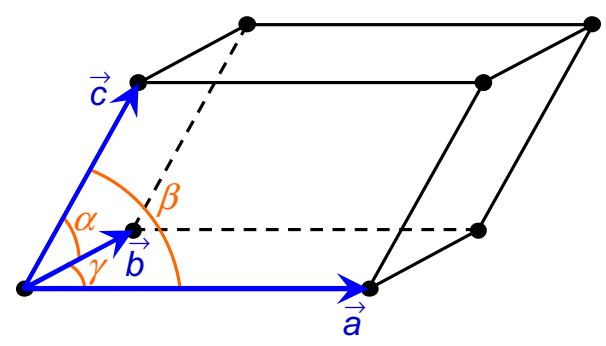

Figure 9. (Color online) The most general unit cell. The black points represent lattice points.

with a same given environment, determines a crystal lattice. A point congruent to the chosen point is also called a lattice point or a lattice node.

If $\vec{a}, \vec{b}$ and $\vec{c}$ are the shortest length vectors joining two lattice points along each of the 3 independent directions then, obviously, all the lattice points are reached from a chosen point by means of the translation vectors

$$
\vec{T}=u \vec{a}+v \vec{b}+w \vec{c}
$$

where $u, v, w$ are integers. The trihedron of non coplanar vectors $\vec{a}, \vec{b}, \vec{c}$ in eq. (2.16) forms a basis of the lattice. The parallelepiped these vectors define is called the unit cell. Figure 9 represents the most general unit cell that can be thought about. Its replication by the vectors $\vec{T}$ paves the whole space without empty space nor overlap. One by convention calls lattice parameters of the crystal associated with this unit cell the set formed by the lengths $a, b, c$ of the lattice basis vectors $\vec{a}, \vec{b}, \vec{c}$ and the angles $\alpha, \beta, \gamma$ between them, these being always defined in the following way [1]:

$$
\alpha=(\widehat{\vec{b}, \vec{c}}), \quad \beta=(\widehat{\vec{c}, \vec{a}}), \quad \gamma=(\widehat{\vec{a}, \vec{b}})
$$

A lattice point is found at each of the 8 corners of the unit cell, but each of them is shared by 8 different cells thus counting for $\frac{1}{8}$ in each cell. It follows that the number of lattice points per unit cell, called the multiplicity of the cell, is $m=8 \times \frac{1}{8}=1$. In the case of more symmetric crystal lattice, cells containing more than one lattice point are used (see next subsection), the so-called centered cells (doubly primitive, triply primitive, etc...). As opposed to them, a cell containing a single point is called a primitive cell, like the one represented in figure 9. Note that the use of a non primitive cell implies that additional vectors must be specified besides those defining the cell, in order to describe the complete lattice (see next subsection). All primitive cells have the same volume defined by the mixed product:

$$
V_{p}=(\vec{a}, \vec{b}, \vec{c})=(\vec{a} \wedge \vec{b}) \cdot \vec{c}=(\vec{b} \wedge \vec{c}) \cdot \vec{a}=(\vec{c} \wedge \vec{a}) \cdot \vec{b}
$$

whereas a non primitive cell has the volume $V_{m}=m V_{p}$, with $m$ the multiplicity of the cell.

At each lattice point is associated a group of atoms: the motif. The knowledge of the crystal lattice, more precisely of the trihedron of vectors $\vec{a}, \vec{b}, \vec{c}$ defining the unit cell, and that of the motif, more precisely the nature and fractional coordinates $x, y, z$ of the atoms in the unit cell, completely characterizes the crystalline structure.

The translation vectors $\vec{T}$ in the eq. (2.16) may also be interpreted as describing the translation symmetries of the crystal, that is the spatial translations that bring the crystal into coincidence with itself. The composition of the translation symmetries of a crystal is merely transposed to the ordinary vector addition of the corresponding translation vectors. The set of the translation vectors $\vec{T}$ of a crystal endowed with the vector addition form an infinite countable abelian group: the translation symmetry group of the crystal. 


\section{Contribution of Symmetries in Condensed Matter}

Table 2. The 6 conventional cells: symbol ( $1^{\text {st }}$ column), name ( $2^{\text {nd }}$ column $)$ and constraints on the lattice lengths ( $3^{\text {rd }}$ column $)$ and lattice angles ( $4^{\text {th }}$ column). The symbol '- ' in the last two columns means no constraint. The angles for which no constraint exists are different from $60^{\circ}, 90^{\circ}$ and $120^{\circ}$.

\begin{tabular}{c|c|c|c}
\hline \hline \multicolumn{2}{|c|}{ Conventional cell: } & \multicolumn{2}{c}{ Constraints on: } \\
symbol & name & lattice lengths & lattice angles \\
\hline$a$ & triclinic & - & - \\
\hline$m$ & monoclinic & - & $\alpha=\beta=90^{\circ}, \gamma>90^{\circ}$ \\
\hline$o$ & orthorhombic & - & $\alpha=\beta=\gamma=90^{\circ}$ \\
\hline$t$ & tetragonal or quadratic & $a=b$ & $\alpha=\beta=\gamma=90^{\circ}$ \\
\hline$h$ & hexagonal & $a=b$ & $\alpha=\beta=90^{\circ}, \gamma=120^{\circ}$ \\
\hline$c$ & cubic & $a=b=c$ & $\alpha=\beta=\gamma=90^{\circ}$ \\
\hline \hline
\end{tabular}

\subsubsection{The orientation symmetry of lattices: the 6 conventional cells, 7 crystal systems,} and 14 Bravais lattices

A crystal lattice must be consistent with any orientation symmetry existing in the crystal. If for instance the crystal shows an $n$-fold rotation symmetry then its axis is necessarily parallel to a lattice vector $\vec{u}$ and perpendicular to a lattice plane. Within this any lattice vector $\vec{v}$ is transformed into a lattice vector $\vec{w}$ with the same length and oriented at the angle $\frac{2 \pi}{n}$ from $\vec{v} .^{5}$ It is obvious that if $n \neq 2$ and if the shortest length lattice vectors along $\vec{v}, \vec{w}$ and $\vec{u}$ are chosen as the lattice basis vectors $\vec{a}, \vec{b}$ and $\vec{c}$ then the constraints $\alpha=90^{\circ}=\beta, a=b$ and $\gamma=360^{\circ} / n$ are imposed. The inversion symmetry is always present at the center of a unit cell, so that it suffices to consider the constraint inherent to each Laue class to get the catalog of all the possible unit cells consistent with orientation symmetry. As an example, the Laue class $2 / m$ imposes that one crystallographic axis called the unique axis, to which the axis 2 is parallel, must be perpendicular to the two others. Moreover, the angle between the two other axes has to be different from $90^{\circ}$, otherwise such a unit cell would correspond to the Laue class $\mathrm{mmm}$, and different from 60 or $120^{\circ}$, otherwise an axis of order 3 or 6 would exist and such a unit cell would thus correspond to another Laue class. Using similar arguments for each Laue class, one obtains a total of the $\mathbf{6}$ conventional unit cells, which are listed in Table 2.

The hexagonal unit cell splits in two different crystal systems, according to whether the rotation axis along the primary direction is of order 3 (trigonal system) or 6 (hexagonal system), while for all the other cells, conventional cells and crystal systems are the same, which leads to 7 crystal systems. Looking at Tables 1 and 2, the correspondence between the seven blocks of point groups (separated by horizontal lines in Table 1) and the seven crystal systems is straightforward and is explicitly detailed in Table 3 . This table also gives, for each crystal system and its associated point groups, the correspondence between the primary, secondary and tertiary directions and the crystallographic directions of the corresponding crystal system.

As explained in the previous subsection, a unit cell can be either primitive (symbol $P$, multiplicity $m=1$ ) or non primitive. Starting from the 6 conventional cells, one can consider, for each of them,

5 The axis of a rotation $\alpha$ by definition is the set of its fixed points. So any vector $\vec{u}$ along it belongs to $\operatorname{ker}\left(\alpha-\imath_{3}\right)$. On a basis $\{\vec{a}, \vec{b}, \vec{c}\}$ built over a crystal lattice, the matrix representative of $\left(\alpha-l_{3}\right)$ has integer entries. This implies that, as solutions of the homogeneous system of equations $\left(\alpha-\imath_{3}\right) \vec{u}=\overrightarrow{0}$, the components $u_{a}, u_{b}, u_{c}$ of the vectors $\vec{u} \in \operatorname{ker}\left(\alpha-\imath_{3}\right)$ are necessarily of the form $u_{a} / p_{a}=u_{b} / p_{b}=u_{c} / p_{c}$ where $p_{a}, p_{b}, p_{c}$ are integers. Thus, every rotation axis in a crystal is parallel to a lattice vector. Next, if $\vec{v}$ is a lattice vector not parallel to $\vec{u}$ then $\alpha(\vec{v})-\vec{v}$ is a lattice vector such that $\vec{u} \cdot(\alpha(\vec{v})-\vec{v})=0$. If $\vec{w}$ is a third lattice vector neither parallel to $\vec{u}$ nor to $\vec{v}$ then $\alpha(\vec{w})-\vec{w}$ is a lattice vector such that $\vec{u} \cdot(\alpha(\vec{w})-\vec{w})=0$ and $\alpha(\vec{w})-\vec{w}$ is not parallel to $\alpha(\vec{v})-\vec{v}$. Thus, every rotation axis in a crystal is perpendicular to a lattice plane. 


\section{EPJ Web of Conferences}

Table 3. The 7 crystal systems, their associated point groups, and the correspondence between the primary, secondary and tertiary directions and the crystallographic directions of the cell. Note that the hexagonal conventional cell (see Table 2) splits in two different crystal systems, trigonal and hexagonal, according to whether the primary direction corresponds to a rotation of order 3 or 6 , respectively.

\begin{tabular}{c|c|c|c|c}
\hline \hline Crystal system & Point groups & $\begin{array}{c}\text { primary } \\
\text { direction }\end{array}$ & $\begin{array}{c}\text { secondary } \\
\text { direction }\end{array}$ & $\begin{array}{c}\text { tertiary } \\
\text { direction }\end{array}$ \\
\hline triclinic & $1, \overline{1}$ & - & - & - \\
\hline monoclinic & $2, m, 2 / m$ & $\vec{b}$ & - & - \\
\hline orthorhombic & $222,2 m m, m m m$ & $\vec{a}$ & $\vec{b}$ & $\vec{c}$ \\
\hline trigonal & $3, \overline{3}$ & $\vec{c}$ & - & - \\
& $32,3 m, \overline{3} m$ & $\vec{c}$ & $\vec{a}, \vec{b},-\vec{a}-\vec{b}$ & - \\
\hline tetragonal & $4, \overline{4}, 4 / m$ & $\vec{c}$ & - & - \\
or quadratic & $422,4 m m, \overline{4} 2 m, 4 / m m m$ & $\vec{c}$ & $\vec{a}, \vec{b}$ & $\vec{a} \pm \vec{b}$ \\
\hline hexagonal & $6, \overline{6}, 6 / m$ & $\vec{c}$ & - & - \\
& $622,6 m m, \overline{6} 2 m, 6 / m m m$ & $\vec{c}$ & $\vec{a}, \vec{b}, \vec{a}+\vec{b}$ & $2 \vec{a}+\vec{b}, \vec{a}+2 \vec{b},-\vec{a}+\vec{b}$ \\
\hline cubic & $23, m \overline{3}$ & $\vec{a}, \vec{b}, \vec{c}$ & $\vec{a} \pm \vec{b} \pm \vec{c}$ & - \\
& $432, \overline{4} 3 m, m \overline{3} m$ & $\vec{a}, \vec{b}, \vec{c}$ & $\vec{a} \pm \vec{b} \pm \vec{c}$ & $\vec{a} \pm \vec{b}, \vec{b} \pm \vec{c}, \vec{c} \pm \vec{a}$ \\
\hline \hline
\end{tabular}

a primitive cell, and, for some of them, one can built some centered cells (by adding some lattice points), provided no symmetry element is lost and there exists no primitive cell describing the same lattice and having the same symmetry. This means that there exist only a few manners to center a cell, leading to the four additional lattice types listed below:

- body centered (additional lattice point at the center of the cell): symbol $I, m=2$;

- all-face centered (additional lattice points at the center of the 6 faces): symbol $F, m=4$;

- one-face centered (additional lattice points at the center of one pair of parallel faces): symbol $A, B$ or $C$, for the centered faces $(b, c),(a, c)$ or $(a, b)$, respectively, $m=2$;

- rhombohedral (additional lattice points at $1 / 3$ and $2 / 3$ of the long diagonal of the cell, that is at $(2 / 3,1 / 3,1 / 3)$ and $(1 / 3,2 / 3,2 / 3))$ : symbol $R, m=3$.

The rhombohedral lattice type exists only for the hexagonal conventional cell and is depicted in figure 10. In addition, the $R$-centering forces the primary axis to be of order 3 and thus applies only for the trigonal crystal system. For centered cells, additional lattice points exist at fractional coordinates, so that the set of translation vectors given by eq. (2.16) has to be expanded to a set of $m$ translation vectors (with $m$ the multiplicity of the cell) simply by adding the additional lattice points' coordinates to the translation vector $\vec{T}$. For example, a body-centered lattice will be fully described by translation vectors $\vec{T}_{1}=\vec{T}=u \vec{a}+v \vec{b}+w \vec{c}$ and $\vec{T}_{2}=\vec{T}+\left(\frac{1}{2}, \frac{1}{2}, \frac{1}{2}\right)=\left(u+\frac{1}{2}\right) \vec{a}+\left(v+\frac{1}{2}\right) \vec{b}+\left(w+\frac{1}{2}\right) \vec{c}$, while an all-face centered lattice will be fully described by $\vec{T}_{1}=\vec{T}, \vec{T}_{2}=\vec{T}+\left(\frac{1}{2}, \frac{1}{2}, 0\right), \vec{T}_{3}=\vec{T}+\left(0, \frac{1}{2}, \frac{1}{2}\right)$, and $\vec{T}_{4}=\vec{T}+\left(\frac{1}{2}, 0, \frac{1}{2}\right)$.

The centered lattice types can exist only for a restricted list of conventional cells, considering the constraints explained above. A triclinic lattice for instance can only be primitive, since, starting from any centered triclinic cell (associated with the Laue class: $\overline{1}$ ), one can always built a primitive triclinic cell of smaller volume, thus with the same symmetry. As another example, the tetragonal $C$ face centered cell does not exist, because one can then built a primitive tetragonal cell of smaller volume with $a^{\prime}=a / \sqrt{2}$ and $\vec{a}^{\prime}$ along the diagonal of the $(a, b)$ square face, nor do the tetragonal $A$ - and $B$-face 

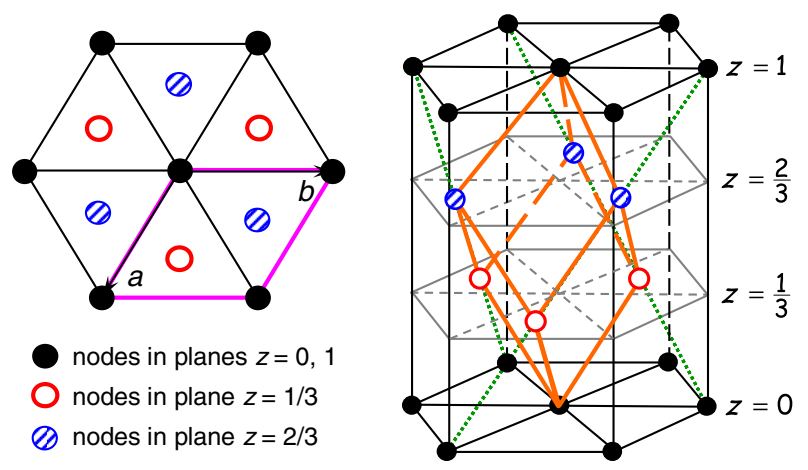

Figure 10. (Color online) The rhombohedral lattice type: projection in the $(a, b)$ plane (left figure) and perspective view (right figure). These figures show the position of the additional nodes of a rhombohedral hexagonal lattice (of multiplicity 3) evidencing the shape of its primitive cell: the rhombohedral cell (plotted in orange). Note that in the ITC [1], the description of space groups (see next section) is given in both cells.

Table 4. The 14 Bravais lattices, classified by conventional cell (vertically) and by lattice type (horizontally). Note that the $P$ hexagonal cell refers to the trigonal as well as the hexagonal crystal system, while the $R$ hexagonal cell refers only to the trigonal system.

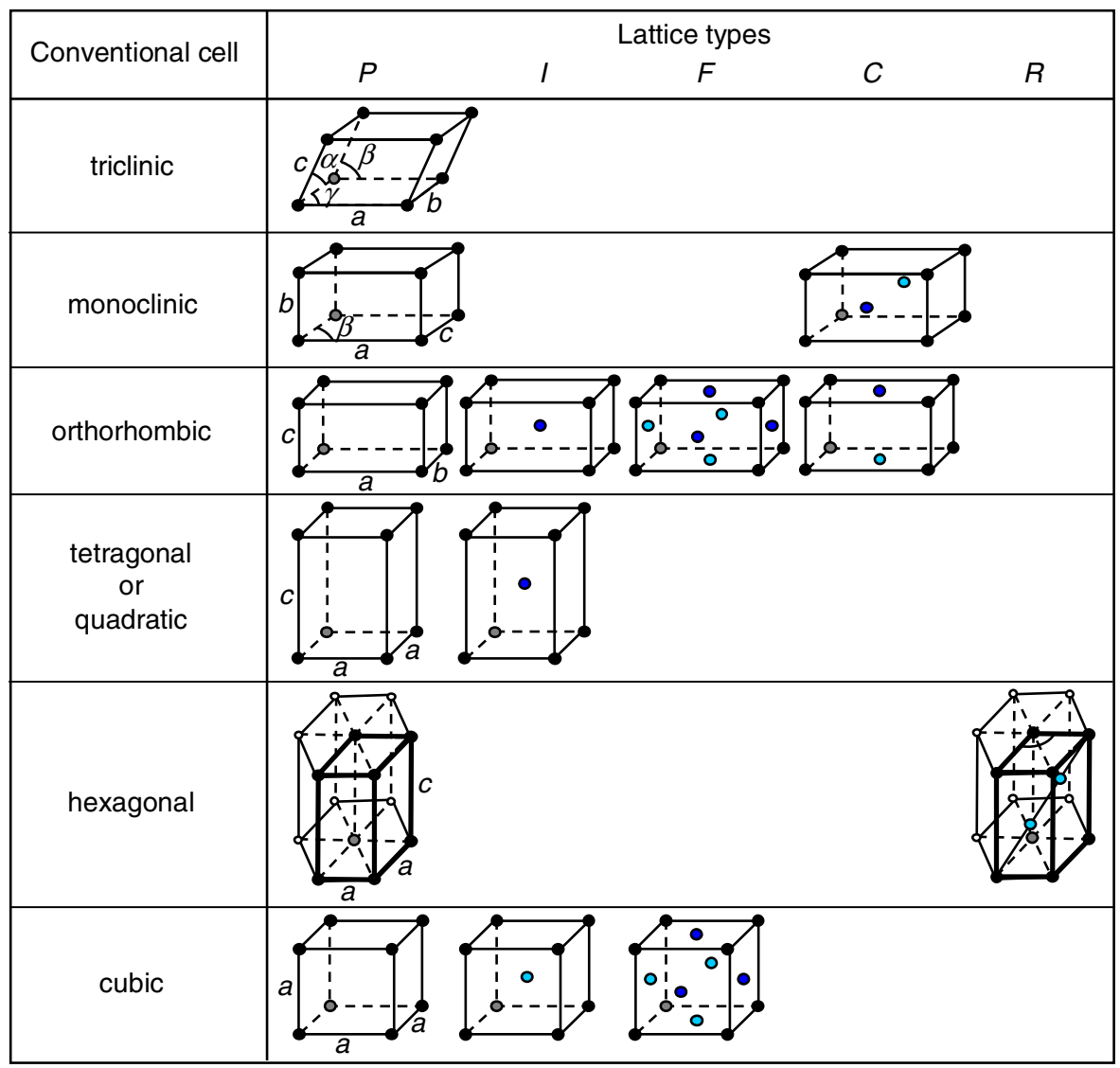



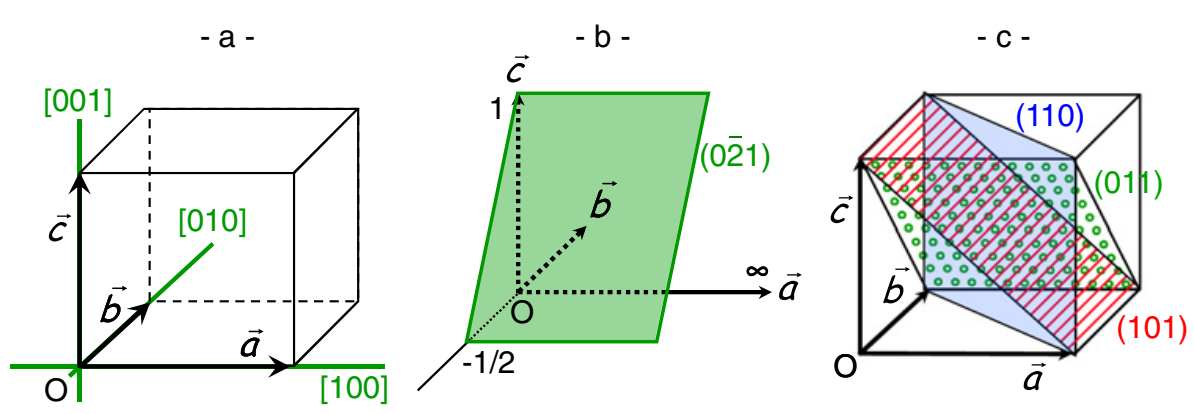

Figure 11. (Color online) a- Example of rows in a cubic unit cell. These three families of rows are equivalent, by symmetry, and thus belong to the same family $\langle 100\rangle$ (or, equivalently, $\langle 010\rangle$ or $\langle 001\rangle$ ), b- Example of net planes: the closest plane from the origin, plotted here, intercepts the $a, b$ and $c$ axes at $x=\infty, y=-1 / 2$ and $z=1$, respectively, so that the Miller indices are: $h=0, k=\overline{2}$ and $l=1$ (Note that in crystallography, minus signs are always placed above the number to which they apply: $\overline{2}$ means -2), c- Example of net planes in a cubic unit cell. These three families of net planes are equivalent, by symmetry, and thus belong to the same family $\{110\}$ (or, equivalently, $\{101\}$ or $\{011\})$.

centered tetragonal cells exist, because the 4-fold rotation axis would then be merely lost. As a matter of fact, from the 6 conventional cells one can build only the $\mathbf{1 4}$ Bravais lattices which are listed in Table 4 .

\subsubsection{Rows and net planes}

One can always group lattice points into parallel equidistant rows denoted [uvw] along $\vec{n}_{u v w}=$ $u \vec{a}+v \vec{b}+w \vec{c}$, with $u, v, w$ integers having no common divider. $n_{u v w}$ is called the parameter of the row, while $u, v$ and $w$ are called the indices of the row. A family of rows contains all lattice points. If families of rows are equivalent by symmetry then they are denoted $\langle u v w\rangle$. Figure 11-a shows an example of equivalent rows in a cubic unit cell.

In the same way, one can always group lattice points into parallel equidistant net planes, spaced by $d_{h k l}$ and denoted $(h k l)$, with $h, k, l$ integers having no common divider. The integers $h, k, l$ are called Miller indices and $d_{h k l}$ is called the d-spacing. These planes follow the equation: $h x+k y+l z=m$, so that the plane the closest from the origin ( $m=1$ ) intercepts the $\vec{a}, \vec{b}$ and $\vec{c}$ axes at $x=1 / h, y=1 / k$ and $z=1 / l$, respectively. The expression of $d_{h k l}$ as a function of the lattice parameters will be given in subsection 2.4.2, since it requires having introduced the reciprocal space. Family of net planes, equivalent by symmetry, are denoted $\{h k l\}$. Figures $11-\mathrm{b}$ and $11-\mathrm{c}$ show examples of net planes. The same property as for the rows holds: a family of net planes contains all lattice points.

We will come back to these notions of rows and planes in subsection 2.4.2, since they play a crucial role in diffraction, and particularly when relating the reciprocal space to the direct space.

\subsection{Space group symmetry}

Up to now, we have seen that a crystal is characterized by a lattice and a motif. More precisely, the complete description of a crystal requires: $i$ - the knowledge of the Bravais lattice (lattice parameters and lattice type, in order to define the set of the $m$ translation vectors, with $m$ the multiplicity), ii- the knowledge of the motif (nature and fractional coordinates of the $N$ atoms contained in a unit cell, for a $P$ lattice, or of the $N / m$ atoms contained in one half, one third, or one fourth of the unit cell, for a centered cell with $m=2,3$, or 4 , respectively). The lattice is completely defined by the translation symmetry, which always exists in crystals at a microscopic scale as discussed in Section 2.2, while there also exist symmetries acting inside the motif, as will be seen hereafter. Point symmetries, discussed in Section 2.1, always exist at a macroscopic scale and, in some cases, also at a microscopic scale, which means that they not only apply to the macroscopic properties but also put back all atoms in coincidence 


\section{Contribution of Symmetries in Condensed Matter}

with themselves. In other cases, point symmetries alone do not allow to obtain the perfect coincidence of the crystalline edifice with itself, and one has to combine them with fractional translations, that is, translations acting inside the unit cell. All these symmetries, point symmetries alone and point symmetries combined with a fractional translation, are called space group symmetries. The pure point symmetry is said symmorphic and the combined one non symmorphic. The space group symmetries, correctly positioned in the unit cell (they do not necessarily run through the same point) act inside the unit cell, and in particular inside the motif. Combined to the lattice translations, they make up the space group of the crystal. Thanks to them, the complete description of a crystal will now require the knowledge of the positions of much less atoms: only those contained in what is called the asymmetric unit. Applying all space group symmetries on the asymmetric unit will yield the motif, and then, applying the lattice translations on the latter will yield the entire crystal. The space groups thus describe the symmetry of the crystals' microscopic structure, constituting their "identity card". There exists in total 230 space groups, all gathered in the International Tables for Crystallography, Volume A [1]. Note that all the necessary information can also be found on the Bilbao Crystallographic Server [6].

We shall in this Section first introduce the two existing non symmorphic space group symmetries: glide planes and screw axes, that is a point symmetry (reflection and rotation, respectively) combined with a fractional translation (glide translation). Then we will briefly discuss how to obtain the 230 space groups, by combining the 14 Bravais lattices and the 32 point groups, adding or not glide translations. Last, we will explain how to read the "identity card" of a space group in the International Tables for Crystallography, from two selected examples.

Use will be made in subsections 2.3.1 and 3.2, and in other chapters (mainly those by Perez-Mato et al. and Tasci et al.) of the international notation [1]. A space group symmetry $\mathbb{W}$, more precisely, is represented as $\mathbb{W}=(W, w)$, where $W$ designates the point group symmetry and $w$ the additional fractional translation to be applied after the point group symmetry. The rotation part $W$ is a $(3 \times 3)$ matrix while the translation part $w$ is a $(3 \times 1)$ column matrix. To describe and combine together space group symmetries, it is very convenient to use the following augmented $(4 \times 4)$ matrix representation:

$$
\mathbb{W}=\left(\begin{array}{cc}
\boldsymbol{W} & \boldsymbol{w} \\
\boldsymbol{o} & 1
\end{array}\right)=\left(\begin{array}{cccc}
W_{11} & W_{12} & W_{13} & w_{1} \\
W_{21} & W_{22} & W_{23} & w_{2} \\
W_{31} & W_{32} & W_{33} & w_{3} \\
0 & 0 & 0 & 1
\end{array}\right)
$$

Space groups generated, apart from the lattice translations, only by symmetry elements $\mathbb{W}$ with $w_{1}=$ $w_{2}=w_{3}=0$, that is only by pure point symmetries, are called symmorphic space groups. Otherwise, they are called non symmorphic space groups.

Another notation, used in some textbooks and in the chapter by Rodríguez-Carvajal and Bourée, is to represent every element $g$ of a space group by its Seitz symbol: $g=\left\{\alpha \mid \vec{\tau}_{\alpha}+\vec{R}_{n}\right\}$, where $\alpha$ designates the point group symmetry, $\vec{\tau}_{\alpha}$ the additional fractional translation to be applied after the point group symmetry, and $\vec{R}_{n}$ a lattice translation.

\subsubsection{Glide planes and screw axes}

Crystal invariances, without a fixed point and different from a translation, can be formed by combination of orientation symmetry (a reflection or a rotation) and fractional translation symmetry (i.e. acting inside the unit cell). One thus obtains a glide plane or a screw axis. These are the only additional symmetries to further take into account. It is recalled that according to the Chasles' Theorem, every direct isometry of a 3-dimensional euclidean space is equivalent to a screw displacement, namely a translation along a line followed (or preceded) by a rotation about that line. Such symmetries must be compatible with the lattice translation symmetry. Moreover, after applying $n$ times such a space group symmetry, where $n$ is the order of the underlying point symmetry, one must recover a lattice translation. These two requirements 


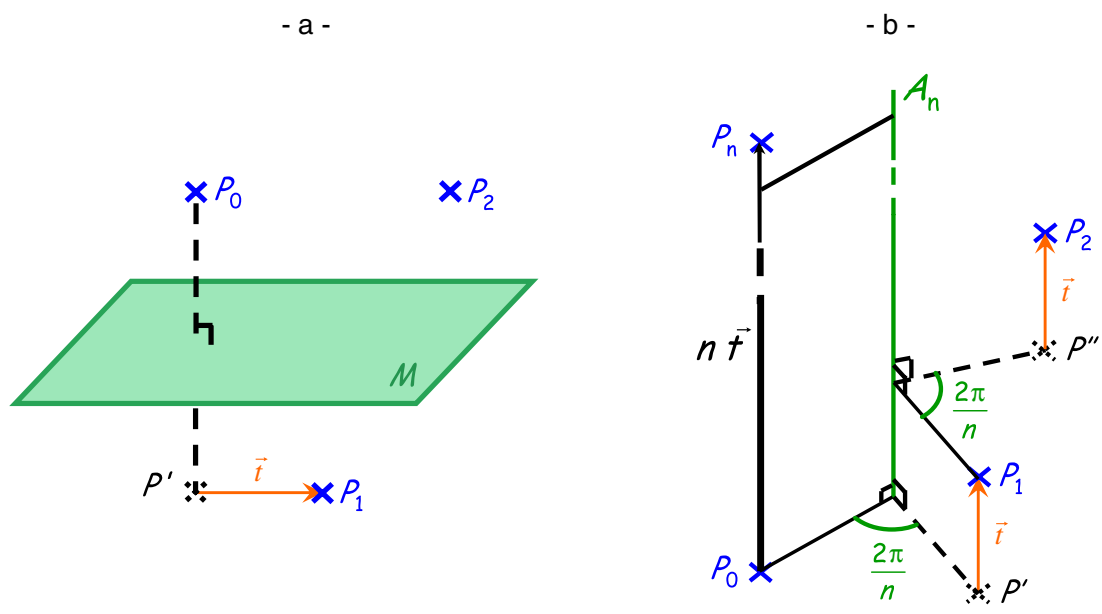

Figure 12. The two non symmorphic space group symmetries: a- glide plane and b- screw axis.

obviously will fix some constraints on the direction of the fractional translation, with respect to the orientation of the glide plane or the screw axis, and on the length of the fractional translation. The first constraint is that the fractional translation has to be parallel to a lattice plane or axis, to give a lattice translation by repeated occurrence. The second constraint will be discussed hereafter, by introducing more specifically the concerned space group symmetries.

- Glide planes

Let us consider a mirror plane and a translation vector $\vec{t}$ parallel to that plane, as plotted in figure 12-a. The glide plane symmetry, applied on a starting point $P_{0}$, generates point $P_{1}$ as follows: the reflection symmetry transforms point $P_{0}$ into the intermediate immaterial point $P^{\prime}$ and the translation symmetry then transforms point $P^{\prime}$ into point $P_{1}$. Applying this glide plane now on point $P_{1}$ yields point $P_{2}$. Since the reflection symmetry is of order 2, applying twice the glide plane must be equivalent to applying a lattice translation $\vec{T}$ (as defined in eq. (2.16)), so that one must have:

$$
\overrightarrow{P_{0} P_{2}}=2 \vec{t}=\vec{T}
$$

For example, if the glide translation is parallel to $\vec{c}, \vec{T}=w \vec{c}$ and eq. (2.20) yields $\vec{t}=\vec{c} / 2$ since $\vec{t}$ must act inside the unit cell (imposing $w=1)$. The augmented $(4 \times 4)$ matrix for such a so-called $c$ plane, perpendicular to the $a$-axis at $x=1 / 4$, for example, is written:

$$
\mathbb{W}_{\text {glide plane } c \text { at }\left(\frac{1}{4} y z\right)}=\left(\begin{array}{rrrr}
-1 & 0 & 0 & \frac{1}{2} \\
0 & 1 & 0 & 0 \\
0 & 0 & 1 & \frac{1}{2} \\
0 & 0 & 0 & 1
\end{array}\right)
$$

In the translation part of this matrix ( $4^{\text {th }}$ column), $w_{1}=1 / 2$ comes from the fact that the plane cuts the $a$-axis at $1 / 4$, while $w_{3}=1 / 2$ accounts for the glide translation. The condition that applying twice a glide plane must be equivalent to applying a lattice translation yields a limited number of possible glide translations and thus of glide plane types, listed in Table 5.

Let us comment these various symmetry planes and in particular give some precisions about their orientation, for a few of them. For a complete description, one should refer to the ITC, volume A, chapter 1 [1]. A mirror $m$ can be perpendicular to any primary direction and, when they exist, secondary and tertiary directions, except the secondary direction for the cubic crystal system (see Table 3). This 


\section{Contribution of Symmetries in Condensed Matter}

Table 5. Printed and graphical symbols for symmetry planes: mirror and glide planes (see text for further explanations). Note that the double glide plane $e$ exists only in centered cells.

\begin{tabular}{|c|c|c|c|c|}
\hline \multirow{2}{*}{$\begin{array}{l}\text { printed } \\
\text { symbol }\end{array}$} & \multirow{2}{*}{$\begin{array}{l}\text { symmetry } \\
\text { plane }\end{array}$} & \multicolumn{2}{|c|}{ graphical symbol } & \multirow{2}{*}{$\begin{array}{c}\text { nature of } \\
\text { glide translation }\end{array}$} \\
\hline & & $\begin{array}{c}\text { normal to } \\
\text { projection plane }\end{array}$ & $\begin{array}{c}\text { parallel to } \\
\text { projection plane }\end{array}$ & \\
\hline$m$ & $\begin{array}{c}\text { reflection plane } \\
\text { (mirror plane) }\end{array}$ & & & none \\
\hline$a, b, c$ & $\begin{array}{c}\text { 'axial' } \\
\text { glide plane }\end{array}$ & $\begin{array}{l}-\bar{t} \bar{t} / / \text { proj. plane } \\
\cdots \cdots \ldots . . . \cdots \cdots \\
\vec{t} \perp \text { proj. plane }\end{array}$ & & $\vec{a} / 2, \vec{b} / 2$, or $\vec{c} / 2$ respectively \\
\hline$e$ & $\begin{array}{l}\text { 'double' } \\
\text { glide plane }\end{array}$ & $\cdots-\cdots-\cdots-\cdot$ & & $\begin{array}{l}(\vec{a} / 2 \text { and } \vec{b} / 2),(\vec{b} / 2 \text { and } \vec{c} / 2) \text {, or } \\
(\vec{a} / 2 \text { and } \vec{c} / 2) ; \text { OR }((\vec{a} \pm \vec{b}) / 2 \text { and } \vec{c} / 2), \\
\text {... etc for tetragonal and cubic lattices }\end{array}$ \\
\hline$n$ & $\begin{array}{l}\text { 'diagonal' } \\
\text { glide plane }\end{array}$ & $-\cdot-\cdot-\cdot$ & $\swarrow$ & $\begin{array}{l}(\vec{a}+\vec{b}) / 2 \text { or }(\vec{b}+\vec{c}) / 2 \text { or }(\vec{a}+\vec{c}) / 2 ; \\
\text { OR }(\vec{a} \pm \vec{b} \pm \vec{c}) / 2 \text { for tetragonal } \\
\text { and cubic lattices }\end{array}$ \\
\hline$d$ & $\begin{array}{l}\text { 'diamond' } \\
\text { glide plane }\end{array}$ & - $\cdot \leftarrow-\cdot-$ & $\left|\frac{1}{8}\right| \frac{3}{8}$ & $\begin{array}{l}(\vec{a} \pm \vec{b}) / 4 \text { or }(\vec{b} \pm \vec{c}) / 4 \text { or }(\vec{c} \pm \vec{a}) / 4 \\
\text { OR }( \pm \vec{a} \pm \vec{b} \pm \vec{c}) / 4 \text { for tetragonal } \\
\text { and cubic lattices }\end{array}$ \\
\hline
\end{tabular}

statement is quite straightforward, looking at the symmetry of the various conventional cells. For glide planes, the possible orientations are of course a bit more restricted, due to the additional constraints discussed above. Except for the cubic system, a plane $a$ with $\vec{t}=\vec{a} / 2$ (and thus parallel to $\vec{a}$ ) can be perpendicular only to the $\vec{b}$ or $\vec{c}$ axis and a plane $b$ with $\vec{t}=\vec{b} / 2$ only to the $\vec{a}$ or $\vec{c}$ axis, while a plane $c$ with $\vec{t}=\vec{c} / 2$ can have more possible orientations since the rotation axis along $c$ is of higher order in tetragonal and hexagonal conventional cells ${ }^{6}$. A plane $c$, parallel to the $c$-axis, can thus be perpendicular to $\vec{a}$ or $\vec{b}$ but also to the diagonal of the $(a, b)$ face in the case of a tetragonal cell, for example. Last, for planes $e, n$ and $d$ involving at least two lattice vectors (see Table 5), it is straightforward to determine their orientation once the glide translation is known, and vice versa. As an example, for a $n$ plane, the glide translation is parallel to the diagonal of the face to which the plane is parallel, so that for a $n$ plane perpendicular to $\vec{c}$, the glide translation can only be $(\vec{a}+\vec{b}) / 2$, while for a $n$ plane perpendicular to [1 $1 \overline{1} 0], \vec{t}$ has to be $(\vec{a}+\vec{b}+\vec{c}) / 2$.

It is worth making a last comment about the double glide planes $e$, since they were recently added in the ITC [1] and replace some previously named $a, b$ and $c$ planes. This double glide plane is encountered only in centered cells, as illustrated hereafter. As an example, let us consider a space group of the $A$ orthorhombic Bravais lattice having a plane $b$ perpendicular to the $a$-axis. Due to the $A$-centering, the glide translation $\vec{t}=\vec{b} / 2$ of that glide plane yields the existence of the additional glide translation $\vec{t}^{\prime}=\vec{t}+(\vec{b}+\vec{c}) / 2=\vec{b}+\vec{c} / 2$ (modulo $\vec{b}$ ), that is, since $\vec{t}^{\prime}$ must act inside the unit cell, $\vec{t}^{\prime}=\vec{c} / 2$. This previously named $b$ plane is thus renamed $e$ plane, to indicate that it is both of types $b$ and $c$ (see Table 5). As a consequence, 5 space groups were renamed: No. 39 ( $A b m 2$ is now Aem 2), No. 41 (Aba2

\footnotetext{
${ }^{6}$ In a cubic cell described by the Laue class $m \overline{3} m$, the axes $\vec{a}, \vec{b}$ and $\vec{c}$ are all of higher degree of symmetry (fourfold axis), so that a plane $a$ can also be perpendicular to $\vec{b} \pm \vec{c}$ and a plane $b$ to $\vec{a} \pm \vec{c}$. In the same way, a plane $c$ can be perpendicular to $\vec{a}, \vec{b}$ or the diagonal of the $(a, b)$ face.
} 


\section{EPJ Web of Conferences}

is now Aea2), No. 64 ( $\mathrm{Cmca}$ is now Cmce), No. 67 (Cmma is now Cmme), and No. 68 (Ccca is now $\mathrm{Ccce}$ ) (see next subsection for the explanation of these notations and the space groups' numbering).

- Screw axes

Let us now consider a rotation axis of order $n(n=2,3,4$ or 6$)$ and a translation vector $\vec{t}$ parallel to that axis, as plotted in figure 12-b. The screw axis, applied on a starting point $P_{0}$, generates point $P_{1}$ as follows: the rotation symmetry transforms point $P_{0}$ into the intermediate immaterial point $P^{\prime}$ and the translation symmetry then transforms point $P^{\prime}$ into point $P_{1}$. Applying this screw axis symmetry a second time, that is on point $P_{1}$, yields point $P_{2}$, and so forth until point $P_{n}$, obtained from point $P_{0}$ after $n$ successive applications of the screw axis (total angle $n \times 2 \pi / n=2 \pi$ and total glide translation $n \vec{t}$ ). Applying $n$ times the screw axis must be equivalent to applying a lattice translation $\vec{T}$, so that one must have:

$$
\overrightarrow{P_{0} P_{n}}=n \vec{t}=\vec{T}
$$

For example, if the glide translation is parallel to $\vec{c}$, eq. (2.22) yields:

$$
n \vec{t}=p \vec{c} \Rightarrow \vec{t}=\frac{p}{n} \vec{c}
$$

with $p$ an integer strictly smaller than $n$, for the glide translation to act inside the unit cell, that is $p=0,1, \ldots, n-1$. The Hermann-Mauguin symbol for a pure rotation (i.e. for $p=0$ ) is $n$, as seen previously, while that for a screw axis (i.e. for $0<p<n$ ) is $n_{p}$.

As a result, for $n=2$, only the pure rotation 2 and the screw diad $2_{1}$ exist; for $n=3$, only the pure rotation 3 and screw triads $3_{1}$ and $3_{2}$ exist, etc ... As concerns their orientation, pure rotation axes as well as screw axes of order $n$ can be parallel to the primary direction, and/or, when they exist, to the secondary and/or tertiary ones, regarding their degree of symmetry (see Tables 1 and 3). For $n=3$, for example, they can exist only in the hexagonal cell and are thus parallel to [001] (primary direction) or in the cubic cell and are thus parallel to $\langle 111\rangle$ (secondary direction). The augmented $(4 \times 4)$ matrix for a screw axis $n_{p}$ parallel to the $c$-axis at $x=1 / 4$ and $y=0$, for example, is written:

$$
\mathbb{W}_{\text {screw axis } n_{p} \text { at }\left(\frac{1}{4} 0 z\right)}=\left(\begin{array}{cccc}
\cos \frac{2 \pi}{n} & -\sin \frac{2 \pi}{n} & 0 & \frac{1}{2} \\
\sin \frac{2 \pi}{n} & \cos \frac{2 \pi}{n} & 0 & 0 \\
0 & 0 & 1 & \frac{p}{n} \\
0 & 0 & 0 & 1
\end{array}\right)
$$

Let us now focus on $4_{p}$ screw axes. Such axes can be encountered either in the tetragonal system (parallel to [001], i.e. to the $c$-axis) or in the cubic one (parallel to $\langle 100\rangle$, i.e. to the $a, b$, and $c$ axes). Figure 13 gives a representation of the 4 possible axes of order 4 (except $\overline{4}$ ) in perspective (top figures) and viewed from the top (bottom figures). In each top figure, points 2, 3, 4 and 5 are obtained by applying one, two, three and four times, respectively, the considered symmetry on point 1 . Considering the symmetry axis parallel to the $c$-axis, all points lie at $z=0$ for the 4 -axis, while for a $4_{p}$-axis $(p=1,2,3)$, point 2 is lying at $z=p / 4$, point 3 at $z=2 p / 4$, etc ... For $p=2$ and 3 , points 3,4 and 5 are lying at $z \geq 1$ so that they are outside of the initial unit cell. One can thus apply the lattice translation $-\vec{c}$ or $-2 \vec{c}$ on these points, yielding points $3^{\prime}, 4^{\prime}$ and 5' with $0 \leq z \leq 1$. Looking from the top, one sees the various schemes plotted schematically below each perspective figure, which explains the symbol used to represent each of these $4_{p}$ axes (see Table 6). As an example, for the $4_{1}$-axis, $z$ increases at each $\pi / 2$ rotation. In the bottom figure, this is represented by the dashes in the square's corners, indicating the direction of rotation when going up along the $z$ direction.

Table 6 lists all existing rotations, rotoinversions and screw axes, with the following information: their printed and graphical symbol, their name, and the glide translation, when it exists, given in units of the shortest lattice translation vector parallel to the axis. 


\section{Contribution of Symmetries in Condensed Matter}
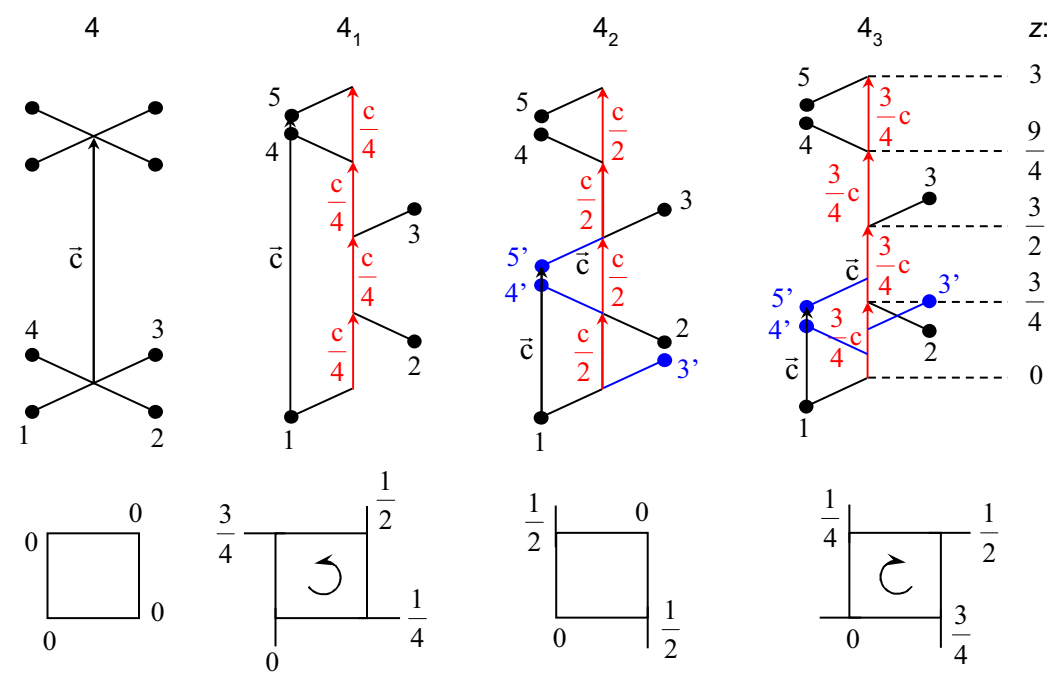

Figure 13. The fourfold rotation and screw axes, plotted in perspective parallel to the $c$-axis (top figures) and viewed from the top (bottom figures) in order to evidence their graphical symbol: pure rotation 4, screw axis $4_{1}$ (glide translation $\vec{t}=\vec{c} / 4$ ), screw axis $4_{2}$ (glide translation $\vec{t}=\vec{c} / 2$ ), and screw axis $4_{3}$ (glide translation $\vec{t}=3 \vec{c} / 4$ ). See text for further explanations.

\subsubsection{How to obtain and name the 230 space groups}

Combining the 14 Bravais lattices with the 32 crystallographic point groups (or crystal classes), one obtains in total 230 space groups. They are all listed in Tables 7 and 8 , by increasing number (No.), that is in the same order than in the ITC [1], using the short international (Hermann-Mauguin) symbols. These numbers correspond to an international numbering of the space groups, for an easier locating of a given space group among the 230 ones. In this list, the conventional cell and the crystal class to which the space group belongs are also indicated. Before explaining briefly how to obtain all space groups, let us first explain their symbol.

As for point groups, both Hermann-Mauguin (short and full) and Schoenflies symbols exist for space groups. But in crystallography, the former is used because the symmetry elements appear explicitly in the Hermann-Mauguin symbol, so that it makes it much more convenient. These symbols will thus be presented in detail. Both the short and the full international (Hermann-Mauguin) symbols consist of two parts: $\mathrm{i}$ - a letter indicating the lattice type, $P, I, F, A, B, C$ or $R$ (see subsection 2.2.1 and Table 4), ii- a set of one, two or three characters, indicating the space group symmetry elements present along the primary, secondary and tertiary directions (when they exist), respectively, as for point groups (see Table 3). Here again, the direction of a symmetry axis is given by the direction of the axis, while that of a symmetry plane is given by its normal. If various types of symmetry planes exist along the same direction, only one symbol will be given, following this order of descending priority: $m, e, a, b, c, n, d$ (see Table 5), while if both pure rotations and screw axes exist, the pure rotations will be given (except for a few exceptions, see Ref. [1]). In the full symbol, when both symmetry axes and planes are present along a given direction, the two characters are given, separated by a slash, indicating that the plane is perpendicular to the axis. In the short symbol, symmetry axes are suppressed as much as possible: only the symmetry plane's symbol is given when the plane is perpendicular to an axis of order 2, for orthorhombic, tetragonal, hexagonal and cubic cells, or when the plane is perpendicular to an axis of order 4 for the cubic cell (but not for the tetragonal one). As an example, space group I $4_{1} /$ amd (short symbol) belongs to the tetragonal system since an axis of order 4 exists along the primary direction but no axis of order 3 exists along the secondary direction. In this space group, the tetragonal cell is 


\section{EPJ Web of Conferences}

Table 6. Printed and graphical symbols for symmetry axes: rotation, rotoinversion and screw axes. The glide translation is given in units of the shortest lattice translation vector parallel to the axis. Axes of order 2 can be parallel or perpendicular to the projection plane, so that both representations are given, while those of order 3,4 and 6 are always perpendicular to the projection plane, that is parallel to the $c$-axis, except in the cubic system where axes of order 3 are inclined (the same symbol is used) and axes of order 4 are parallel and perpendicular to the projection plane (see Ref. [1] for their symbol when parallel to the paper).

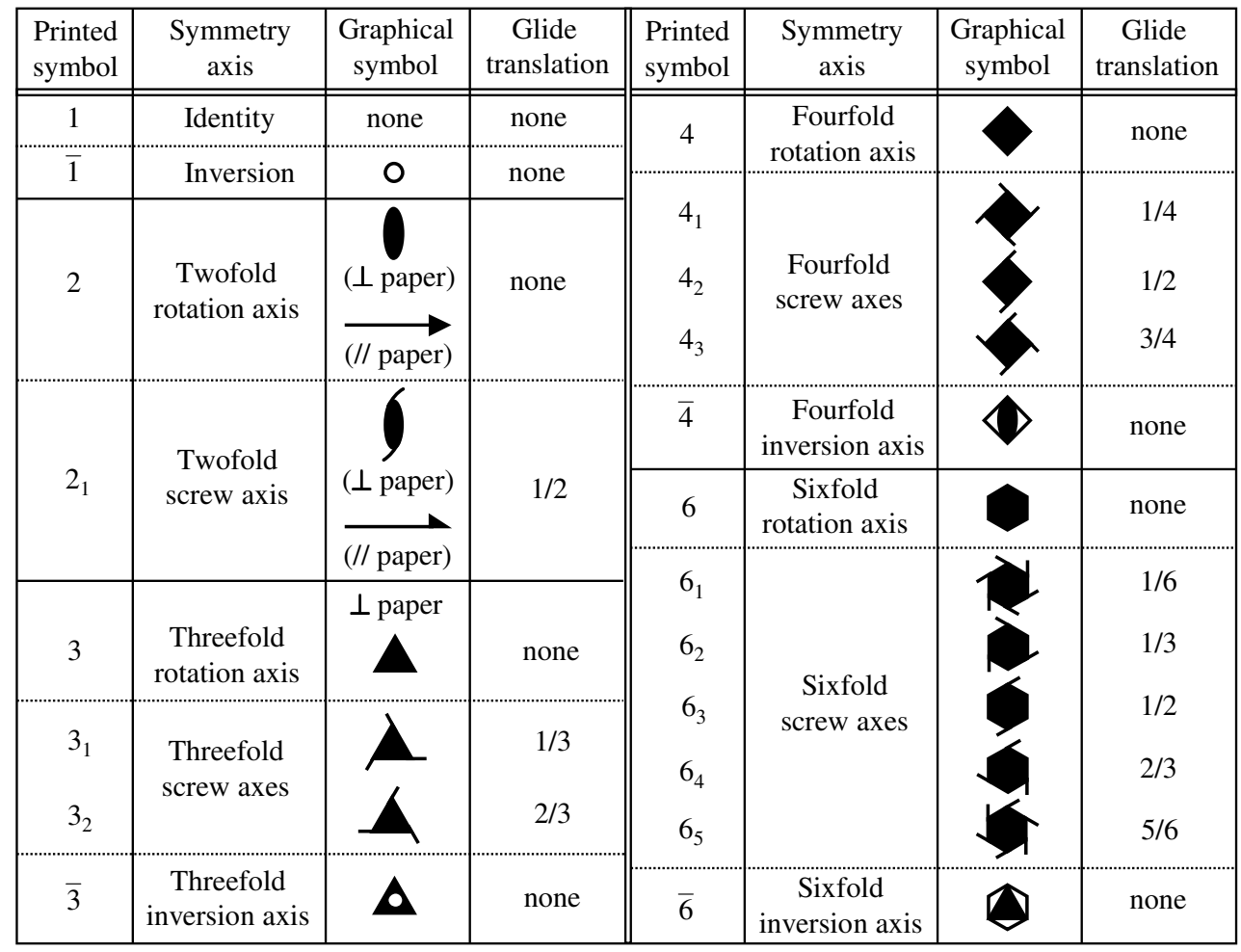

body-centered, a fourfold screw axis $4_{1}$ is parallel to the primary direction ([001]) and a glide plane $a$ is perpendicular to it, a mirror $m$ is perpendicular to the secondary direction ([100] and [010]) and a diagonal glide plane $d$ to the tertiary direction ([110] and [1 $\overline{10}]$ ). Due to the body-centering, additional planes and axes are present along each direction, but, following the priority rule explained above, only these $a, m$ and $d$ planes are given in the space group symbol. For example, $n$ planes are also present along the secondary direction, but the $m$ mirrors are given. The full symbol for that space group is $I 4_{1} / a 2 / m 2 / d$ indicating that twofold rotation axes are also present along the secondary and tertiary directions. As concerns space groups' Schoenflies symbols, the symbol of the corresponding point group is used with an additional subscript number. As an example, three space groups correspond to point group 2 (international symbol) or $C_{2}$ (Schoenflies symbol): these are $P 2, P 2{ }_{1}$, and $C 2$ (international symbol) or $C_{2}^{1}, C_{2}^{2}$, and $C_{2}^{3}$ (Schoenflies symbol).

Let us now briefly discuss how the 230 space groups can be obtained. One considers the 7 crystal systems, one after the other, by increasing degree of symmetry and one associates to each of them the compatible crystal classes, in the same order than listed in Table 1, and the compatible lattice types (see Table 4) starting with the $P$ type then with the centered ones in the order $C, A, F, I$ (for non hexagonal cells) or $R$ (for the hexagonal cell). In a first step, one considers for each couple (Bravais lattice, point group) the symmorphic operations, that is with no glide translation. All symmetry elements thus go through the center of the cell, and, for symmetry reasons, are duplicated at the corners. For each crystal system, one can thus built at least $n n^{\prime}$ symmorphic space groups, with $n$ the number of compatible 


\section{Contribution of Symmetries in Condensed Matter}

Table 7. The 230 space groups listed by increasing number (first part).

\begin{tabular}{|c|c|c|c|}
\hline \multirow[t]{2}{*}{ cell } & \multirow{2}{*}{$\begin{array}{c}\text { point } \\
\text { group }\end{array}$} & \multicolumn{2}{|c|}{ space group } \\
\hline & & No. & symbol \\
\hline \multirow[t]{2}{*}{$a$} & 1 & 1 & $P 1$ \\
\hline & $\overline{1}$ & 2 & $P \overline{1}$ \\
\hline \multirow[t]{13}{*}{$m$} & 2 & 3 & $P 2$ \\
\hline & & 4 & $P 2_{1}$ \\
\hline & & 5 & $C 2$ \\
\hline & $m$ & 6 & $P m$ \\
\hline & & 7 & $P c$ \\
\hline & & 8 & $\mathrm{Cm}$ \\
\hline & & 9 & $C c$ \\
\hline & $2 / m$ & 10 & $P 2 / m$ \\
\hline & & 11 & $P 2_{1} / m$ \\
\hline & & 12 & $C 2 / m$ \\
\hline & & 13 & $P 2 / c$ \\
\hline & & 14 & $P 2_{1} / c$ \\
\hline & & 15 & $C 2 / c$ \\
\hline \multirow[t]{35}{*}{ o } & 222 & 16 & $P 222$ \\
\hline & & 17 & $P 222_{1}$ \\
\hline & & 18 & $P 2{ }_{1}{ }_{1} 2$ \\
\hline & & 19 & $P 2_{1} 2_{1} 2_{1}$ \\
\hline & & 20 & $C 222_{1}$ \\
\hline & & 21 & $C 222$ \\
\hline & & 22 & $F 222$ \\
\hline & & 23 & $I 222$ \\
\hline & & 24 & $I 2_{1} 2_{1} 2_{1}$ \\
\hline & $m m 2$ & 25 & $P m m 2$ \\
\hline & & 26 & $P m c 2_{1}$ \\
\hline & & 27 & $P c c 2$ \\
\hline & & 28 & Pma2 \\
\hline & & 29 & $\mathrm{Pca}_{1}$ \\
\hline & & 30 & Pnc2 \\
\hline & & 31 & $P m n 2_{1}$ \\
\hline & & 32 & $\mathrm{Pba2}_{1}$ \\
\hline & & 33 & Pna $_{1}$ \\
\hline & & 34 & Pnn2 \\
\hline & & 35 & Cmm2 \\
\hline & & 36 & $C m c 2_{1}$ \\
\hline & & 37 & $C c c 2$ \\
\hline & & 38 & Amm2 \\
\hline & & 39 & $A b m 2$ \\
\hline & & 40 & Ama2 \\
\hline & & 41 & $A b a 2$ \\
\hline & & 42 & Fmm2 \\
\hline & & 43 & $F d d 2$ \\
\hline & & 44 & $\operatorname{Imm} 2$ \\
\hline & & 45 & $I b a 2$ \\
\hline & & 46 & $\operatorname{Ima} 2$ \\
\hline & $m m m$ & 47 & Pmmm \\
\hline & & 48 & Pnnn \\
\hline & & 49 & Pccm \\
\hline & & 50 & Pban \\
\hline
\end{tabular}
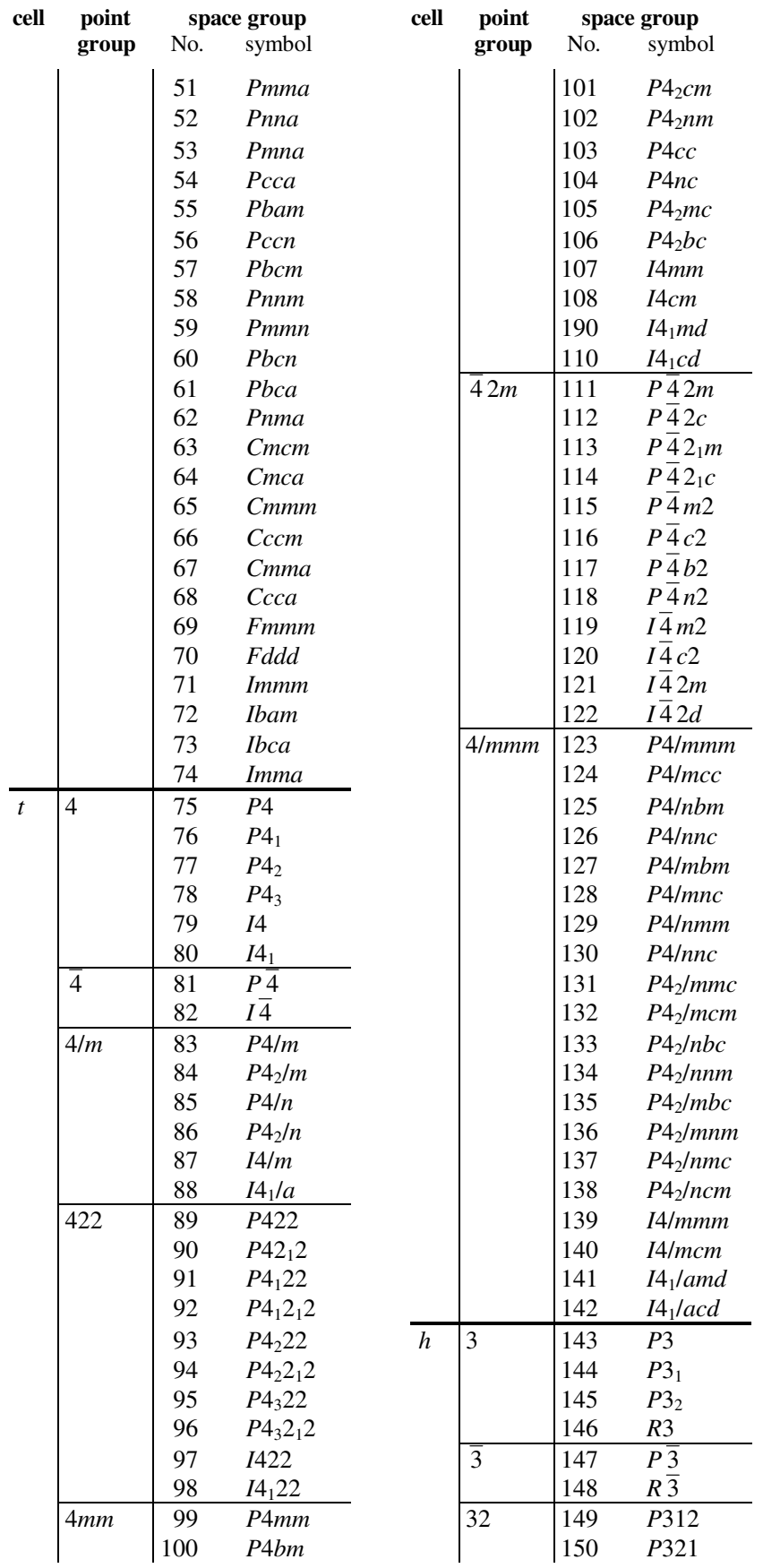

lattice types and $n^{\prime}$ the number of compatible crystal classes. In a second step, one adds for each couple (Bravais lattice, point group) some glide translation to each of the symmetry elements of the point group, one after the other, then to two of them and so forth. Doing so allows to obtain non symmorphic space groups. Only the space groups that are different from those previously obtained are kept. Contrary to symmorphic space groups, the symmetry elements do not all go through the same point. 


\section{EPJ Web of Conferences}

Table 8. The 230 space groups listed by increasing number (second part).
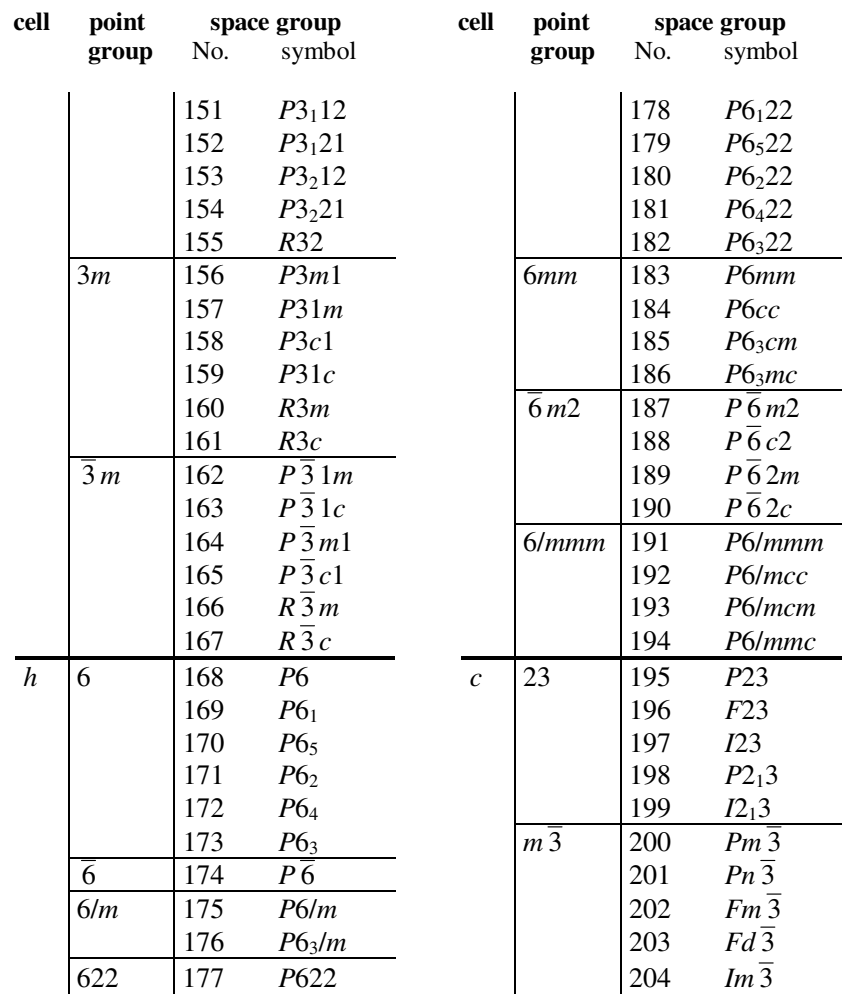

\begin{tabular}{|c|c|c|c|}
\hline \multirow{4}{*}{\multicolumn{2}{|c|}{ cell }} & \multicolumn{2}{|c|}{ space group } \\
\hline & & No. & symbol \\
\hline & & 205 & $\operatorname{Pa} \overline{3}$ \\
\hline & & 206 & $\operatorname{Ia} \overline{3}$ \\
\hline & \multirow[t]{8}{*}{432} & 207 & $P 432$ \\
\hline & & 208 & $P 4_{2} 32$ \\
\hline & & 209 & $F 432$ \\
\hline & & 210 & $F 4_{1} 32$ \\
\hline & & 211 & $I 432$ \\
\hline & & 212 & $\mathrm{P}_{3} 32$ \\
\hline & & 213 & $P 4,32$ \\
\hline & & 214 & $I 4_{1} 32$ \\
\hline & \multirow[t]{6}{*}{$\overline{4} 3 m$} & 215 & $P \overline{4} 3 m$ \\
\hline & & 216 & $F \overline{4} 3 m$ \\
\hline & & 217 & $I \overline{4} 3 m$ \\
\hline & & 218 & $P \overline{4} 3 n$ \\
\hline & & 219 & $F \overline{4} 3 c$ \\
\hline & & 220 & $I \overline{4} 3 d$ \\
\hline & \multirow[t]{9}{*}{$m \overline{3} m$} & 221 & $P m \overline{3} m$ \\
\hline & & 222 & $\operatorname{Pn} \overline{3} n$ \\
\hline & & 223 & $\operatorname{Pm} \overline{3} n$ \\
\hline & & 224 & $\operatorname{Pn} \overline{3} m$ \\
\hline & & 225 & $F m \overline{3} m$ \\
\hline & & 226 & $F m \overline{3} c$ \\
\hline & & 227 & $F d \overline{3} m$ \\
\hline & & 228 & $F d \overline{3} c$ \\
\hline & & 229 & $\operatorname{Im} \overline{3} m$ \\
\hline & & 230 & $\operatorname{Ia} \overline{3} d$ \\
\hline
\end{tabular}

As an illustration, let us consider all space groups that can be built from the monoclinic conventional cell, choosing $b$ for the unique axis. $n=2$ Bravais lattices exist, $m P$ and $m C$, and $n^{\prime}=3$ crystal classes are compatible with this cell, $2, m$ and $2 / m$. Six symmorphic space groups can first be obtained (see Table 7): $P 2, C 2, P m, C m, P 2 / m$, and $C 2 / m$ (No. 3, 5, 6, 8, 10 and 12, respectively). Then, the 2-axis can be replaced by a 2 -axis in space groups No. $3,5,10$ and 12 , yielding only two new space groups $P 2_{1}$ and $P 2_{1} / m$ (No. 4 and 11). Then, the $m$ mirror can be replaced by a $c$ plane in space groups No. 6, 8, 10 and 12 yielding four new space groups $P c, C c, P 2 / c$, and $C 2 / c$ (No. 7, 9, 13 and 15, respectively). Last, a glide translation is added for both the 2-axis and the $m$ mirror in space group No. 10 yielding $P 2_{1} / c$ (No. 14), while doing that in space group No. 12 does not yield any new space group. In total, 13 monoclinic space groups are thus obtained, among which exactly $n n^{\prime}=6$ symmorphic ones. But in some cases, more than $n n^{\prime}$ symmorphic space groups can be obtained. For example, Amm2 (space group No. 38, equivalent to $C 2 \mathrm{~mm}$, by interchanging the two axes $a$ and $c$, and to $C m 2 m$, by cyclic permutation of the three axes $a \rightarrow c, b \rightarrow a, c \rightarrow b$ ) and $C m m 2$ (space group No. 35) correspond to two different space groups (though built from the same Bravais lattice, since $A$ and $C$ correspond to the same lattice type, and the same point group). Indeed, two different orderings of the space groups symmetry elements along the primary, secondary and tertiary directions can yield different space groups. In $A m m 2$ space group, the twofold axis is parallel to the centered face while in the $C m m 2$ space group, it is perpendicular. As a second illustration, see space groups $P 6 / \mathrm{mcm}$ and $P 6 / \mathrm{mmc}$ in Table 8 .

\subsubsection{The International Tables for Crystallography}

It is useful to know how to interpret the information provided in the International Tables for Crystallography [1] for all space groups. To illustrate this, two selected examples have been 


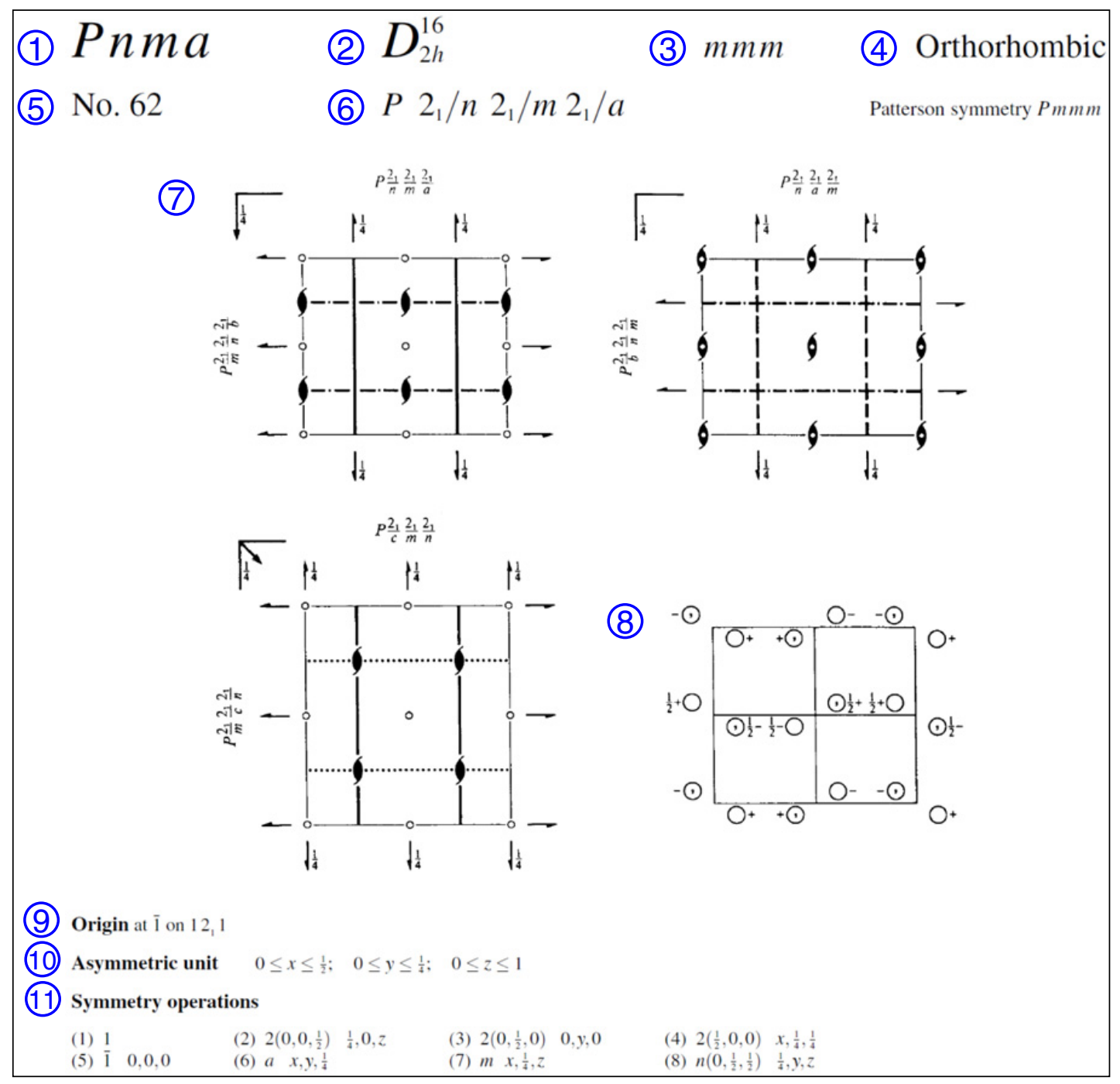

Figure 14. (Color online) First page of Pnma space group taken from the International Tables For Crystallography, Volume A (page 298) [1], with circled numbers added in blue (see text).

chosen: Pnma and $I 4 \mathrm{~mm}$. Only some specific points will be explained here. For a more complete description, one should refer to Ref. [1].

Figures 14 and 15 are reproductions of the information provided in the ITC for space group Pnma, on which blue circled numbers and a blue box were added for an easier legibility. As concerns the second page (figure 15), only the top part has been reproduced here. In the bottom part, information about group-subgroup relation is given: maximal isomorphic and non-isomorphic subgroups, as well as minimal non-isomorphic supergroups; but it will not be discussed here (see chapter by Perez-Mato et al.).

(1) and (6) are respectively the short and full international (Hermann-Mauguin) symbols of the considered space group, (2) is its Schoenflies symbol, (3) its point group in short international symbol, (4) its crystal system, and (5) its number. From all this information, one learns that this space group is non symmorphic, belongs to the orthorhombic primitive $(P)$ Bravais lattice, and has the following symmetry elements: planes $n \perp \vec{a}, m \perp \vec{b}$, and $a \perp \vec{c}$, and screw axes $2_{1}$ perpendicular to each of them, 


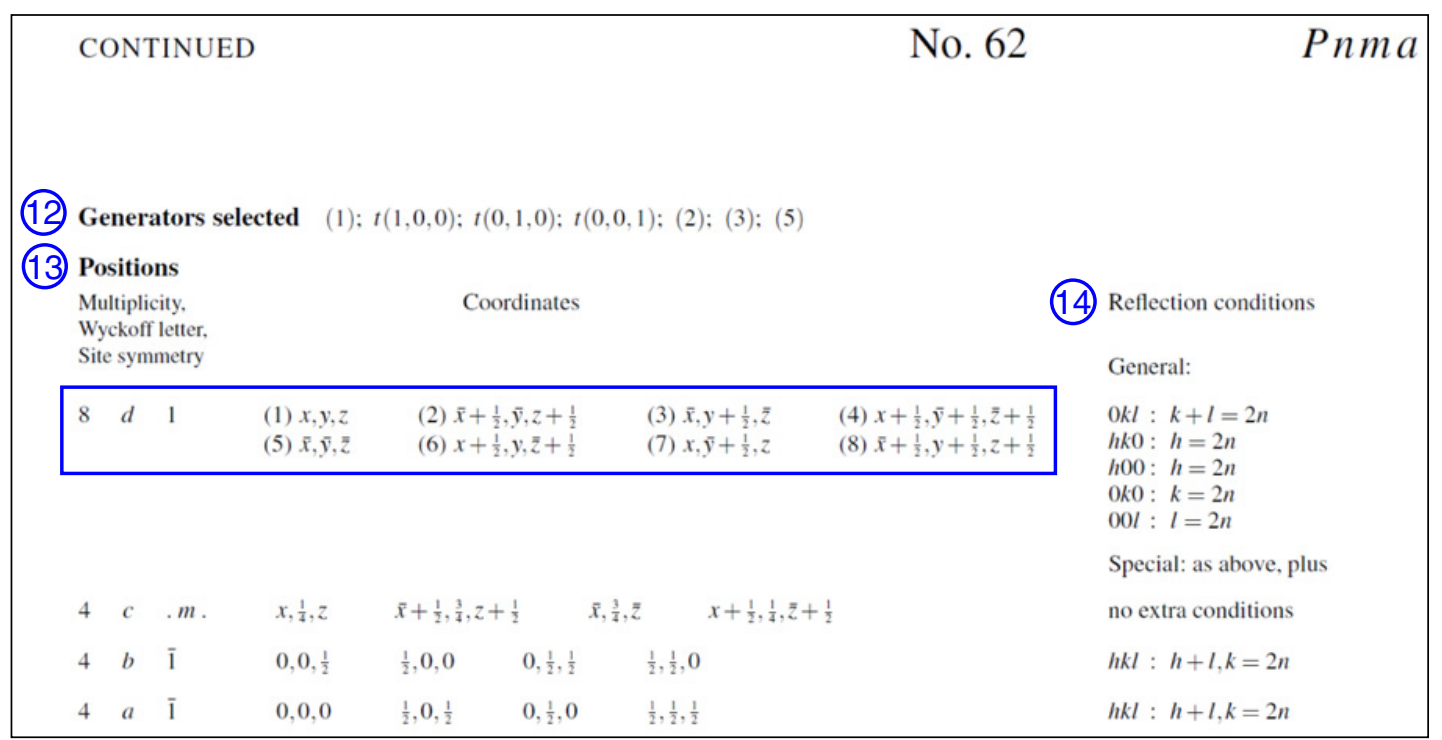

Figure 15. (Color online) Top part of the second page of Pnma space group taken from the International Tables For Crystallography, Volume A (page 299) [1], with circled numbers and a box added in blue (see text).

that is $\| \vec{a}, \vec{b}$, and $\vec{c}$. Suppressing in the international symbol the lattice type and the glide translation from the various space group operations yields the international symbol for the point group: planes $n, m$ and $a$ become $m$ mirrors and screw axes $2_{1}$ become rotation axes 2, yielding $2 / m 2 / m 2 / m$ point group, in full notation, that is $\mathrm{mmm}$ in short notation.

The three diagrams labeled (7) are projections in the $(a, b)$ plane of all symmetry operations that exist in the unit cell (using the graphical symbols given in Tables 5 and 6), with the following convention: $\vec{a}$ points to the bottom, $\vec{b}$ points to the right ( $\vec{c}$ is thus perpendicular to the paper pointing forwards). Since there are six different manners to name $a, b$ and $c$ in an orthorhombic cell, this yields six different international symbols and six different diagrams for the same space group. This information is given looking at the three diagrams in the 'normal way' (the top left one corresponds to the same setting as the symbol given in (1)) and then after rotating clockwise the paper by $90^{\circ}$ degrees. For symmetry elements parallel to the $(a, b)$ plane at $z \neq 0$, the spot height $z$ is written next to the graphical symbol. Along $c$ for example, one can clearly see in the top left diagram the presence of $2_{1}$ axes at $\left(x_{0}, y_{0}\right)$ positions with $x_{0}=1 / 4$ and 3/4, $y_{0}=0,1 / 2$ and 1 and the presence of a plane $a$ (since the arrow in its graphical symbol points in the $a$ direction) at $z=1 / 4$. Note also the presence of the inversion (not explicitly written in the international symbol, but whose presence can be guessed from the existence of planes perpendicular to the twofold axes or from the name of the point group which is centrosymmetrical). A last comment deserves to be made: for symmetry reasons, if a symmetry element is present at $\alpha=0$ (where $\alpha=x, y$ or $z$ ), it is also present at $\alpha=1 / 2$ and 1 , while if it is present at $\alpha=1 / 4$, it is also present at $\alpha=3 / 4$.

The diagram labeled (8) is the same projection as the top left diagram labeled (7) (that is for the standard setting of this space group, Pnma) except that the equivalent atomic positions are plotted instead of the symmetry operations. Starting from an atom at some arbitrary position $(x, y, z)$ inside the unit cell close to the origin and applying all symmetry elements (inversion, symmetry planes and axes, and lattice translations) yields all the equivalent positions plotted in the figure: note that all positions inside the unit cell and in its immediate surrounding are plotted. Points represented by $\bigcirc$ and $\bigcirc$ are related by inversion, rotoinversion or mirror symmetry. Last, the spot height is also indicated next to each atom symbol as follows: the symbols + and - stand for $+z$ and $-z$, while the symbols $\frac{1}{2}+$ and $\frac{1}{2}-$ 


\section{Contribution of Symmetries in Condensed Matter}

stand for $\frac{1}{2}+z$ and $\frac{1}{2}-z$, respectively. Last, it is worthwhile mentioning that the number of equivalent positions inside the unit cell corresponds to the order of the point group (since the cell is primitive) or, equivalently, to the number of symmetry operations (since all of them are of order 2): these 8 symmetry elements are identity, inversion, planes $n, m$ and $a$ and screws axes $2_{1}, 2_{1}$ and $2_{1}$ along $a, b$ and $c$, respectively.

Since the choice of the origin is arbitrary, (9) tells on which symmetry elements it was chosen here: on the inversion center located on the $2_{1}$ axis parallel to $\vec{b}$. (10) gives the volume occupied by the asymmetric unit: the knowledge of the atoms contained in this volume only is sufficient to reconstruct the entire crystal (applying the symmetry planes and axes first, to obtain the motif, then the lattice translations). All space group operations except lattice translations are listed in (11) with the following items: a number (between parenthesis), the nature of the symmetry and its position. For example, operation number (2) is a twofold screw axis with the glide translation along the $c$ axis and located at $1 / 40 z$; operation number (8) is a glide plane $n$ with glide translation $(\vec{b}+\vec{c}) / 2$ and located at $1 / 4 y z$. Note that the list contains 8 different symmetry operations, as already mentioned, but not all of them are necessary to generate the space group. An arbitrary choice of generators is then given in (12): symmetry operations numbers (1), (2), (3), and (5), together with the lattice translations. This list is very useful for softwares since it allows to generate entirely the space group symmetries with a reduced number of symmetry elements. (13) gives the list of Wyckoff positions for the considered space group: the general one (in the upper block, boxed in blue) and the special ones (in the blocks below, by increasing degree of symmetry). For each Wyckoff position, the four following items are provided, from left to right: i- the multiplicity $M$ of the site, that is the number of equivalent positions in the unit cell, ii- the Wyckoff letter of the site, starting with $a$ at the bottom position (the most symmetrical one and thus that with the smallest multiplicity $M$ ) and continuing upwards in alphabetical order, iii- the site symmetry, iv- the list of coordinates (modulo 1) for all equivalent positions obtained by applying symmetry operations (1) to (8) (see (11) to an initial atom. As concerns the general position, the initial atom is chosen at an arbitrary position $(x, y, z)$ and the numbering of the atoms, given between parentheses, is the same as the one for the symmetry operations in (11). Note also that the multiplicity of this site (number of coordinates) is equal to the number of symmetry operations given in (11), that is to the product of the point group's order by the multiplicity of the cell. A Wyckoff site is named by the two first items: site ' $8 d$ ', for example. The general Wyckoff site ' $8 d$ ' has a multiplicity $M=8$, as already known from the information given in the previous page (diagram (8) and list of symmetry operations (11)) and is located anywhere (on identity ' 1 '), since it is general. If one now considers an atom located on the mirror $m$ perpendicular to $\vec{b}$ at $y=1 / 4$, that is on symmetry element denoted '. $m$.', the multiplicity is now divided by two (site ' $4 c$ '): for example, positions (1) and (7) are now the same one, $(x, 1 / 4, z)$. Last, (14) gives the list of extinction rules, which will be explained when presenting the reciprocal space's properties (see section 2.4.2).

As an example, let us consider the structure of $\mathrm{LaMnO}_{3}$, crystallizing in the Pbnm space group (cf. J. Rodríguez-Carvajal, M. Hennion, F. Moussa, A. H. Moudden, L. Pinsard, and A. Revcolevschi, Phys. Rev. B 57, R3189 (1998)). As can be seen from Fig. 14 and from Table 4.3.2.1 in the ITC, Volume A [1], this space group is equivalent to Pnma by doing the following permutation: $a \rightarrow b, b \rightarrow c$, and $c \rightarrow a$. The asymmetric unit, using this standard setting Pnma, is:

$\begin{array}{llll} & x & y & z \\ \mathrm{La} & 0.0490 & 0.25 & -0.0078 \\ \mathrm{Mn} & 0 & 0 & 0.5 \\ \mathrm{O} 1 & 0.4874 & 0.25 & 0.0745 \\ \mathrm{O} 2 & 0.3066 & 0.0384 & 0.7256\end{array}$

Looking at Fig. 14, one immediately notices that La and $\mathrm{O} 1$ atoms are located on the $4 c$ Wyckoff site, $\mathrm{Mn}$ on the $4 b$ site and $\mathrm{O} 2$ on the $8 d$ site, yielding the motif $\mathrm{La}_{4} \mathrm{Mn}_{4} \mathrm{O}_{12}$. So once the space group is known as well as the Wyckoff sites the atoms occupy, only 7 coordinates out of $60(3 \times 20$ atoms in the unit cell) have to be determined in this example in order to completely determine the crystal structure. 


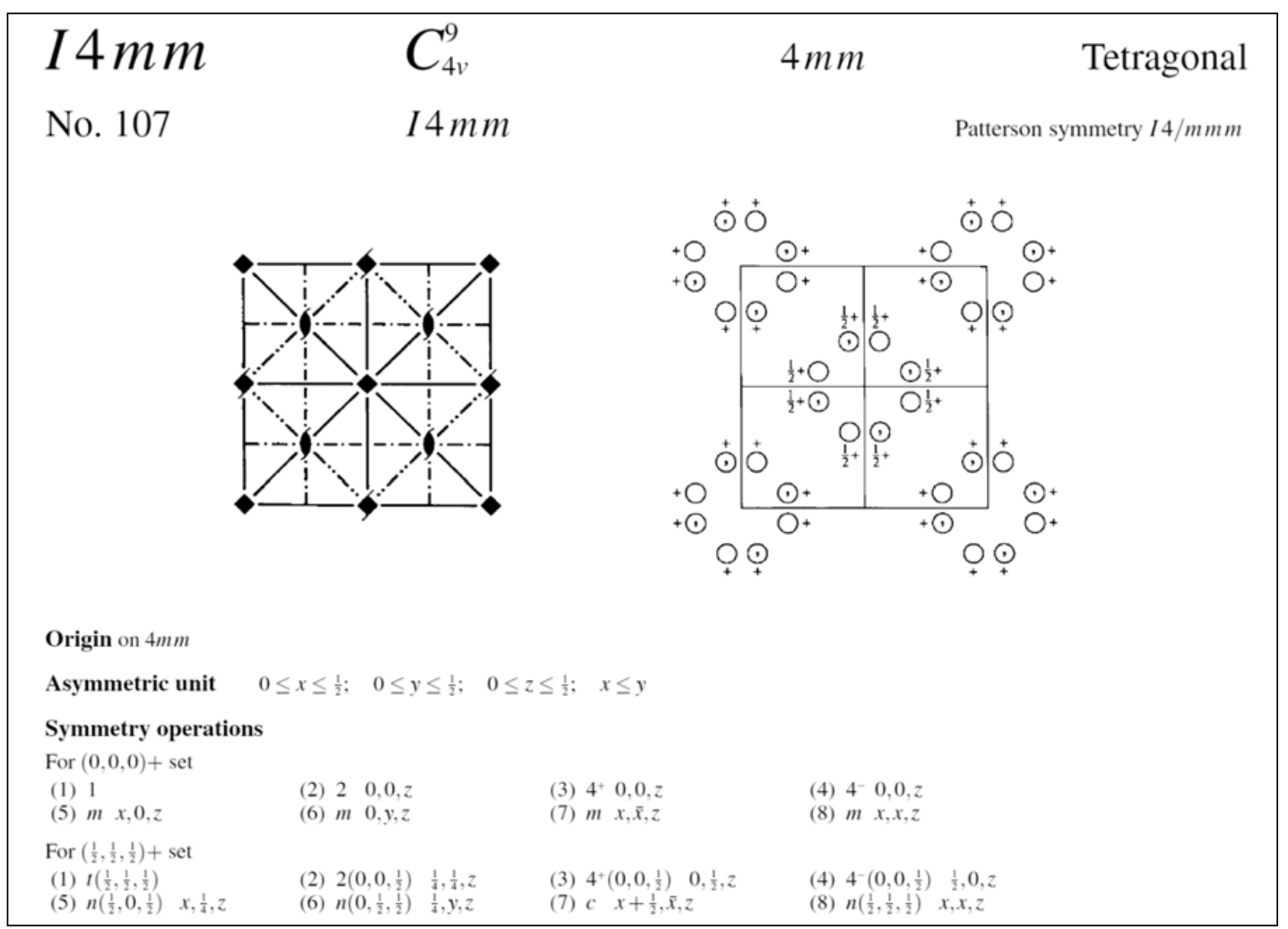

Figure 16. First page of $I 4 \mathrm{~mm}$ space group taken from the International Tables For Crystallography, Volume A (page 398) [1].

Figures 16 and 17 are reproductions of the information provided in the ITC for space group $I 4 \mathrm{~mm}$. Here again, only the top part of the second page has been reproduced in figure 17. In this symmorphic body-centered tetragonal space group, a fourfold rotation axis is along $c$ (primary direction) and mirrors $m$ are perpendicular to $\langle 100\rangle$ (secondary direction) and to $\langle 110\rangle$ (tertiary direction). Only two specific items will be focused on, because of their particularities as compared to the previously discussed space group. The first difference is the presence here of a symmetry operation of order strictly larger than 2: the fourfold axis. The latter is thus decomposed in three different symmetry operations in the list of symmetry operators given in the first page (see operations (3), (2) and (4) in Fig. 16), each of them generating only one new position: they correspond, respectively, to consecutive applications of the 4 axis, a first time anti-clockwise $\left(2 \pi / 4\right.$, labeled $\left.4^{+}\right)$, a second time $(\pi$, labeled 2$)$, and a third time anticlockwise, that is once clockwise $\left(3 \pi / 4 \equiv-\pi / 4\right.$, labeled $\left.4^{-}\right)$. The advantage of doing so is that here again, the total number of symmetry operations is the same as the general Wyckoff site's multiplicity $M$, as will be seen hereafter. The second difference is the non primitive type of the cell, which has various implications. First, the symmetry operations are decomposed in two sets due to the multiplicity $m=2$ of the unit cell: in the $(0,0,0)+$ set are listed the height symmetry operations of the space group, without taking into account the body-centering of the cell (identity, axis 4 decomposed in three parts, and the four mirrors), applying the $I$-centering on these 8 symmetry operations generates the 8 other symmetry operations listed in the set $\left(\frac{1}{2}, \frac{1}{2}, \frac{1}{2}\right)+$. In total, this space group thus contains 16 symmetry operations: 2 (multiplicity of the cell) $\times 8$ (point group's order). Second, the translation $t\left(\frac{1}{2}, \frac{1}{2}, \frac{1}{2}\right)$ is of course added in the list of selected generators (see Fig. 17). Last, only half of the coordinates are explicitly listed for all Wyckoff positions and the centering translations $(0,0,0)+$ and $\left(\frac{1}{2}, \frac{1}{2}, \frac{1}{2}\right)+$ are written above: the 


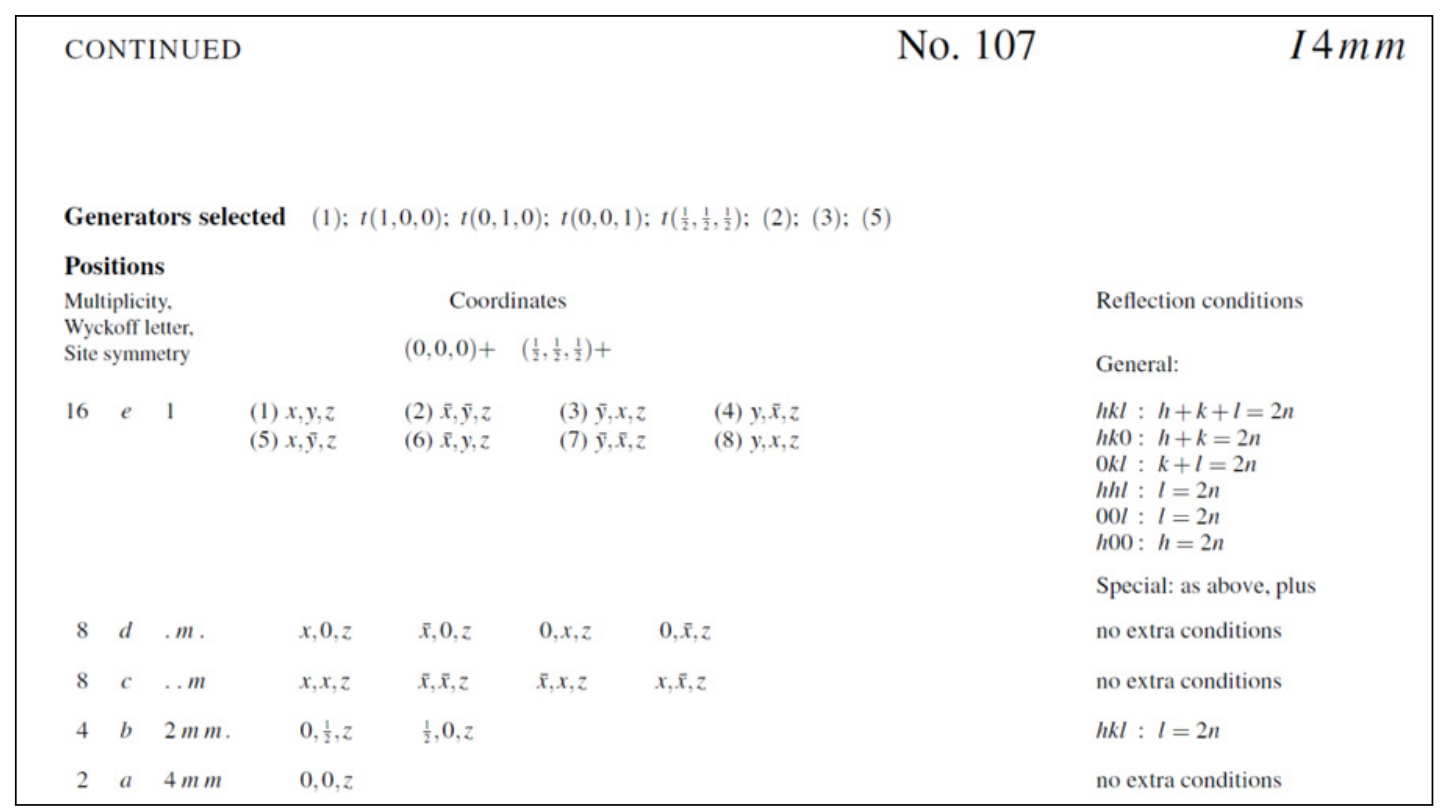

Figure 17. Top part of the second page of $I 4 \mathrm{~mm} 2$ space group taken from the International Tables For Crystallography, Volume A (page 399) [1].

' + ' sign indicates that, in order to obtain the complete Wyckoff position, one must add the components of these translations to the listed coordinates. More generally, for a Wyckoff site of multiplicity $M$ in a centered cell of multiplicity $m$, only $M / m$ coordinates are explicitly listed. Note that the numbers given between parentheses for the coordinates of the general site correspond to those of the symmetry operations given in the set $(0,0,0)+$ (see Fig. 16).

\subsection{Reciprocal space}

We have seen, in the previous section, how to describe atomic positions in crystals and the role of the symmetries in this task. If one now wants to determine experimentally a crystallographic structure, diffraction measurements can be carried out. Intuitively, such experiments are based on constructive interference of waves that for a given wavevector of incident beam of X-rays or neutrons amplifies scattering in directions for which conditions of equiphase with specific periods are encountered. This naturally leads to a dual picture of the crystal structure, where, typically but not only, a line (a plane) in the real space for instance gets associated to a perpendicular plane (a line) in the dual space. As a matter of fact, the scattering amplitudes are computed as coefficients of the Fourier transform of the atomic charge or nuclear densities (and of magnetic densities if any). It is customary to call the dual space reciprocal space. This Section is devoted at defining the reciprocal space (some examples will be given), giving its most important properties. It ends with a brief introduction to the concept of Brillouin zone.

\subsubsection{Definitions and examples}

The reciprocal lattice is defined as a network of points in the Fourier space (dual) which are the extremities of vectors:

$$
\vec{\tau}=h \vec{a}^{\star}+k \vec{b}^{\star}+l \vec{c}^{\star}
$$


- a -

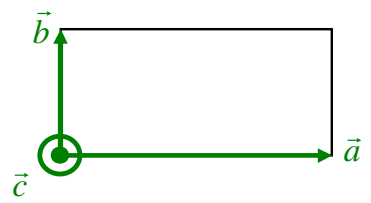

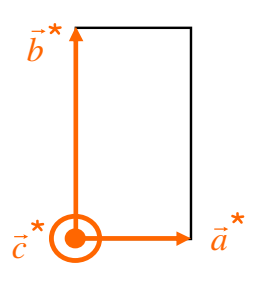

$-b-$

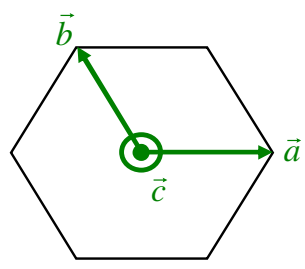

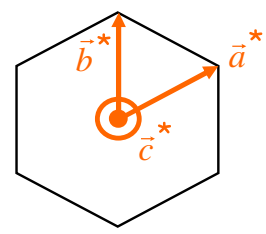

Figure 18. (Color online) Examples of direct cell (left figure, in green) and reciprocal cell (right figure, in orange) for two different conventional cells: a- orthorhombic and b- hexagonal.

with $h, k, l$ integers and $\vec{a}^{\star}, \vec{b}^{\star}, \vec{c}^{\star}$ the basis vectors of the reciprocal lattice. The latter are defined as follows:

$$
\vec{a}^{\star}=C \frac{\vec{b} \wedge \vec{c}}{V}, \quad \vec{b}^{\star}=C \frac{\vec{c} \wedge \vec{a}}{V}, \quad \vec{c}^{\star}=C \frac{\vec{a} \wedge \vec{b}}{V}
$$

with $C$ a constant and $V$ the volume of the unit cell in direct space defined by:

$$
\begin{aligned}
& C= \begin{cases}2 \pi & \text { in solid states physics } \\
1 & \text { in crystallography }\end{cases} \\
& V=(\vec{a}, \vec{b}, \vec{c})=(\vec{a} \wedge \vec{b}) \cdot \vec{c}=\vec{a} \cdot(\vec{b} \wedge \vec{c})
\end{aligned}
$$

From definitions (2.26), one immediately sees that $\vec{a}^{\star}$ is perpendicular to $\vec{b}$ and $\vec{c}, \vec{b}^{\star}$ to $\vec{c}$ and $\vec{a}$, and $\vec{c}^{\star}$ to $\vec{a}$ and $\vec{b}$. More precisely, using the convention of crystallographers $(C=1)$, eq. (2.26) yield the following properties: ${ }^{7}$

$$
\begin{aligned}
& \vec{a}^{\star} \cdot \vec{a}=\vec{b}^{\star} \cdot \vec{b}=\vec{c}^{\star} \cdot \vec{c}=1 \\
& \vec{a}^{\star} \cdot \vec{b}=\vec{a}^{\star} \cdot \vec{c}=0 \\
& \vec{b}^{\star} \cdot \vec{a}=\vec{b}^{\star} \cdot \vec{c}=0 \\
& \vec{c}^{\star} \cdot \vec{a}=\vec{c}^{\star} \cdot \vec{b}=0
\end{aligned}
$$

As an illustration, figure 18 shows the direct and reciprocal cell for two different conventional unit cells: orthorhombic (a) and hexagonal (b). For the orthorhombic unit cell (of direct volume $V=a b c$ ), the reciprocal space is simply such as: $\vec{a}^{\star} \| \vec{a}$ with $a^{\star}=1 / a, \vec{b}^{\star} \| \vec{b}$ with $b^{\star}=1 / b$, and $\vec{c}^{\star} \| \vec{c}$ with $c^{\star}=1 / c$, and is thus also orthorhombic. For the hexagonal unit cell (of direct volume $V=\sqrt{3} a^{2} c / 2$ ), $\vec{a}^{\star}$ and $\vec{b}^{\star}$ are not parallel to $\vec{a}$ and $\vec{b}$, respectively (see Fig. 18-b), and $a^{\star}=b^{\star}=2 /(a \sqrt{2}$ ), while $\vec{c}^{\star} \| \vec{c}$ ( since $\vec{c} \perp \vec{a}, \vec{b}$ ) and $c^{\star}=1 / c$. The reciprocal cell is thus also hexagonal but with $\gamma^{\star}=60^{\circ}$ (to be compared to $\gamma=120^{\circ}$ in direct space). More generally, the reciprocal cell of the direct cell always belongs to the same conventional cell.

\subsubsection{Properties}

The properties of the direct space and the definition of the reciprocal space yield particular properties. A few of them (the most crucial) will be recalled here.

- The reciprocal lattice of the reciprocal lattice is the direct space lattice.

- The direct space is used to describe the atomic positions in the crystal, the reciprocal space is used to describe the positions of the diffracted peaks, phonons, magnons, $\cdots$

\footnotetext{
${ }^{7}$ Eq. (2.29) can be taken as the defining relations for a dual lattice. They easily are solved to give eq. (2.26).
} 


\section{Contribution of Symmetries in Condensed Matter}

- Each family of rows $[h k l]^{\star}$ in the reciprocal space is perpendicular to the family of net planes $(h k l)$ in the direct space, and each family of net planes $(u v w)^{\star}$ in the reciprocal space is perpendicular to the family of rows $[u v w]$ in the direct space.

- Using the definition of the reciprocal space, the $d$-spacing for a family $(h k l)$ of net planes in the direct space is simply:

$$
d_{h k l}=\frac{1}{|\vec{Q}|}=\frac{1}{\sqrt{\left(h \vec{a}^{\star}+k \vec{b}^{\star}+l \vec{c}^{\star}\right) \cdot\left(h \vec{a}^{\star}+k \vec{b}^{\star}+l \vec{c}^{\star}\right)}}
$$

with $\vec{Q}$ the scattering vector, defined in diffraction as $\vec{Q}=\vec{k}_{f}-\vec{k}_{i}$, where $\vec{k}_{i}$ and $\vec{k}_{f}$ are respectively the initial and final wave vectors of the X-ray or neutron beam.

- The effect in the reciprocal space of a symmetry operation in the direct space is the following: in a standard diffraction experiment, $\mathrm{i}$ - the symmetry of the reciprocal space is given by the Laue class (i.e. the inversion is always present) while ii- the extinction rules result from the glide translations contained in non-symmorphic space group symmetries. Let us illustrate this by two examples:

Example 1. Let us consider a space group containing an axis $2_{1} \| \vec{b}$ (the Laue class thus contains an axis $2 \| \vec{b}$ ). i- This yields an axis $2 \| \vec{b}^{\star}$ in reciprocal space (effect of the Laue class), so that Bragg peaks $h k l$ and $\bar{h} k \bar{l}$ will have the same intensity. Note that due the centrosymmetry of the reciprocal space, Bragg peaks $\bar{h} \bar{k} \bar{l}$ and $h \bar{k} l$ will also have the same intensity as the former two. ii- The glide translation of the $2_{1}$ axis, $\vec{t}=\frac{1}{2} \vec{b}$, yields a translation $\vec{t}^{\star}=2 \vec{b}^{\star}$ along the equivalent axis passing though the origin in the reciprocal space (effect of a non symmorphic space group symmetry), so that $0 k 0$ Bragg peaks with $k$ odd will be forbidden.

Example 2. Let us now consider a space group containing a glide plane $a \perp \vec{b}$ (the Laue class thus contains a mirror $m \perp \vec{b}$ ). i- This yields a mirror $m \perp \vec{b}^{\star}$ in reciprocal space, so that Bragg peaks $h k l$ and $h \bar{k} l$ will have the same intensity, as well as $\bar{h} \bar{k} \bar{l}$ and $\bar{h} k \bar{l}$ due to the presence of an inversion center in the reciprocal space. ii- The glide translation of the $a$ plane, $\vec{t}=\frac{1}{2} \vec{a}$, yields a translation $\vec{t}^{\star}=2 \vec{a}^{\star}$ parallel to the equivalent plane passing through the origin in the reciprocal space, so that $h 0 l$ Bragg peaks with $h$ odd will be forbidden.

The extinction rules (or reflection conditions) can be found in the International Tables for Crystallography [1], as can be seen in the right column on Figures 15 (see (14)) and 17. The general reflection conditions are first given in front of the general Wyckoff site: they arise from the presence in the considered space group of non symmorphic symmetry operations and thus concern all atoms. If for a Bragg reflection, one of these reflection conditions is not fulfilled, then the diffracted intensity is null. Then the special conditions are given, in front of each special Wyckoff site: for some sites, no additional reflection condition is mandatory, while for some others, an additional reflection condition is given. The contribution to the diffracted intensity of the atoms occupying a specific Wyckoff position is null if the additional special reflection condition listed here is not fulfilled. Let us focus on the example of Pnma (see (14) in Fig. 15). The first general condition ' $0 k l: k+l=2 n$ ' comes from the $n$ plane perpendicular to $\vec{a}$ and is to be read as follows: a necessary condition for a Bragg peak $0 \mathrm{kl}$ not to be null is that $k+l$ must be even. But this does not mean that all $0 k l$ peaks with $k+l=2 n$ will have a non zero intensity (the structure factor could, for a particular motif and particular $k$ and $l$ values, give zero intensity). ' $h k 0: h=2 n$ ' comes from the presence of a $a$ plane perpendicular to $\vec{c}$, while ' $h 00: h=2 n$ ', ' $0 k 0: k=2 n$ ', and ' $00 l: l=2 n$ ' come from the presence of $2{ }_{1}$ axes along $\vec{a}, \vec{b}$, and $\vec{c}$, respectively.

Let us now briefly comment the reflection conditions listed for the space group I4mm (see Fig. 17). This space group is non symmorphic. Nevertheless, it has some extinction rules. The latter arise from the choice of a body-centered unit cell: $h+k+l$ even is a necessary condition for a Bragg peak $h k l$ not to be null (first reflection condition in Fig. 17). The other extinctions rules, displayed below, are just consequences of the first one. More generally, a non primitive lattice (multiplicity $m=2,3$, 
or 4), contrary to a primitive one, always shows an extinction rule on $h k l$ Bragg peaks. This 'artificial' extinction rule simply comes from the fact that a cell $m$ times too large is chosen in direct space, so that the reciprocal cell is $m$ times too small. This means that $m-1$ reciprocal lattice points out of $m$ do actually not exist so should result in zero intensity for any elastic scattering.

\subsubsection{The Brillouin zone}

It at last is worth recalling the concept of Brillouin zone, which is very useful when dealing with diffraction and propagating phenomena (phonons, magnons, ...) or electronic structure of matter. One defines the first Brillouin zone as the smallest polyhedron enclosed by the perpendicular bisectors of the nearest neighbors to a given point of the reciprocal space. The first Brillouin zone thus is the equivalent in the reciprocal space of the Wigner-Seitz cell in the direct space. By construction, $i-$ the whole reciprocal space is filled by locating a first Brillouin zone at each reciprocal lattice point, ii- the first Brillouin zone has the same volume as the primitive cell in the reciprocal space. Examples of first Brillouin zones are displayed figure 19. A first Brillouin zone often contains points of higher symmetry, called special points. These are marked with a particular symbol (see Fig. 19-c). One for instance distinguishes the points

$\begin{array}{ll}\text { Г: } & \text { Brillouin zone's center } \\ \text { K: } & \text { middle of an edge between two hexagonal faces } \\ \text { L: } & \text { center of an hexagonal face } \\ \mathrm{U}: & \text { middle of an edge between a square face and an hexagonal one } \\ \mathrm{X}: & \text { center of a square face } \\ \mathrm{W}: & \text { corner }\end{array}$
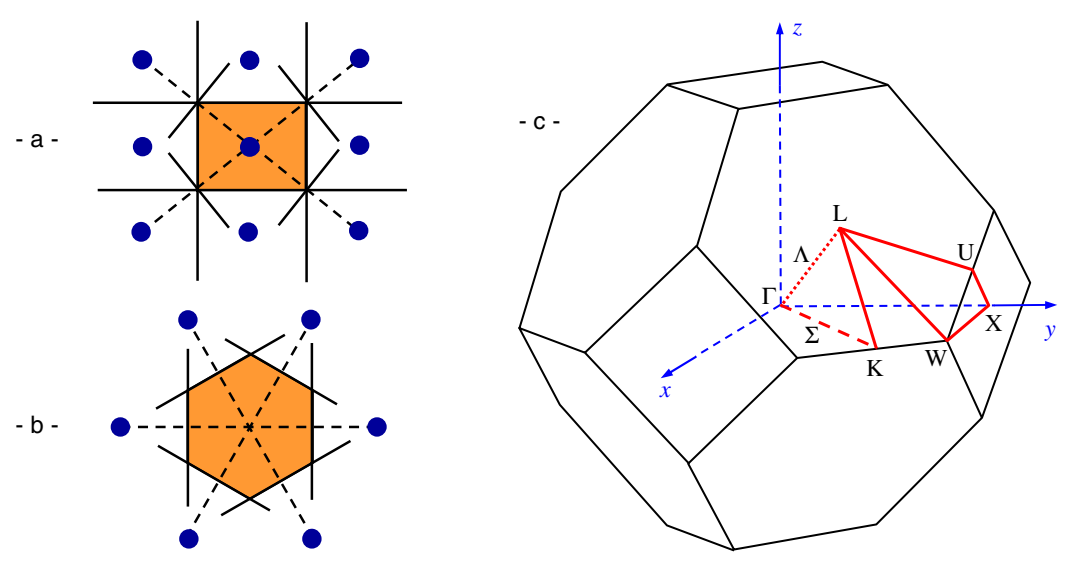

Figure 19. First Brillouin zone for three different reciprocal lattices: a- two-dimensional rectangular, b- twodimensional hexagonal, c- three dimensional cubic $F$. In figures a and b, the reciprocal lattice points are the solid blue circles and the first Brillouin zone is colored in orange with the bisectors in black solid lines. In figure c, the first Brillouin zone is delimited by the black solid lines, $x, y, z$ indicate the directions of $\vec{a}^{\star}, \vec{b}^{\star}, \vec{c}^{\star}$, and special positions are labeled by capital letters corresponding to a standard notation (see text).

One more generally defines an $n^{\text {th }}$ Brillouin zone in the reciprocal space as the set of points that can be reached from the origin by crossing exactly $n-1$ Bragg planes. A Bragg plane is a plane that bisects a reciprocal lattice vector. All the Brillouin zones have the same volume. The relevance of the Brillouin 


\section{Contribution of Symmetries in Condensed Matter}

zones for wave propagation in a crystal is to bring to light all the incident wavevectors $\vec{k}_{i}$ to which the crystal can make undergo a diffraction to $\vec{k}_{i}+\vec{\tau}$, where $\vec{\tau}$ is the reciprocal lattice vector. The condition for Bragg reflection $\vec{k}_{f}-\vec{k}_{i}=\vec{\tau}$ indeed may write $\vec{k}_{i} \cdot \frac{1}{2} \vec{\tau}=\left(\frac{1}{2} \vec{\tau}\right)^{2}$, using the fact that diffraction is elastic and the Friedel law.

\section{REPRESENTATION ANALYSIS}

A crystal is not solely a geometric entity specified by a space group symmetry. It is made of atoms, ions or molecules that mutually interact in a number of ways or react to surrounding fields, local and external. It accordingly exhibits a variety of static and dynamical physical properties that find transcriptions into a wide range of physical quantities, namely thermodynamic variables featuring a macrostate, wavefunctions or fields describing quantal or classical microscopic states or excitations, response functions to experimental probes, .... A macroscopic strain, an electric polarization, ..., a wavefunction of an individual atom, ion or molecule in its crystal environment, an exciton wavefunction, ..., a magnetic moment configuration field, an electric quadrupole tensor field, ..., a magneto-electric susceptibility tensor, a dynamical neutron scattering function, ... are a few among typical examples. It is explicit from this enumeration that a physical quantity may be of diverse nature, tensorial or spinorial possibly function of time and space, so prone to change under time or space transforms. When these belong to a symmetry group it is of crucial interest to establish under which conditions a particular quantity might be invariant, or to specify the orbit of all its transforms in the instance where it inherently would be of lower symmetry, ${ }^{8}$ or else to gauge the fully or partially symmetric components out of which it is compounded. A natural and elegant mathematical framework to efficiently achieve these tasks is group action or, equivalently, group representation. Unfortunately, explaining the associated formalism in details and with rigor requires to define a series of abstract concepts, demonstrate a number of theorems and describe a variety of unfamiliar algorithmic constructions that often makes it abstruse, thus hiding the power of the method. Attempt will be made in the following to provide an intuitive insight. More detailed and technical descriptions may be consulted in the refs. [7-11]. Section 3.1 discusses at the most elementary level the few concepts necessary to understand or to perform analysis of finite group linear representations with the help of already tabulated or computer generated irreducible representations [6, 8-13]. The method is applied to molecule vibrations. Section 3.2 provides a qualitative description of the irreducible representations of the 3-dimensional crystal symmetry groups. No time transform is considered. Notice that if the physical property is dynamical but is not slaved to an external explicitly time dependent field then it ought to be stationary if it is at thermodynamic equilibrium, in which case it does not depend on an initial time, in particular dynamical correlations then involve only time intervals. When time reversal is possibly relevant, it is dealt with in the context of co-representations of groups [14] (see also chapter by Schweizer).

\footnotetext{
8 According to the Curie's principle a physical effect cannot have an asymmetry absent from its efficient cause. So one would have expected only full invariance of the physical quantities which otherwise would be excluded from materialization, but this would lead to a too poor universe. As a matter of fact, "c'est la dissymétrie qui crée le phénomène ". A symmetry breaking might be merely externally imposed, for instance by applying a stress, an electric field, a magnetic field, ..., but, most interestingly, it may occur spontaneously, for instance in bifurcation phenomena or in phase transitions, ..., owing to space-time correlations in the physical properties albeit the interactions producing these are themselves symmetric. New phases and associated new physical quantities (order parameters) emerge in this case. All of the forms of these, as transformed into one another by the broken symmetries, are equiprobable and therefore should coexist, which gives rise to domains and symmetry restoring dynamic modes called Nambu-Goldstone. The Curie's principle thus may be extended to include the spontaneous symmetry breaking phenomena. It would state that a cause might produce effects of lower symmetry provided that when these all coexist then the whole become as symmetric as the cause.
} 


\subsection{Qualitative approach}

An analysis of representation, in practice, consists at first to identify which symmetry group $\mathrm{G}$ is under concern. This generally is obvious when the symmetries are geometric. If we are interested in the effects of the orientation invariance of a molecule or a crystal then its point group imposes itself. If the discrete translation invariance of a crystal is involved, as with any spatial modulation of atomic or molecule states or any phenomenon propagating within the crystal, then the space group must be considered. It is emphasized that symmetries other than purely geometric might exist, emerging for instance through invariance of dynamics, which are less evident to discern and are here merely ignored.

The next stage is to determine the relevant representation space $\mathrm{V}$ of the investigated quantity. This is a vector space each element of which describes a distinct value or functional form of the quantity. It does not always reveal itself obviously and should be defined carefully to provide the correct intuitions. This essentially is where physics stands, all the rest being mathematics. It often proves useful to build $\mathrm{V}$ from direct sums or tensor products of more elementary vector spaces. The representation space of the magnetic configurations in a magnetic crystal for instance may be build as the direct sum $\mathrm{V}=\bigoplus_{\text {in }} \mathrm{V}_{\text {in }}$ of the representation spaces $\mathrm{V}_{\text {in }}$ of the magnetic moment $\vec{s}_{i n}$ on the magnetic position (in) ( $i$ : motif index, $n$ : lattice index). Every magnetic configuration in the crystal then is fully determined by the vector $\vec{v}=\bigoplus_{i n} \vec{s}_{i n}$ in V. Addition of configurations and multiplications by a real or a complex scalar then are evident as well the vector space structure of $\mathrm{V}$. Note that the dimension of $\mathrm{V}$ is immediately obtained as $d=\operatorname{dim}(\mathrm{V})=\sum_{i n} d_{i n}$, where $d_{i n}$ is the dimension of each $\mathrm{V}_{i n}$.

It matters then to transcribe the symmetries of the group $\mathrm{G}$ in the representation space $\mathrm{V}$. If a space transformation $g \in \mathrm{G}$ is performed then each element of $\mathrm{V}$, as a tensor quantity or a function of space, is transformed into another in $\mathrm{V}$, which defines a necessarily one-to-one $g$-dependent transformation $\rho(g): \mathrm{V} \rightarrow \mathrm{V}$. As a matter of fact, in all the cases that we might encounter, $\rho(g)$ is an invertible linear operator on $\mathrm{V}$, that is to say

$$
\rho(g)(a \vec{u}+b \vec{v})=a \rho(g)(\vec{u})+b \rho(g)(\vec{v}) \quad \forall a, b \in \mathbb{C} \quad \forall \vec{u}, \vec{v} \in \mathrm{V}
$$

It also is generally found out that these linear operators obey to the homomorphism rule:

$$
\rho(g h)=\rho(g) \circ \rho(h) \quad \forall g, h \in \mathrm{G}
$$

It is in that sense that the application $\rho: g \mapsto \rho(g)$ is said to form a linear representation of the group $\mathrm{G}$ on the representation space V. Let $\left\{\hat{e}_{m}\right\}_{m=1, \cdots, d}$ stand for a basis in V. One may write

$$
\rho(g)\left(\hat{e}_{m}\right)=\sum_{n} \hat{e}_{n} \Gamma(g)_{n m}
$$

where the $d \times d$ invertible matrix $\Gamma(g)$ is called the matrix representative of the linear operator $\rho(g)$. Note that if $\vec{v}=\sum_{m} x_{m} \hat{e}_{m}$ and $\rho(g)(\vec{v})=\sum_{n} y_{n} \hat{e}_{n}$ then $y_{n}=\sum_{m} \Gamma(g)_{n m} x_{m}$. Eq. (3.2) implies that

$$
\Gamma(g h)=\Gamma(g) \Gamma(h) \quad \forall g, h \in \mathrm{G}
$$

The application $\Gamma: g \mapsto \Gamma(g)$ of the group $\mathrm{G}$ to the group $\operatorname{GL}(d, \mathbb{C})$ of $d \times d$ invertible matrices makes up a matrix materialization of the linear representation $\rho$ of the group G. It defines a matrix representation of the group $\mathrm{G}$. The choice of another basis $\left\{\hat{f}_{m}\right\}$ would have led to another matrix representation $\Lambda: g \mapsto \Lambda(g)$ such that

$$
\Lambda(g)=S \Gamma(g) S^{-1} \quad \forall g \in \mathrm{G}
$$

where $S$ is the invertible matrix associated with the basis change $\left\{\hat{e}_{m}\right\} \rightarrow\left\{\hat{f}_{m}\right\}$. This defines a similarity transformation and the two matrix representations are said equivalent: $\Gamma \sim \Delta$. A quantity exists that fully determines the matrix representation up to this equivalence. It is called the character $\chi$ of the 


\section{Contribution of Symmetries in Condensed Matter}

matrix representation $\Gamma$ and is the function on the group $\mathrm{G}$ defined as

$$
\chi(g)=\operatorname{Tr}[\Gamma(g)]=\sum_{i} \Gamma_{i i}(g) \quad \forall g \in \mathrm{G}
$$

It is the invariance of the trace Tr over cyclic permutation of operators that makes the character invariant with respect to every basis change. This property implies also that the character is constant over the conjugacy classes of G. It is recalled that two elements $g$ and $h$ of a group $\mathrm{G}$ belong to the same conjugacy class if and only if there exists another element $t$ in the group G such that $h=\operatorname{tgt}^{-1}$.

A quantitative value or a functional form of the physical quantity under concern is explicitly specified by picking out a vector $\vec{v}$ from the representation space V. Its transforms under the symmetries of a group $\mathrm{G}$ are obtained by building up its orbit $\operatorname{Orb}(\vec{v})=\{\rho(g)(\vec{v}) \mid g \in \mathrm{G}\}$, namely the set of its images by the linear operators $\rho(g)$ for $g$ running over the group $\mathrm{G}$. $\operatorname{Orb}(\vec{v})$ spans a vector space $\mathrm{U}$, by the linear combinations of its elements every which way. Most choices of $\vec{v}$ lead to $\mathrm{U}=\mathrm{V}$. It however may happen that $\mathrm{U}$ is of dimension $d_{\mathrm{U}}$ strictly lower than the dimension $d$ of $\mathrm{V}$ but not null: $0<d_{\mathrm{U}}<d$. $\mathrm{U}$ then is an invariant subspace by the group $\mathrm{G}$, that is to say $\forall g \in \mathrm{G}$ if $\vec{u} \in \mathrm{U}$ then $\rho(g)(\vec{u}) \in \mathrm{U}{ }^{9}$ The restriction $\rho_{\mathrm{U}}$ of $\rho$ to the subspace $\mathrm{U}$ makes up a linear representation of the group G. According to the Maschke's Theorem, in the usual cases we might encounter, there always exist a complement $\mathrm{W}$ of $\mathrm{U}$ in the representation space $\mathrm{V}$, that is such that $\mathrm{V}=\mathrm{U} \oplus \mathrm{W}$, which is also invariant by $\mathrm{G}$. The restriction $\rho_{\mathrm{W}}$ of $\rho$ to the subspace $\mathrm{W}$ makes up another linear representation. Since $\mathrm{V}=\mathrm{U} \oplus \mathrm{W}$, a basis is built in $\mathrm{V}$ from the union of a basis of $\mathrm{U}$ and a basis of $\mathrm{W}$ and since $\mathrm{U}$ and $\mathrm{W}$ are separately invariant by $\mathrm{G}$ the matrix representatives $\Gamma(g)$ of the linear operators $\rho(g)$ with respect to this basis writes in the same block diagonal form for every $g \in \mathrm{G}$, more precisely as the direct sum $\Gamma^{\mathrm{U}}(g) \oplus \Gamma^{\mathrm{W}}(g)$ where $\Gamma^{\mathrm{U}}(g)\left(\right.$ resp. $\left.\Gamma^{\mathrm{W}}(g)\right)$ is the matrix representative of the linear operator $\rho_{\mathrm{U}}(g)$ (resp. $\rho_{\mathrm{W}}(g)$ ) with respect to the basis selected in $\mathrm{U}$ (resp. in $\mathrm{W}$ ). Generalization to the cases where the already identified invariant subspace or its complement contains in turn invariant subspaces is straightforward. It suffices to consider the restrictions of the linear representation to each of the found out invariant subspaces and select basis vectors separately in each of these to get the matrix representatives in block diagonal forms:

$$
\Gamma(g)=\Gamma^{1}(g) \oplus \Gamma^{2}(g) \oplus \cdots \oplus \Gamma^{n}(g) \equiv\left(\begin{array}{cccc}
\Gamma^{1}(g) & 0 & \cdots & 0 \\
0 & \Gamma^{2}(g) & \cdots & 0 \\
\vdots & \vdots & \ddots & \vdots \\
0 & 0 & \cdots & \Gamma^{n}(g)
\end{array}\right) \quad \forall g \in \mathrm{G}
$$

One says that the matrix representation $\Gamma$ is the direct sum $\Gamma^{1} \oplus \Gamma^{2} \oplus \cdots \Gamma^{n}$ of $n$ matrix (sub-) representations $\Gamma^{i}$.

The process of block diagonalization stops when no further invariant subspace can be found out. A matrix representation $\Gamma^{i}$ that cannot be decomposed into a block diagonal form is called an irreducible matrix representation. The linear representation $\rho_{i}$ to which it is associated by a basis choice is also said irreducible. Note that several among the $\Gamma^{i}$ might be equivalent with each other. The most general decomposition of a matrix representation $\Gamma$ into irreducible matrix representations thus may be written symbolically as

$$
\Gamma \sim n_{1} \Gamma^{1} \oplus n_{2} \Gamma^{2} \oplus \cdots n_{s} \Gamma^{s}=\bigoplus_{k} n_{k} \Gamma^{k}
$$

\footnotetext{
${ }^{9}$ Consider for instance a charge distribution described in the spherical coordinates $(r, \theta, \varphi)$ by functional forms $\mathcal{F}(r) \mathcal{G}(\theta, \varphi)$. These belong to a function space of dimension $d=\infty(d$ needs not be finite !). Under the spatial rotations $\mathcal{R}(\hat{u}, \Theta)$ the angular part $\mathcal{G}(\theta, \varphi)$ gets transformed into $\mathcal{G}(\mathcal{R}(\hat{u},-\Theta)(\theta, \varphi))$, which gives rise to an orbit of functional forms. It is only when the charge distribution is special that the function space generated from this orbit is finite dimensional. A distribution described by a spherical harmonic of order $l, \mathcal{F}(r) \mathcal{G}(\theta, \varphi) \equiv \mathcal{F}(r) Y_{l}^{m}(\theta, \varphi)$, satisfies this condition, in which case the invariant subspace is of dimension $2 l+1$.
} 
where $n_{k}$ is the multiplicity of the irreducible component $\Gamma^{k}$ contained in $\Gamma$. The ultimate aim of representation analysis is to obtain this decomposition. We shall admit without demonstration the Orthogonality Theorem for the characters $\chi^{k}$ of the irreducible representations $\Gamma^{k}$ :

$$
\frac{1}{n_{\mathrm{G}}} \sum_{g \in \mathrm{G}}\left(\chi^{q}(g)\right)^{\star} \chi^{k}(g)=\left\langle\chi^{q} \mid \chi^{k}\right\rangle=\delta_{k q}
$$

The multiplicities $n_{k}$ then are deduced as

$$
n_{k}=\frac{1}{n_{\mathrm{G}}} \sum_{g \in \mathrm{G}} \chi(g)^{\star} \chi^{k}(g)=\left\langle\chi \mid \chi^{k}\right\rangle
$$

Note that $\langle\chi \mid \chi\rangle=\sum_{k} n_{k}^{2}$. We conclude that a matrix representation $\Gamma$ is irreducible if and only if the norm of its character $\chi$ equals $\langle\chi \mid \chi\rangle=1$. This provides an extremely practical criterion of irreducibility. Once the decomposition displayed in eq. (3.8) is obtained, it matters to determine the invariant subspaces $\mathrm{V}_{\kappa=1, \cdots, n_{k}}^{k}$ associated with each occurrence $\kappa$ of the irreducible representation $\Gamma^{k}$ in $\Gamma$. It is shown that the projection $(m=n)$ and shift $(m \neq n)$ operators

$$
p_{n m}^{k}=\frac{d_{k}}{n_{\mathrm{G}}} \sum_{g \in \mathrm{G}} \Gamma_{n m}^{k}\left(g^{-1}\right) \rho(g)
$$

allow performing the task: basis vectors $\hat{e}_{n \kappa}^{k}$ of $\mathrm{V}_{\kappa}^{k}$ can be obtained by applying the projectors $p_{n n}^{k}$ to trial vectors $\hat{e}$ of $\mathrm{V}$ whereas by applying the exchangers $p_{n m \neq n}^{k}$ to any given basis vector $\hat{e}_{n \kappa}^{k}$ of $\mathrm{V}_{\kappa}^{k}$ another basis vector $\hat{e}_{m \kappa}^{k}$ of $\mathrm{V}_{\kappa}^{k}$ can be deduced. If the irreducible matrix representation $\Gamma^{k}$ is contained more than once $\left(n_{k}>1\right)$ in the matrix representation $\Gamma$ then the projectors $p_{n n}^{k}$ must be applied to as many trial vectors $\hat{e}$ of $\mathrm{V}$ as necessary to generate $n_{k}$ basis vectors that cannot be transformed into each other by the shift operators $p_{n m \neq n}^{k}$.

\subsubsection{An elementary example}

Let us illustrate what precedes by the simple example of molecule vibrations. We consider the ammonia molecule $\mathrm{NH}_{3}$ described in subsection 2.1.3. Its symmetry group is $\mathrm{G}=3 \mathrm{~m}$. We are interested in the motion of the $N$ atoms ( $N=4$ for the $\mathrm{NH}_{3}$ molecule). If $\mathbb{R}^{3}$ stands for the ordinary space then a natural choice for the representation space may be $\mathrm{V}=\bigoplus_{i=1, \cdots, N} \mathbb{R}^{3} \equiv \mathbb{R}^{3 N}$. We are free to define in $\mathrm{V}$ the basis $\left\{\hat{e}_{i \alpha}\right\}$ such that if $\vec{v}=\sum_{i \alpha} v_{i \alpha} \hat{e}_{i \alpha}$ then $\hat{e}_{i \alpha} \cdot \vec{v}=v_{i \alpha}$ describes the motion of the $i$ atom along the $\alpha$ direction $(i=1, \ldots, N$ and $\alpha=x, y, z)$. Under every geometric transformation $g$ of $\mathrm{G}$ the atoms are exchanged and the displacements $v_{i \alpha}$ are rotated or roto-inverted. It is not difficult to get convinced that this defines invertible linear operators $\rho(g)$ on the vector space V obeying the homomorphism rule $\rho(g h)=\rho(g) \circ \rho(h) \forall g, h \in \mathrm{G}$, that is to say a linear representation $\rho$ of $\mathrm{G}$ on $\mathrm{V}$. One more concretely has

$$
\rho(g) \vec{v}=\sum_{i \alpha \beta} \Gamma_{\mathrm{v}}(g)_{\alpha \beta} v_{\left(g^{-1} i\right) \beta} \hat{e}_{i \alpha}=\sum_{i j \alpha \beta} \Gamma_{\mathrm{v}}(g)_{\alpha \beta} \Gamma_{\mathrm{p}}(g)_{i j} v_{j \beta} \hat{e}_{i \alpha}=\sum_{i j \alpha \beta} \Gamma(g)_{i j \alpha \beta} v_{j \beta} \hat{e}_{i \alpha}
$$

$v_{\left(g^{-1} i\right) \beta}$ stands for the component along $\beta$ of the displacement of the atom $j=g^{-1} i$ that by application of $g$ gets into the position of the atom $i$ and is rotated or roto-inverted accordingly. $\Gamma_{\mathrm{v}}(g)$ is the matrix representative of a rotation or a rotoinversion in the real space $\mathbb{R}^{3}$. As an example for a rotation $\mathcal{R}(\hat{z}, \varphi)$ of angle $\varphi$ about the $\hat{z}$-axis, one would have

$$
\Gamma_{\mathrm{v}}(\mathcal{R}(\hat{z}, \varphi))=\left(\begin{array}{ccc}
\cos \varphi & -\sin \varphi & 0 \\
\sin \varphi & \cos \varphi & 0 \\
0 & 0 & 1
\end{array}\right)
$$




\section{Contribution of Symmetries in Condensed Matter}

with respect to the basis $\{\hat{x}, \hat{y}, \hat{z}\}$. If $\chi_{\mathrm{v}}$ denotes the character of $\Gamma_{\mathrm{v}}$ then $\chi_{\mathrm{v}}(\mathcal{R}(\hat{z}, \varphi))=$ $\operatorname{Tr}\left[\Gamma_{\mathrm{v}}(\mathcal{R}(\hat{z}, \varphi))\right]=(1+2 \cos \varphi)$. Composing this rotation with the inversion $\mathcal{I}$ we get the rotoinversion $\mathcal{I} \circ \mathcal{R}(\hat{z}, \varphi)$ to which the matrix representative $\Gamma_{\mathrm{v}}(\mathcal{I} \circ \mathcal{R}(\hat{z}, \varphi))=-\Gamma_{\mathrm{v}}(\mathcal{R}(\hat{z}, \varphi))$ with respect to the same basis is associated, so that $\chi_{\mathrm{v}}(\mathcal{I} \circ \mathcal{R}(\hat{z}, \varphi))=\operatorname{Tr}\left[\Gamma_{\mathrm{v}}(\mathcal{I} \circ \mathcal{R}(\hat{z}, \varphi))\right]=-(1+2 \cos \varphi)$. Now, it is recalled that the trace of a matrix is invariant by any basis change. It follows that $\chi_{\mathrm{v}}(\mathcal{R}(\hat{u}, \varphi))=(1+2 \cos \varphi)$ and $\chi_{\mathrm{v}}(\mathcal{I} \circ \mathcal{R}(\hat{u}, \varphi))=-(1+2 \cos \varphi)$ whatever the orientation of the rotation $\hat{u}$-axis, because we can always find a basis with $\hat{u}$ as a $\hat{z}$-axis. $\Gamma_{\mathrm{p}}(g)$ is the matrix representative of the $g^{-1} i \mapsto i$ transform. Its matrix elements are given as

$$
\Gamma_{\mathrm{p}}(g)_{i j}=\delta\left(g^{-1} i, j\right)
$$

where $\delta$ is the Kronecker symbol: $\delta(a, b)=1$ if and only if $a=b$ and 0 otherwise. The character $\chi$ of the matrix representation $\Gamma$ then is easily deduced as being given by

$$
\chi(g)=\operatorname{Tr}[\Gamma(g)]=\operatorname{Tr}\left[\Gamma_{\mathrm{v}}(g) \otimes \Gamma_{\mathrm{p}}(g)\right]=\chi_{\mathrm{v}}(g) \chi_{\mathrm{p}}(g)= \pm \mathcal{N}(g)(1+2 \cos \varphi)
$$

with the + sign for a rotation and the - sign for a rotoinversion, and where $\otimes$ symbolizes matrix tensor product. $\varphi$ is the rotation angle associated with $g$ and $\mathcal{N}(g)$ the number of atoms invariant under $g$.

The variables $v_{j \beta}$ account for not only vibrations, but also for the translation $(\mathrm{T})$ and rotation $(\mathrm{R})$ of the molecule as a whole. These global motions determine the total linear $\vec{P}$ and angular $\vec{L}$ momenta of the molecule and belong to subspaces $\mathrm{V}_{\mathrm{T}}$ and $\mathrm{V}_{\mathrm{R}}$ of $\mathrm{V}$ that are stable under $\mathrm{G}$, because vibrating motions never contribute to $\vec{P}$ nor to $\vec{L}$ by conservation of these quantities. It follows that

$$
\Gamma(g)=\Gamma_{\mathrm{T}}(g) \oplus \Gamma_{\mathrm{R}}(g) \oplus \Gamma_{\mathrm{vib}}(g)
$$

$\Gamma_{\mathrm{T}}(g)$ operates on polar vectors $\overrightarrow{\mathrm{T}}$ of global translation and $\Gamma_{\mathrm{R}}(g)$ on axial vectors $\vec{\Omega}$ of global rotation $\left(\vec{\Omega} \wedge \sum_{i} \overrightarrow{\mathrm{OM}}_{i}\right.$ defines the displacement of the atoms, initially at the positions $M_{i}$, associated with the rotation of angle $\Omega$ about an axis along $\vec{\Omega}$ crossing the center of gravity $\mathrm{O}$ of the molecule). Thus if $\chi_{\mathrm{T}}$ is the character of $\Gamma_{\mathrm{T}}$ and $\chi_{\mathrm{R}}$ the character of $\Gamma_{\mathrm{R}}$ then $\chi_{\mathrm{T}}(g)= \pm(1+2 \cos \varphi)$ and $\chi_{\mathrm{R}}(g)=(1+2 \cos \varphi)$. As a result the character, $\chi_{\text {vib }}$ of $\Gamma_{\text {vib }}$ is given as

$$
\chi_{\text {vib }}(g)= \begin{cases}(\mathcal{N}(g)-2)(1+2 \cos \varphi) & \text { if } g \text { is a pure rotation } \\ -\mathcal{N}(g)(1+2 \cos \varphi) & \text { if } g \text { is a rotoinversion }\end{cases}
$$

Now let us consider back the group $\mathrm{G}=3 \mathrm{~m}$ of the ammonia molecule. It contains 6 elements distributed over 3 classes: $\{e\}, \mathrm{C}_{1}=\left\{3,3^{2}\right\}, \mathrm{C}_{2}=\left\{m, m 3, m 3^{2}\right\}$. It then owns 3 irreducible representations $\Gamma^{0}, \Gamma^{1}$ and $\Gamma^{2}$. One indeed may show that for any finite group the number of its irreducible representations is equal to the number of its conjugacy classes. One also establishes that the sum of square of the dimensions of the irreducible representations of a finite group is equal to the order of the group. In the present case we should have $d_{0}^{2}+d_{1}^{2}+d_{2}^{2}=6$ with $d_{k}$ the dimension of $\Gamma^{k}$. The only solution in integers of this equation is $d_{0}=d_{1}=1$ and $d_{2}=2$. Using the fact that $\chi^{k}(e)=d_{k}$, where $\chi^{k}$ is the character of $\Gamma^{k}$ and the orthogonality theorem for the characters we deduce the character table for the group $\mathrm{G}=3 m$ :

\begin{tabular}{|c|c|c|c|}
\hline & $\{e\}$ & $\mathrm{C}_{1}$ & $\mathrm{C}_{2}$ \\
\hline$\Gamma^{0}$ & 1 & 1 & 1 \\
\hline$\Gamma^{1}$ & 1 & 1 & -1 \\
\hline$\Gamma^{2}$ & 2 & -1 & 0 \\
\hline
\end{tabular}

The character $\chi_{\text {vib }}$ of $\Gamma_{\text {vib }}$ takes the following values on the classes of $\mathrm{G}=3 m: \chi_{\text {vib }}(e)=6(\mathcal{N}=4, \varphi=$ $0), \chi_{\text {vib }}\left(\mathrm{C}_{1}\right)=\chi_{\mathrm{vib}}(3)=0(\mathcal{N}=1, \varphi=\pi / 3)$ and $\chi_{\text {vib }}\left(\mathrm{C}_{2}\right)=\chi_{\text {vib }}(m)=2(\mathcal{N}=2, \varphi=\pi)$ (see fig. 3$)$. We deduce that $\left\langle\chi_{0} \mid \chi_{\text {vib }}\right\rangle=2,\left\langle\chi_{1} \mid \chi_{\text {vib }}\right\rangle=0$ and $\left\langle\chi_{2} \mid \chi_{\text {vib }}\right\rangle=2$. Accordingly,

$$
\Gamma_{\mathrm{vib}}=2 \Gamma^{0} \oplus 2 \Gamma^{2}
$$




\section{EPJ Web of Conferences}

The vibration modes will consist in 2 singlets with symmetry $\Gamma^{0}$ and two doublets with symmetry $\Gamma^{2}$. Note that $\Gamma=3 \Gamma^{0} \oplus \Gamma^{1} \oplus 4 \Gamma^{2}$. Applying the projection-shift operators $p_{n m}^{k}$ (see eq. (3.11)) on trial vectors $\vec{v}=\sum_{i \alpha} v_{i \alpha} \hat{e}_{i \alpha}$ the basis vectors of the invariant subspaces may be obtained. With $p_{00}^{0}=\frac{1}{6} \sum_{g \in \mathrm{G}} \rho(g)$ one obtains $\hat{e}_{1 \alpha}+\hat{e}_{2 \alpha}+\hat{e}_{3 \alpha}$ (from $\hat{e}_{1 \alpha}$ ) and $\hat{e}_{4 z}$ (from $\hat{e}_{4 z}$ ). Combining these to reject global motion of the molecule leads to $\sum_{i} \hat{e}_{i r_{i}}$ describing in-phase radial vibration and $\sum_{i} \hat{e}_{i z}-$ $\sum_{i=1,3}\left(m_{i} / m_{4}\right) \hat{e}_{4 z}$ describing $\pi$-phased N-H apical vibration.

\subsection{Irreducible representations of the point and space groups}

Irreducible characters and irreducible representations (up to similarity) of the point and space groups are available through tables or computer programs [6, 8-13]. It however is not useless to get a rough idea of the way they could have been determined.

Elementary algebra can suffice to get the irreducible representations (irreps) of the 32 point groups $\mathrm{G}_{\mathrm{P}}$, this by making use of a series of basic theorems, which we shall merely recall here without providing proofs:

- $\frac{1}{n_{\mathrm{G}}} \sum_{g \in \mathrm{G}} \Gamma_{j l}^{k}(g) \Gamma_{m n}^{q}\left(g^{-1}\right)=\frac{1}{d_{k}} \delta_{k q} \delta_{j n} \delta_{l m}$ (orthogonality of the irreps).

- $\frac{1}{n_{\mathrm{G}}} \sum_{g \in \mathrm{G}} \Gamma_{j l}^{k}(g) \Gamma_{n m}^{q}(g)^{\star}=\frac{1}{d_{k}} \delta_{k q} \delta_{j n} \delta_{l m}$ (orthogonality of unitary irreps).

- $\frac{1}{n_{\mathrm{G}}} \sum_{g \in \mathrm{G}}\left(\chi^{q}(g)\right)^{\star} \chi^{k}(g)=\left\langle\chi^{q} \mid \chi^{k}\right\rangle=\delta_{k q}$ (orthogonality of the irreducible characters).

- The trivial representation $\Gamma_{0}$, that trivially associates the scalar unit 1 to every group element $g$, i.e. such that $\Gamma_{0}(g)=1 \forall g \in \mathrm{G}$, always exists and is irreducible. It then follows from the orthogonality theorems that $\forall \Gamma^{k \neq 0} \sum_{g \in \mathrm{G}} \Gamma^{k}(g)_{n m}=0 \quad \& \quad \forall \chi^{k \neq 0} \sum_{g \in \mathrm{G}} \chi^{k}(g)=0$.

- If $e$ is the unit of $\mathrm{G}$ then $\chi^{k}(e)=d_{k}$ (dimension of $\Gamma^{k}$ ).

- $\sum_{k} \chi^{k}(e) \chi^{k}(g)=n_{\mathrm{G}} \delta_{e g}$ (order of $\mathrm{G}$ ), in particular $\sum_{k} d_{k}^{2}=n_{\mathrm{G}}$.

- The number of the irreducible representations (irreps) of a finite group $\mathrm{G}$ is equal to the number of its conjugacy classes $\mathrm{C}_{u}$.

- $\sum_{u} n_{u}\left(\chi_{u}^{q}\right)^{\star}\left(\chi_{u}^{k}\right)=n_{\mathrm{G}} \delta_{k q}$ (character table line by line orthogonality). $\chi_{u}^{k}$ is the value of the character of the irreps $\Gamma^{k}$ over the class $\mathrm{C}_{u}$ and $n_{u}$ the number of elements in $\mathrm{C}_{u}$.

- $\sum_{k} n_{u}\left(\chi_{v}^{k}\right)^{\star}\left(\chi_{u}^{k}\right)=n_{\mathrm{G}} \delta_{u v}$ (character table column by column orthogonality).

- The dimension $d_{k}$ of the irreps $\Gamma^{k}$ divides the order $n_{\mathrm{G}}$ of $\mathrm{G}: \frac{n_{\mathrm{G}}}{d_{k}}=$ integer. More generally, if $\mathrm{H}$ is an invariant abelian subgroup of $\mathrm{G}$ with index $[\mathrm{G}: \mathrm{H}]=\frac{n_{\mathrm{G}}}{n_{\mathrm{H}}}$ then $\frac{[\mathrm{G}: \mathrm{H}]}{d_{k}}=$ integer.

Also useful to build the irreps are the decomposition of the group into direct product of subgroups or semi-direct products of subgroups. An extremely powerful procedure is to generate representations by induction from subgroups, in particular by the so-called method of little groups when the group is the semi-direct product of a subgroup by an abelian subgroup.

The irreducible representations of the translation group $\mathrm{G}_{\mathrm{T}}$ of a crystal are easily obtained. The group is abelian so that every of its element makes up a conjugacy class by its own. It follows that the number of the irreps is equal to the order of the group and therefore all its irreps are one-dimensional. $\mathrm{G}_{\mathrm{T}}$ is the direct product $\mathrm{G}_{\mathrm{T} a} \times \mathrm{G}_{\mathrm{T} b} \times \mathrm{G}_{\mathrm{T} c}$ of the groups of translations along each of the three independent basis vectors $\vec{a}, \vec{b}, \vec{c}$ of the crystal lattice. Under the cyclic Born-von Karman conditions the group $\mathrm{G}_{\mathrm{T} a}$ (resp. $\mathrm{G}_{\mathrm{T} b}, \mathrm{G}_{\mathrm{T} c}$ ) gets cyclic, that is to say there exits integers $\mathrm{N}_{a}$ (resp. $\mathrm{N}_{b}, \mathrm{~N}_{c}$ ) such that the successive $\mathrm{N}_{a}$ (resp. $\mathrm{N}_{b}, \mathrm{~N}_{c}$ ) applications of the translation $\vec{a}$ (resp. $\vec{b}, \vec{c}$ ) gives back the unit $\overrightarrow{0}$ of $\mathrm{G}_{\mathrm{T} a}$ (resp. $\mathrm{G}_{\mathrm{T} b}, \mathrm{G}_{\mathrm{T} c}$ ). It follows that the irreps $\Delta^{k_{a}}$ of $\mathrm{G}_{\mathrm{T} a}$ are solutions of the equation $\Delta^{k_{a}}\left(\mathrm{~N}_{a} \vec{a}\right)=$ $\Delta^{k_{a}}(\vec{a}) \cdots \Delta^{k_{a}}(\vec{a})=\left[\Delta^{k_{a}}(\vec{a})\right]^{\mathrm{N}_{a}}=\Delta^{k_{a}}(\overrightarrow{0})=1$. This means that $\Delta^{k_{a}}(\vec{a})$ is an $\mathrm{N}_{a}$-th root of 1 . We may put $\Delta^{k_{a}}(\vec{a})=\exp \left(-2 i \pi \vec{k}_{a} \cdot \vec{a}\right)$ where $\vec{k}_{a}=\left(k_{a} / \mathrm{N}_{a}\right) \vec{a}^{\star}\left(k_{a}=1, \ldots, \mathrm{N}_{a}\right)$ and $\vec{a}{ }^{\star} \cdot \vec{a}=1$. Similarly the irreps of $\mathrm{G}_{\mathrm{T} b}$ and $\mathrm{G}_{\mathrm{T} c}$ may be put to $\Delta^{k_{b}}(\vec{b})=\exp \left(-2 i \pi \vec{k}_{b} \cdot \vec{b}\right)$ and $\Delta^{k_{c}}(\vec{c})=\exp \left(-2 i \pi \vec{k}_{c} \cdot \vec{c}\right)$ where $\vec{k}_{b}=\left(k_{b} / \mathrm{N}_{b}\right) \vec{b}^{\star}\left(k_{b}=1, \ldots, \mathrm{N}_{b}\right)$ and $\vec{b}^{\star} \cdot \vec{b}=1$ and $\vec{k}_{c}=\left(k_{c} / \mathrm{N}_{c}\right) \vec{c}^{\star}\left(k_{c}=1, \ldots, \mathrm{N}_{c}\right)$ and $\vec{c}^{\star} \cdot \vec{c}=1$. We easily identify the reciprocal lattice basis vectors $\vec{a}^{\star}, \vec{b}^{\star}, \vec{c}^{\star}$. Now, letting $\mathrm{N}_{a}$ (resp. $\mathrm{N}_{b}, \mathrm{~N}_{c}$ ) tend 


\section{Contribution of Symmetries in Condensed Matter}

to infinity and making use of the fact that $\mathrm{G}_{\mathrm{T}}=\mathrm{G}_{\mathrm{T} a} \times \mathrm{G}_{\mathrm{T} b} \times \mathrm{G}_{\mathrm{T} c}$, the irreps of $\mathrm{G}_{\mathrm{T}}$ are labeled by the reciprocal vectors $\vec{k}$ and are given as

$$
\Delta^{\vec{k}}(\vec{T})=\exp (-2 i \pi \vec{k} \cdot \vec{T})
$$

for every invariance by translation $\vec{T}=u \vec{a}+v \vec{b}+w \vec{c}(u, v, w$ integers) of the crystal lattice.

The irreducible representations of the space groups $\mathrm{G}$ are more technical to determine. We thus shall content ourselves with a very qualitative description. A standard procedure is first to observe that its abelian subgroup of translation $\mathrm{G}_{\mathrm{T}}$ is a normal subgroup, that is to say $\forall h \in \mathrm{G}_{\mathrm{T}} \forall g \in \mathrm{G}, g^{-1} h g \in \mathrm{G}_{\mathrm{T}}$ : if $h=\vec{T}$ (translation of vector $\vec{T}$ ) and $g=(W, w+\vec{S}$ ) (orientation symmetry W possibly combined with a fractional translation $w$ and a lattice translation $\vec{S}$ ) then $g^{-1} h g=W^{-1} \vec{T}$, which do belong to $\mathrm{G}_{\mathrm{T}}$ since the crystal lattice should be consistent with the orientation symmetry. An equivalence relation on the irreps of $\mathrm{G}_{\mathrm{T}}$ then can be defined as $\Delta^{\vec{k}} \sim \Delta^{\vec{q}} \Leftrightarrow \Delta^{\vec{k}}\left(W^{-1} \vec{T}\right)=\Delta^{\vec{q}}(\vec{T}) \Leftrightarrow W^{\star} \vec{k}=\vec{q}+\vec{\tau}$, where $W^{\star}$ means that the orientation symmetry applies in the reciprocal space $\left(\left(W^{\star} \vec{k}\right) \cdot \vec{T}=\vec{k} \cdot\left(W^{-1} \vec{T}\right)\right)$ and $\vec{\tau}=h \vec{a}^{\star}+k \vec{b}^{\star}+l \vec{c}^{\star}(h, k, l$ integers $)$ is a reciprocal lattice vector. The set $\left\{W: W^{\star} \vec{k}=\vec{k}+\vec{\tau}\right\}$ endowed with the composition law of the space group, makes up the little co-group $\mathrm{G}_{0}(\vec{k})$ of $\Delta^{\vec{k}}$ in $\mathrm{G}$. It coincides with one of the 32 point groups. Its irreps are then easily determined. The subgroup of the space group $\mathrm{G}$ defined as

$$
\mathrm{G}_{\vec{k}}=\sum_{W \in \mathrm{G}_{0}(\vec{k})}(W, w) \mathrm{G}_{\mathrm{T}}
$$

is called the little group of $\Delta^{\vec{k}}$ in $\mathrm{G}$. The $\mathrm{G}_{\vec{k}}$ maximally factors for $\Delta^{\vec{k}}$ the expansion $\mathrm{G}=(e, 0) \mathrm{G}_{\mathrm{T}}+$ $\left(W_{1}, w_{1}\right) \mathrm{G}_{\mathrm{T}}+\left(W_{2}, w_{2}\right) \mathrm{G}_{\mathrm{T}}+\cdots$ of the space group $\mathrm{G}$ over the cosets of $\mathrm{G}_{\mathrm{T}}$ :

$$
\mathrm{G}=\sum_{\Xi \notin \mathrm{G}_{0}(\vec{k})}(\Xi, \xi) \mathrm{G}_{\vec{k}}
$$

One has $\Xi^{\star} \vec{k}=\vec{q}+\vec{\tau}$ with $\vec{q} \neq \vec{k}$ : the orientation symmetry $\Xi$ generates the star $\{\vec{k}\}$ of $\vec{k}$. An irreducible matrix representation $\delta^{\vec{k} v}$ of the little group $\mathrm{G}_{\vec{k}}$ is called small (or allowed) if its subduction (or restriction) to $\mathrm{G}_{\mathrm{T}}$ leads to the irreducible matrix representation $\Delta^{\vec{k}}$ of $\mathrm{G}_{\mathrm{T}}$. The additional label $v$ distinguishes between different matrix representations associated with the same $\Delta^{\vec{k}}$. A little group is a space group so that every of its elements $g$ is the composition of a lattice translation $\vec{T}$ and of a point symmetry $W$ possibly combined with a fractional translation $w$. Since by definition $\delta^{\vec{k} v}$ subduces $\Delta^{\vec{k}}$, it follows that

$$
\delta^{\vec{k} v}(g)=\Delta^{\vec{k}}(\vec{T}) I_{d_{v}} \delta^{\vec{k} v}(W, w)=\exp (-2 i \pi \vec{k} \cdot \vec{T}) I_{d_{v}} \delta^{\vec{k} v}(W, w) \quad \forall g \in \mathrm{G}_{\vec{k}}
$$

where $I_{d_{v}}$ is the identity matrix of dimension $d_{v}$ equal to that of $\delta^{\vec{k} v}$. As to determine the zeroth block matrix $\delta^{\vec{k} v}(W, w)$ of dimension $d_{v}$ the usage is to define new matrices $\partial^{\vec{k} v}(W)$ such that

$$
\delta^{\vec{k} v}(W, w)=\exp (-2 i \pi \vec{k} \cdot \vec{w}) \partial^{\vec{k} v}(W)
$$

As from $\delta^{\vec{k} v}\left(W_{1}, w_{1}\right) \delta^{\vec{k} v}\left(W_{2}, w_{2}\right)=\exp (-2 i \pi \vec{k} \cdot \vec{S}) \delta^{\vec{k} v}\left(W_{1} W_{2}, w_{12}\right)$ with $\vec{S}=w_{1}+W_{1} w_{2}-w_{12} \in \mathrm{G}_{\mathrm{T}}$ and where $w_{12}$ is the fractional translation associated with $W_{12}=W_{1} W_{2}$ (note that $w_{12}$ has nothing to do with $w_{1} w_{2}$ ), we deduce that

$$
\partial^{\vec{k} v}\left(W_{1}\right) \partial^{\vec{k} v}\left(W_{2}\right)=\exp \left(-2 i \pi\left(W_{1}^{\star} \vec{k}-\vec{k}\right) \cdot \vec{w}_{2}\right) \partial^{\vec{k} v}\left(W_{1} W_{2}\right)
$$

If the little group is symmorphic (all the $w$ null) or if the little group is non symmorphic but $W^{\star} \vec{k}-\vec{k}=\overrightarrow{0}$ then $\partial^{\vec{k} v}\left(W_{1}\right) \partial^{\vec{k} v}\left(W_{2}\right)=\partial^{\vec{k} v}\left(W_{1} W_{2}\right)$, and $\partial^{\vec{k} v}$ is a linear matrix representation of the little co-group $\mathrm{G}_{0}(\vec{k})$. On the other hand, if the little group is non symmorphic and there exists $(W, w)$ such that $W^{\star} \vec{k}-\vec{k} \neq \overrightarrow{0}$ then $\partial^{\vec{k} v}$ is a projective matrix representation of the little co-group $\mathrm{G}_{0}(\vec{k})$ whose determination requires 


\section{EPJ Web of Conferences}

specific techniques. This is the case when $\vec{k}$ is a point of symmetry or is on a line or a plane of symmetry of the surface of the Brillouin Zone.

It may be shown that the irreducible representation of the space group $\mathrm{G}$ are induced once and only once from the irreducible representations $\delta^{\vec{k} v}$ of its little groups $\mathrm{G}_{\vec{k}}$ :

$$
\delta_{\uparrow G}^{\{\vec{k}\} v}(g)=\sum_{h \in \mathrm{G}_{\vec{k}}} \sigma(g, h) \otimes \delta^{\vec{k} v}(h)
$$

with $\sigma(g, h)_{M, L}=1$ if $g_{L}^{-1} g g_{M} \in \mathrm{G}_{\vec{k}}$ and 0 otherwise. $g_{M}$ and $g_{L}$ are the $(\Xi, \xi)$ involved in the coset expansion $\mathrm{G}=\sum_{\Xi \notin \mathrm{G}_{0}(\vec{k})}(\Xi, \xi) \mathrm{G}_{\vec{k}}$ so the irreps $\delta_{\uparrow G}^{\{\vec{k}\} v}(g)$ are labeled by the star $\{\vec{k}\}$ of $\vec{k}$.

We shall end this section by qualitatively considering back the problem of the molecule vibrations but now in a crystal of space group $G$ with the same molecule replicated at each node of the crystal. The representation space is naturally build from the representation $\mathrm{V}$ of a single molecule as a direct sum $\bigoplus_{N_{m}} V$ where $N_{m}$ is the number, in principle infinite, of molecules (lattice points) in the crystal. A linear representation $\rho$ is built on considering the effects of the elements of $\mathrm{G}$ on the atomic displacements of the atoms of all the molecules. If $\chi$ denotes its character then the orthogonality theorem for the characters displayed in eq. (3.9) tells that the matrix representative of $\rho$ expands in terms of a direct sum of $\vec{k}$-components $\Gamma^{\vec{k}}$. As a matter of fact, $\chi$ is nothing but a Fourier Series. The corresponding basis vectors as inferred from the projection-shift operators are $\vec{k}$-components of the displacements. To further proceed with the representation analysis, it then is enough to deal with each $\vec{k}$-component separately and focus the analysis only at the little groups $\mathrm{G}_{\vec{k}}$. In the case of symmorphic space groups $\mathrm{G}$ or for $W^{\star} \vec{k}-\vec{k}=\overrightarrow{0}$ we are to some extent brought back to the simpler problem of representation analysis of a Fourier component of the physical quantity in a point group $\mathrm{G}_{\mathrm{P}}$.

\section{CONCLUSION}

Orientation and translation crystal symmetries in a crystal are mutually constrained in a discretized medium and thus are limited in number and types. As an outcome the spatial symmetry of every crystal is always described by one among a finite list of 230 space groups, with the clear advantage that these and their irreducible representations can all be tabulated. Although initially anticipated, the irreducible representations of space groups (irreps) are finally not dealt with in depth. The interested reader may consult Refs. [8-11] for further details.

\section{References}

[1] International Tables For Crystallography, Volume A, Space-group symmetry (Kluwer Academic Press, 5th ed., 2002), website: http://it.iucr.org/

[2] A. Janner, Acta Cryst. A57, 378 (2001)

[3] H. Hiller, Acta Cryst. A41, 541 (1985)

[4] H. Weyl, Symmetry (Princeton University Press, 1952)

[5] M. Bürger, Elementary Crystallography (John Willey and sons, 1978)

[6] Bilbao Crystallographic Server, website: http://www.cryst.ehu.es/

[7] M. Hulin and O. Betbeder, Théorie des groupes appliquée à la physique (Les Editions de Physique, Les Ulis, 1991)

[8] Th. Kahan, Théorie des groupes en physique classique et quantique, tome 2 (Dunod, Paris ,1971)

[9] C. J. Bradley and A. P. Cracknell, The mathematical theory of symmetry in solids - Representation theory for point groups and space groups (Clarendon Press, Oxford, 1972) 


\section{Contribution of Symmetries in Condensed Matter}

[10] O. V. Kovalev, Representation of the Crystallographic Space groups, Irreducible Representations, Induced Representations and Corepresentations (Gordon and Breach Science Publishers, Amsterdam, 2nd ed., 1993)

[11] S. C. Miller and W. F. Love, Table of Irreducible Representations of Space Groups and Corepresentations of Magnetic Space Groups (Pruett Press, Boulden, Colorado, 1967)

[12] E. R. Hovestreydt, M. I. Aroyo, H. Wondratschek, J. Appl. Cryst. 25, 544 (1992)

[13] J. Rodríguez-Carvajal, FullProf, BasIreps component, website: http://www.ill.eu/sites/fullprof/ index.html

[14] E. Wigner, Group Theory (Academic Press, New York, 1959) 